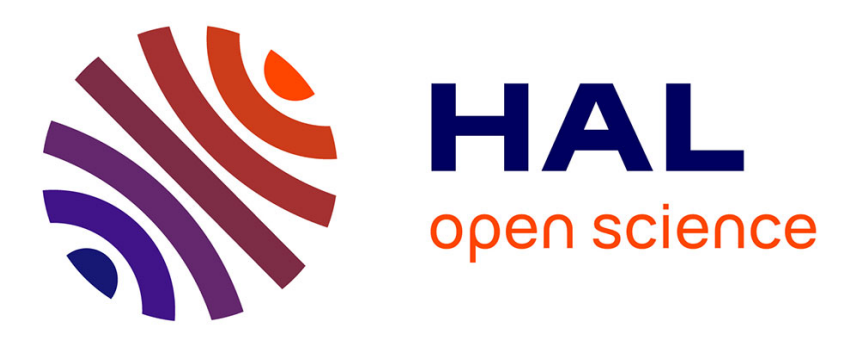

\title{
Explicit Robin-Neumann schemes for the coupling of incompressible fluids with thin-walled structures
}

\author{
Miguel Angel Fernández, Jimmy Mullaert, Marina Vidrascu
}

\section{To cite this version:}

Miguel Angel Fernández, Jimmy Mullaert, Marina Vidrascu. Explicit Robin-Neumann schemes for the coupling of incompressible fluids with thin-walled structures. Computer Methods in Applied Mechanics and Engineering, 2013, 267, pp.566-593. 10.1016/j.cma.2013.09.020 . hal-00784903v3

\section{HAL Id: hal-00784903 \\ https://hal.inria.fr/hal-00784903v3}

Submitted on 18 Sep 2013

HAL is a multi-disciplinary open access archive for the deposit and dissemination of scientific research documents, whether they are published or not. The documents may come from teaching and research institutions in France or abroad, or from public or private research centers.
L'archive ouverte pluridisciplinaire HAL, est destinée au dépôt et à la diffusion de documents scientifiques de niveau recherche, publiés ou non, émanant des établissements d'enseignement et de recherche français ou étrangers, des laboratoires publics ou privés. 
Explicit Robin-Neumann schemes for the coupling of incompressible fluids with thin-walled structures

Miguel A. Fernández, Jimmy Mullaert, Marina Vidrascu

RESEARCH

REPORT

$\mathbf{N}^{\circ} 8224$

February 2013

Project-Team Reo 



\title{
ching
}

\section{Explicit Robin-Neumann schemes for the coupling of incompressible fluids with thin-walled structures}

\author{
Miguel A. Fernández ${ }^{* \dagger}$, Jimmy Mullaert*†, Marina Vidrascu*† \\ Project-Team Reo \\ Research Report n 8224 - February 2013 - 43 pages
}

\begin{abstract}
We introduce a class of explicit coupling schemes for the numerical solution of fluid-structure interaction problems involving a viscous incompressible fluid and a general thinwalled structure (e.g., including damping and non-linear behavior). The fundamental ingredient in these methods is the (parameter free) explicit Robin interface condition for the fluid, which enables the fluid-solid splitting through appropriate extrapolations of the solid velocity and fluid stress on the interface. The resulting solution procedures are genuinely partitioned. Stability and error estimates are provided for all the variants (depending on the extrapolations), using energy arguments within a representative linear setting. In particular, we show that one of them yields added-mass free unconditional stability and optimal (first-order) time accuracy. A comprehensive numerical study, involving different examples from the literature, supports the theory.
\end{abstract}

Key-words: fluid-structure interaction, incompressible fluid, non-linear shell, visco-elasticity, time-discretization, explicit coupling scheme, loosely coupled scheme, Robin-Neumann scheme, partitioned procedure, finite element method, error estimate.

This work was supported by the French National Research Agency (ANR) through the EXIFSI project (ANR-12-JS01-0004).

* Inria, REO project-team, Rocquencourt - B.P. 105, F-78153 Le Chesnay cedex, France

$\dagger$ UPMC Univ Paris VI, REO project-team, UMR 7958 LJLL, F-75005 Paris, France

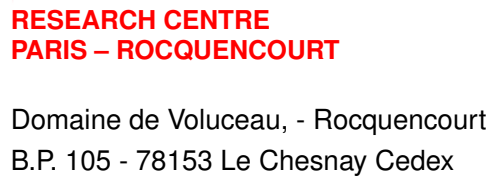




\section{Schémas Robin-Neumann explicites pour le couplage d'un fluide incompressible avec une structure mince}

Résumé : Cet article présente une famille de schémas numériques explicites qui permet de résoudre un problème d'interaction fluide-structure faisant intervenir un fluide visqueux incompressible et une structure mince (qui peut être amortie ou non linéaire). L'outil principal de ces méthodes réside dans une condition aux limites de type Robin pour le fluide (sans paramètre à ajuster), qui permet le découplage entre les deux sous-problèmes et fait intervenir une extrapolation de la vitesse du solide et des efforts du fluide. Le schéma qui en résulte est partitionné. L'article fournit des estimations d'énergie et d'erreur a priori qui permettent de montrer la stabilité et la convergence du schéma pour tous les ordres d'extrapolation lorsque le problème est linéaire. Plus précisément, on montre qu'une des variantes est à la fois inconditionnellement stable, insensible à l'effet de masse ajoutée et possède une précision en temps optimale (c'est-àdire d'ordre un). Ces résultats sont illustrés par de nombreux exemples numériques tirés de la littérature.

Mots-clés : interaction fluide-structure, fluide incompressible, coque non-linéaire, viscoélasticité, discrétisation en temps, schéma de couplage explicite, schéma faiblement couplé, schéma Robin-Neumann, algorithme partitionné, méthode des éléments finis, estimation d'erreur. 


\section{Introduction}

The mechanical interaction of a deformable thin-walled structure with an incompressible fluid flow is a widespread multi-physics problem. Its numerical simulation is of major interest in many engineering fields: from sailing boats and parachutes, to heat exchanger tubes, sloshing dynamics in tanks and the biomechanics of blood and airflow (see, e.g., [42, 54, 49, 18, 44, 35]).

A priori, the most efficient way to solve these coupled problems is to consider an explicit coupling scheme, that only involves the solution of the fluid and of the structure once per time step, through explicit interface conditions. Clearly, the resulting solution procedure is genuinely partitioned, in the sense that it facilitates the (re-)use of independent fluid and solid solvers. This is particularly convenient for the coupling with general thin-solid models, since their discretization by finite elements is known to lead to ill-conditioned matrices which require specific solvers (see, e.g., [29]).

Unfortunately, for these coupled problems, the stability of standard (Dirichlet-Neumann) explicit coupling schemes is dictated by the amount of added-mass effect in the system, irrespectively of the discretization parameters. In particular, a large fluid/solid density ratio combined with a slender and lengthy geometry gives rise to unconditional numerical instability (see, e.g., $[12,28])$. This failure explains why explicit coupling schemes have been practically ruled out for the simulation of incompressible fluid-structure interaction problems and, in part, it has motivated the tremendous amount of work devoted to improve efficiency via alternative methods, based on the more computationally onerous implicit and semi-implicit coupling paradigms (see, e.g., [41, 31, 34, 23, 17, 16, 22, 52, 3, 39, 2, 32, 30, 15, 48, 45]). Although remarkable progress has been made over the last decade, none of these approaches is able to guarantee stability and optimal accuracy by invoking the fluid and the solid solvers only once per time-step, in a genuinely partitioned fashion.

Explicit coupling schemes, circumventing the above infamous numerical instabilities, have only recently been proposed in the literature. In [8, 10], added-mass free stability is achieved through a specific Robin-Robin treatment of the interface coupling conditions, derived from Nitsche's interface method (see, e.g., [33, 46]), and a stabilization of the interface pressure fluctuations in time (weakly consistent interface compressibility). The price to pay is deterioration of the accuracy, which demands restrictive constraints on the discretization parameters (parabolicCFL condition), unless enough correction iterations are performed (see $[10,19])$.

For a linear coupled problem involving the Stokes equations and an undamped elastic thinsolid model (string or membrane), added-mass free stability and optimal (first-order) accuracy are obtained with the Robin-Neumann explicit coupling schemes introduced in [21, 20]. In short, these methods are incremental displacement-correction fractional-step schemes which introduce optimally consistent perturbations of the interface kinematic constraint. The non-incremental variant corresponds to the so-called kinematically coupled scheme, reported in [32], which is known to yield poor accuracy (see [21, 20]).

In this paper, we go further in the development of the ideas introduced in [21]. The first novelty concerns the presence of physical dissipation (damping) in the thin-solid model. It is well known that much materials exhibit viscoelastic behavior and this is, in particular, the case of biological tissue (see, e.g., [38, 11, 56]). Since viscoelastic constitutive laws (e.g., KelvinVoigt model) introduce hydrodynamic effects within the solid equation, one can be tempted to argue that its explicit coupling with the fluid is doomed to yield restrictive stability time-step restrictions and added-mass issues. This explains why, so far, solid damping has been implicitly embedded within the fluid, trough a non-standard (semi-implicit) Robin condition (see [32, 43, 7] and Remarks 2 and 3 below). Unfortunately, this strategy yields a coupling scheme which is no more explicit and, moreover, the resulting solution procedure is not partitioned, since the solid 
viscous contribution must be integrated within the fluid solver. In this work, the whole solid viscoelastic contribution is explicitly coupled with the fluid.

The second contribution of this work has to do with the formulation of the explicit coupling schemes. We propose a new Robin-Neumann coupling paradigm which enables the fluid-solid splitting exclusively in terms of the solid velocity and fluid stress on the interface. A remarkable feature of this new formulation is its intrinsic character, in the sense that it avoids the annoying extrapolations of the solid viscoelastic terms within the fluid. Hence, the resulting solution procedures are genuinely partitioned. This is, in particular, essential for the coupling with Reissner-Mindlin shell models, commonly used in engineering practice (see, e.g, [13, 4]), and that include transverse shear strain effects via rotation surface vectors (independent of the midsurface displacements). Indeed, the intrinsic Robin interface condition removes the ambiguity in the extrapolation of rotation unknowns and of non-linear terms. The explicit coupling schemes are then formulated in a fully non-linear setting, involving the incompressible Navier-Stokes equations (ALE formalism) and a non-linear viscoelastic shell model. The main idea consists in combining the Robin-Neumann splitting proposed with a explicit treatment of the fluid domain motion.

The third novelty concerns the numerical analysis of the methods within a representative linear setting, involving a Stokesian fluid and a viscoelastic thin-walled solid (string or membrane). By generalizing the arguments reported in [21], a priori energy and error estimates are provided for all the variants. The analysis shows that the sole implicit treatment of the solid inertia is enough to guarantee (added-mass free) stability. Sub-optimal time-convergence is expected for the variant without extrapolation and optimal accuracy is obtained for the schemes with firstand second-order extrapolations. In particular, the method with first-order extrapolation is (to our knowledge) the first explicit coupling scheme which guaranties unconditional stability and optimal (first-order) accuracy for the considered model problem.

The fourth contribution of this work deals with the partitioned solution of implicit coupling, via the iterative Robin-Neumann procedures introduced in [2]. So far the convergence analysis of these methods has been addressed in specific simplified models (e.g., inviscid fluid) and in particular geometrical configurations (e.g., a rectangle), using modal analysis (see [2]). In this work, we show that the the stability analysis of the explicit Robin-Neumann coupling schemes can be reshaped to derive, using energy arguments, a general result on the (added-mass free) convergence of these iterative methods. To the best of our knowledge, the error estimate proposed is the first which yields convergence towards the implicit coupling solution in such a general setting. The key argument consists in interpreting the explicit coupling schemes as single iterations of a parameter free Robin-Neumann iterative procedure for the solution of implicit coupling.

Finally, a comprehensive list of numerical experiments, based on different linear and nonlinear fluid-structure interaction examples from the literature, supports the above findings.

The paper is organized as follows. In Section 2, we present the linear continuous setting which serves as model coupled problem. In Section 3, we introduce the Robin-Neumann explicit coupling schemes and their different formulations within a linear time semi-discrete framework. We also discuss the connections with the partitioned solution of implicit coupling. Section 4 is devoted to the stability and convergence analysis of the methods. In Section 5, we present the generalization of the schemes to the non-linear case. The numerical experiments are reported in Section 6. Finally, Section 7 draws the conclusions and a few lines for further investigations. 


\section{A linear model problem}

Let $\Omega$ be a domain of $\mathbb{R}^{d}(d=2,3)$ and $\partial \Omega=\Gamma^{\mathrm{d}} \cup \Gamma^{\mathrm{n}} \cup \Sigma$ a given partition of its boundary. In what follows, the symbol $\Sigma$ stands for the fluid-structure interface. We consider a low Reynolds regime and assume that the structure undergoes infinitesimal displacements. The fluid is described by the Stokes equations in $\Omega$, and the structure is assumed to behave as a linear thin-solid (e.g., plate, membrane or shell) represented by the $(d-1)$-manifold $\Sigma$. Our coupled model problem reads therefore as follows: find the fluid velocity $\boldsymbol{u}: \Omega \times \mathbb{R}^{+} \rightarrow \mathbb{R}^{d}$, the fluid pressure $p: \Omega \times \mathbb{R}^{+} \rightarrow \mathbb{R}$, the solid displacement $\boldsymbol{d}: \Sigma \times \mathbb{R}^{+} \rightarrow \mathbb{R}^{d}$ and the solid velocity $\dot{\boldsymbol{d}}: \Sigma \times \mathbb{R}^{+} \rightarrow \mathbb{R}^{d}$ such that

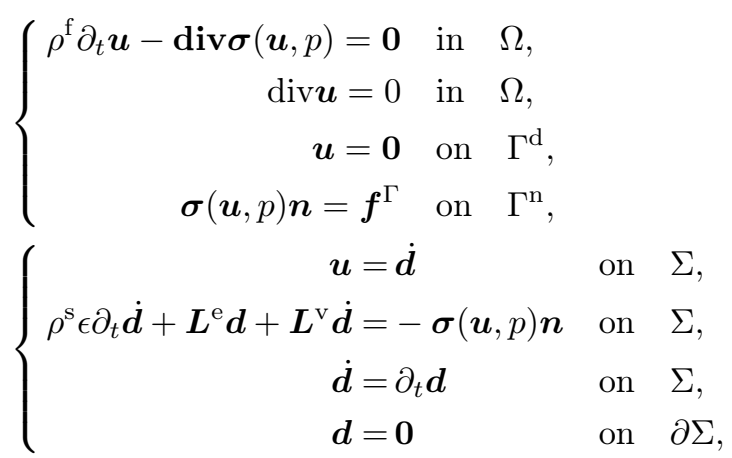

complemented with the initial conditions

$$
\boldsymbol{u}(0)=\boldsymbol{u}^{0}, \quad \boldsymbol{d}(0)=\boldsymbol{d}^{0}, \quad \dot{\boldsymbol{d}}(0)=\dot{\boldsymbol{d}}^{0} .
$$

Here, $\rho^{\mathrm{f}}$ and $\rho^{\mathrm{s}}$ respectively denote the fluid and solid densities and $\epsilon$ the solid thickness. The fluid Cauchy-stress tensor is given by

$$
\boldsymbol{\sigma}(\boldsymbol{u}, p) \stackrel{\text { def }}{=}-p \boldsymbol{I}+2 \mu \boldsymbol{\varepsilon}(\boldsymbol{u}), \quad \boldsymbol{\varepsilon}(\boldsymbol{u}) \stackrel{\text { def }}{=} \frac{1}{2}\left(\boldsymbol{\nabla} \boldsymbol{u}+\boldsymbol{\nabla} \boldsymbol{u}^{\mathrm{T}}\right),
$$

where $\mu$ stands for the fluid dynamic viscosity. The exterior unit-vector normal to $\partial \Omega$ is denoted by $\boldsymbol{n}$ and $\boldsymbol{f}^{\Gamma}$ represents a given surface force on $\Gamma^{\mathrm{n}}$. The strong formulation of the thin-solid elastic and viscous contributions are supposed to be given in terms of the surface differential operators $\boldsymbol{L}^{\mathrm{e}}$ and $\boldsymbol{L}^{\mathrm{v}}$, respectively.

The relations $(2)_{1}$ and $(2)_{2}$ enforce the kinematic and kinetic interface coupling conditions, respectively. It should be noted that the latter represents also the momentum equation of the structure. Though simplified, problem (1)-(2) features some of the main numerical issues that appear in complex nonlinear fluid-structure interaction problems involving an incompressible fluid (see, e.g., [12, 19]).

Remark 1 A widely used form of the viscous operator $\boldsymbol{L}^{\mathrm{v}}$ is given by the so-called Rayleigh damping:

$$
\boldsymbol{L}^{\mathrm{v}} \dot{\boldsymbol{d}} \stackrel{\text { def }}{=} \alpha \rho^{\mathrm{s}} \epsilon \dot{\boldsymbol{d}}+\beta \boldsymbol{L}^{\mathrm{e}} \dot{\boldsymbol{d}},
$$

where $\alpha, \beta>0$ are given parameters (see, e.g., [37]). For instance, the expression (3) is often used in artery wall modeling, where the second term corresponds to the Kelvin-Voigt model (see, e.g., $[38,11,56])$, and the first takes into account the dissipative effects of external tissue on blood vessels (see [44]). $\diamond$

Examples of thin-walled solid models entering the abstract form of $(2)_{2}$ are plate models and shell models derived under the Kirchhoff-Love kinematical assumption, the so-called membranebending shell models (see, e.g., [13, Section 4.2.3]). Richer shell models, such as the widespread 
Reissner-Mindlin shell models (see, e.g., [13, Sections 4.2.1-4.2.2]) which include transverse shear strain effects, will be considered in this work. In this case, the relations (2) have to be replaced by

$$
\left\{\begin{aligned}
\boldsymbol{u} & =\dot{\boldsymbol{d}} & & \text { on } \quad \Sigma, \\
\rho^{\mathrm{s}} \epsilon \partial_{t} \dot{\boldsymbol{d}}+\boldsymbol{L}_{\boldsymbol{d}}^{\mathrm{e}}(\boldsymbol{d}, \boldsymbol{\theta})+\boldsymbol{L}_{\boldsymbol{d}}^{\mathrm{v}}(\dot{\boldsymbol{d}}, \dot{\boldsymbol{\theta}}) & =-\boldsymbol{\sigma}(\boldsymbol{u}, p) \boldsymbol{n} & & \text { on } \quad \Sigma, \\
\boldsymbol{L}_{\boldsymbol{\theta}}^{\mathrm{e}}(\boldsymbol{d}, \boldsymbol{\theta})+\boldsymbol{L}_{\boldsymbol{\theta}}^{\mathrm{v}}(\dot{\boldsymbol{d}}, \dot{\boldsymbol{\theta}}) & =\mathbf{0} & & \text { on } \quad \Sigma, \\
\dot{\boldsymbol{d}}=\partial_{t} \boldsymbol{d}, \quad \dot{\boldsymbol{\theta}} & =\partial_{t} \boldsymbol{\theta} & & \text { on } \quad \Sigma, \\
\boldsymbol{d}=\boldsymbol{\theta} & =\mathbf{0} & & \text { on } \quad \partial \Sigma,
\end{aligned}\right.
$$

where the surface vector $\boldsymbol{\theta}: \Sigma \times \mathbb{R}^{+} \rightarrow \mathbb{R}^{3}$ (satisfying the Reissner-Mindlin kinematical assumption) describes the rotation around the normal to the mid-surface. The elastic and viscous contributions are now given in terms of the surface operators $\left(\boldsymbol{L}_{\boldsymbol{d}}^{\mathrm{e}}, \boldsymbol{L}_{\boldsymbol{\theta}}^{\mathrm{e}}\right)$ and $\left(\boldsymbol{L}_{\boldsymbol{d}}^{\mathrm{v}}, \boldsymbol{L}_{\boldsymbol{\theta}}^{\mathrm{v}}\right)$. In particular, the quasi-static relation $(4)_{3}$ represents the additional equation for the rotations.

It should be noted that the kinematic and kinetic interface coupling $(4)_{1,2}$ are enforced only in terms of the mid-surface displacement. This approach, which amounts to neglect the effect of the thickness of the shell across the interface, is a widespread modeling assumption when coupling thin-solids with three-dimensional materials (see, e.g., [14]).

For the sake of clarity, we will consider (1)-(2) as model problem for the derivation of the explicit coupling schemes in Section 3. We briefly discuss their extension to the case of the coupling with the general shell model (4) in Remark 7. The stability and convergence analysis reported in Section 4 focuses entirely on the linear coupled problem (1)-(2). In Section 6, numerical results are presented in the case of the coupling with (2), (4) and the non-linear version of (4) (see Section 5)

\section{Robin-Neumann methods}

A fundamental feature of the coupled problem (1)-(2) is its underlying Robin consistency on the interface. Indeed, by inserting $(2)_{1}$ into $(2)_{2}$ we get the expression

$$
\boldsymbol{\sigma}(\boldsymbol{u}, p) \boldsymbol{n}+\rho^{\mathrm{s}} \epsilon \partial_{t} \boldsymbol{u}=-\boldsymbol{L}^{\mathrm{e}} \boldsymbol{d}-\boldsymbol{L}^{\mathrm{v}} \dot{\boldsymbol{d}} \quad \text { on } \quad \Sigma,
$$

which yields a Robin-like interface condition for the fluid (see, e.g., [47, 32]). Instead of formulating the fluid-solid time-splitting from the original Dirichlet-Neumann coupling $(2)_{1}$ and $(2)_{2}$ (see, e.g., $[12,28]$ ), in this work we consider the Robin-Neumann interface conditions given by (5) and $(2)_{2}$. The benefits of this approach are threefold:

- the implicit treatment of the solid inertial term in (5) guarantees (added-mass free) stability;

- the explicit treatment of the solid viscoelastic terms in (5) enables the full fluid-solid splitting without compromising stability;

- the resulting schemes are genuine partitioned methods with an intrinsic explicit RobinNeumann pattern (e.g., independent of the extrapolations of the right-hand side of (5)).

\subsection{Explicit coupling schemes}

In what follows, $\tau>0$ denotes the time-step length, $t_{n} \stackrel{\text { def }}{=} n \tau$, for $n \in \mathbb{N}$, and

$$
\partial_{\tau} x^{n} \stackrel{\text { def }}{=} \frac{1}{\tau}\left(x^{n}-x^{n-1}\right),
$$


stands for the first-order backward difference. The approximation of (1)-(2) is split into two sequential sub-steps:

1. solve for the fluid with a semi-implicit time-discretization of (5), that is, the solid inertia is treated implicitly whereas the viscoelastic contribution is treated explicitly via extrapolation. This yields the following explicit Robin condition for the fluid:

$$
\boldsymbol{\sigma}\left(\boldsymbol{u}^{n}, p^{n}\right) \boldsymbol{n}+\frac{\rho^{\mathrm{s}} \epsilon}{\tau} \boldsymbol{u}^{n}=\frac{\rho^{\mathrm{s}} \epsilon}{\tau} \dot{\boldsymbol{d}}^{n-1}-\boldsymbol{L}^{\mathrm{e}} \boldsymbol{d}^{\star}-\boldsymbol{L}^{\mathrm{v}} \dot{\boldsymbol{d}}^{\star} \quad \text { on } \quad \Sigma,
$$

with

$$
\left(\boldsymbol{d}^{\star}, \dot{\boldsymbol{d}}^{\star}\right)=\left\{\begin{array}{llc}
\mathbf{0} & \text { if } & r^{\star}=0, \\
\left(\boldsymbol{d}^{n-1}, \dot{\boldsymbol{d}}^{n-1}\right) & \text { if } r^{\star}=1, \\
\left(2 \boldsymbol{d}^{n-1}-\boldsymbol{d}^{n-2}, 2 \dot{\boldsymbol{d}}^{n-1}-\dot{\boldsymbol{d}}^{n-2}\right) & \text { if } & r^{\star}=2
\end{array}\right.
$$

and where $r^{\star}$ denotes the displacement-velocity extrapolation order;

2. solve for the solid "Neumann problem" (i.e., fluid stresses are transferred to the solid):

$$
\rho^{\mathrm{s}} \epsilon \partial_{\tau} \dot{\boldsymbol{d}}^{n}+\boldsymbol{L}^{\mathrm{e}} \boldsymbol{d}^{n}+\boldsymbol{L}^{\mathrm{v}} \dot{\boldsymbol{d}}^{n}=-\boldsymbol{\sigma}\left(\boldsymbol{u}^{n}, p^{n}\right) \boldsymbol{n} \quad \text { on } \quad \Sigma .
$$

The resulting schemes enable the uncoupled sequential time-marching of the fluid and the solid (explicit coupling scheme).

Remark 2 The implicit treatment of the solid-damping, as advocated in [32, 43, 7], yields the following non-standard boundary condition for the fluid (see [20]):

$$
\boldsymbol{\sigma}\left(\boldsymbol{u}^{n}, p^{n}\right) \boldsymbol{n}+\frac{\rho^{\mathrm{s}} \epsilon}{\tau} \boldsymbol{u}^{n}+\boldsymbol{L}^{\mathrm{v}} \boldsymbol{u}^{n}=\frac{\rho^{\mathrm{s}} \epsilon}{\tau} \dot{\boldsymbol{d}}^{n-1}-\boldsymbol{L}^{\mathrm{e}} \boldsymbol{d}^{\star} \quad \text { on } \quad \Sigma .
$$

It should be noted that, in contrast to (6), the relation (9) leads to a coupling scheme which is neither explicit nor partitioned. In particular, for a simple Rayleigh modeling of the damping (see Remark 1), solving the fluid with the interface condition (9) has a computational complexity similar to the monolithic solution of a fully implicit scheme. $\diamond$

Remark 3 The interface relation (9) has clear connections with the time-stepping procedures traditionally used in the Immersed Boundary Method (see, e.g., [50, 5]), which only treat explicitly the solid elastic contributions. Another key difference with respect to (6)-(8) concerns the solid sub-step (8), which in the Immersed Boundary Method simply reduces to the displacement-velocity relation $\boldsymbol{d}^{n}=\boldsymbol{d}^{n-1}+\left.\tau \boldsymbol{u}^{n}\right|_{\Sigma}$. In other words, the structure solver is never called. The price to pay for stability is a restrictive CFL-like condition, which demands very small time-steps in practice (see, e.g., [5, Lemma 7.3] and [55]). We shall see in Section 4.4 that the combination of (6) and (8) fixes this issue. $\diamond$

In order to avoid the extrapolations, $-\boldsymbol{L}^{\mathrm{e}} \boldsymbol{d}^{\star}-\boldsymbol{L}^{\mathrm{v}} \dot{\boldsymbol{d}}^{\star}$, of the solid viscoelastic contributions in the fluid Robin-step (6), we observe that, from (8), we have

$$
\boldsymbol{L}^{\mathrm{e}} \boldsymbol{d}^{\star}+\boldsymbol{L}^{\mathrm{v}} \dot{\boldsymbol{d}}^{\star}=-\rho^{\mathrm{s}} \epsilon \partial_{\tau} \dot{\boldsymbol{d}}^{\star}-\boldsymbol{\sigma}\left(\boldsymbol{u}^{\star}, p^{\star}\right) \boldsymbol{n} \quad \text { on } \quad \Sigma
$$

for $n \geq r^{\star}+1\left(r^{\star}=1,2\right)$ and with obvious notation for $\boldsymbol{u}^{\star}$ and $p^{\star}$. Hence, by inserting (10) into (6) we get the alternative explicit Robin interface condition

$$
\boldsymbol{\sigma}\left(\boldsymbol{u}^{n}, p^{n}\right) \boldsymbol{n}+\frac{\rho^{\mathrm{s}} \epsilon}{\tau} \boldsymbol{u}^{n}=\frac{\rho^{\mathrm{s}} \epsilon}{\tau}\left(\dot{\boldsymbol{d}}^{n-1}+\tau \partial_{\tau} \dot{\boldsymbol{d}}^{\star}\right)+\boldsymbol{\sigma}\left(\boldsymbol{u}^{\star}, p^{\star}\right) \boldsymbol{n} \quad \text { on } \quad \Sigma .
$$

$\mathrm{RR} \mathrm{n}^{\circ} 8224$ 
In fact, this relation holds for $n \geq r^{\star}+1$ and for all the extrapolations $r^{\star}=0,1$ and 2 . A salient feature of this new formulation is its intrinsic character: it does not depend on the structure of the solid operator. In fact, (11) can be seen as an explicit time discretization of the following Robin condition:

$$
\boldsymbol{\sigma}(\boldsymbol{u}, p) \boldsymbol{n}+\frac{\rho^{\mathrm{s}} \epsilon}{\tau} \boldsymbol{u}=\frac{\rho^{\mathrm{s}} \epsilon}{\tau} \dot{\boldsymbol{d}}+\boldsymbol{\sigma}(\boldsymbol{u}, p) \boldsymbol{n} \quad \text { on } \quad \Sigma,
$$

in the sense that the right-hand side of (11) is an explicit approximation of the right-hand side of (12). As shown in Table 1, the extrapolation of the solid velocity has one order of accuracy higher than the resulting extrapolation of the fluid stress. This compensates the $\mathcal{O}\left(\tau^{-1}\right)$ scaling of the Robin coefficient in (12).

\begin{tabular}{|c|c|c|}
\hline$r^{\star}$ & $\dot{\boldsymbol{d}}^{n-1}+\tau \partial_{\tau} \dot{\boldsymbol{d}}^{\star}$ & $\boldsymbol{\sigma}\left(\boldsymbol{u}^{\star}, p^{\star}\right) \boldsymbol{n}$ \\
\hline 0 & $\dot{\boldsymbol{d}}^{n-1}$ & $\mathbf{0}$ \\
1 & $2 \dot{\boldsymbol{d}}^{n-1}-\dot{\boldsymbol{d}}^{n-2}$ & $\boldsymbol{\sigma}\left(\boldsymbol{u}^{n-1}, p^{n-1}\right) \boldsymbol{n}$ \\
2 & $3 \dot{\boldsymbol{d}}^{n-1}-3 \dot{\boldsymbol{d}}^{n-2}+\dot{\boldsymbol{d}}^{n-3}$ & $2 \boldsymbol{\sigma}\left(\boldsymbol{u}^{n-1}, p^{n-1}\right) \boldsymbol{n}-\boldsymbol{\sigma}\left(\boldsymbol{u}^{n-2}, p^{n-2}\right) \boldsymbol{n}$ \\
\hline
\end{tabular}

Table 1: Correspondence between the solid displacement/velocity extrapolations (7) and the resulting extrapolations of the solid velocity and the fluid stress in (11).

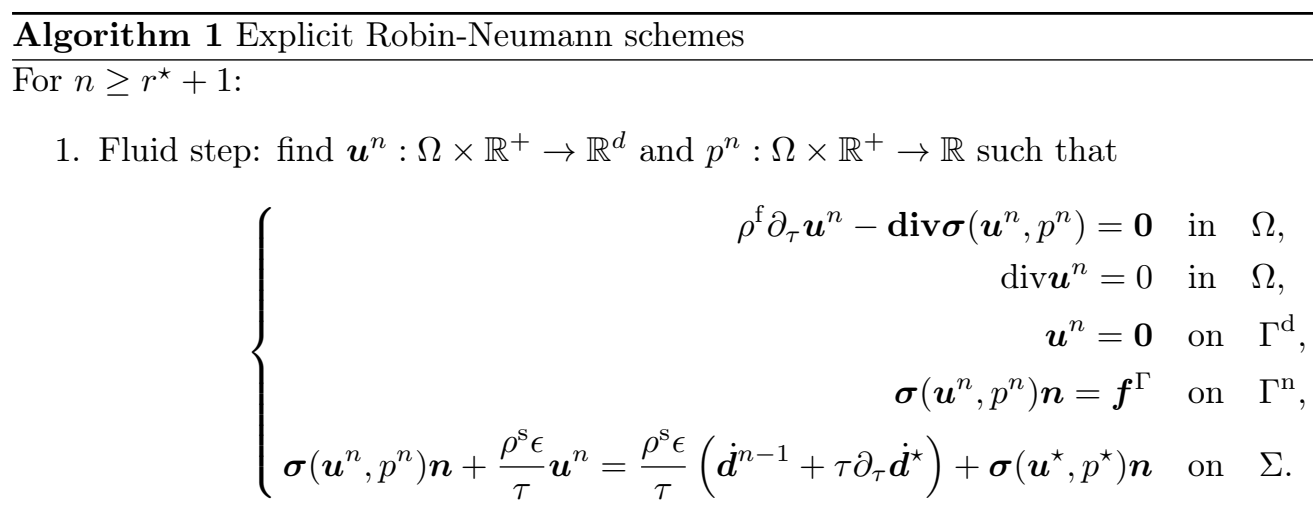

2. Solid step: find $\boldsymbol{d}^{n}: \Sigma \times \mathbb{R}^{+} \rightarrow \mathbb{R}^{d}$ and $\dot{\boldsymbol{d}}^{n}: \Sigma \times \mathbb{R}^{+} \rightarrow \mathbb{R}^{d}$ such that

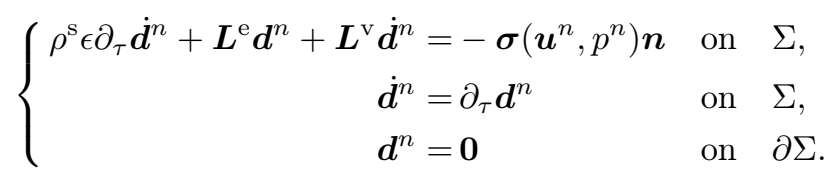

To sum up, the proposed explicit Robin-Neumann schemes are detailed in Algorithm 1, with the different extrapolations listed in Table 1.

Remark 4 It should be noted that, according to Table 1, the schemes with the first- and the second-order extrapolations $\left(r^{\star}=1,2\right)$ are multi-step methods on the interface $(n \geq 2$ and $n \geq 3$, respectively). Hence, additional data is needed to start the time-marching. In practice, this data can be obtained by performing one step of the scheme with $r^{\star}=0$, this yields $\left(\boldsymbol{u}^{1}, p^{1}, \dot{\boldsymbol{d}}^{1}\right)$, and then one step of the scheme with $r^{\star}=1$, which gives $\left(\boldsymbol{u}^{2}, p^{2}, \dot{\boldsymbol{d}}^{2}\right)$. As we shall see in Section 4 , these initializations guarantee the optimal first-order accuracy of Algorithm 1 with $r^{\star}=1,2 . \diamond$ 
Remark 5 For $r^{\star}=0$, the relations (6) and (11) coincide and, thus, the interface splittings (6)-(8) and (11)-(8) yield the same time-marching scheme for $n \geq 1$. For $r^{\star}=1$ or $r^{\star}=2$, the equivalence of the interface splittings (6)-(8) and (11)-(8) holds for $n \geq r^{\star}+1$. Hence, resulting schemes are equivalent only if they are initialized using the same procedure. $\diamond$

Remark $\mathbf{6}$ In the case of the coupling with an undamped thin-solid model (i.e., with $\boldsymbol{L}^{\mathrm{v}}=\mathbf{0}$ in $\left.(2)_{2}\right)$, Algorithm 1 yields the explicit coupling schemes introduced in [21], provided that the same initialization procedure is used. $\diamond$

Remark 7 In the case of the coupling with the general shell model (4), the interface Robin consistency (5) becomes

$$
\boldsymbol{\sigma}(\boldsymbol{u}, p) \boldsymbol{n}+\rho^{\mathrm{s}} \epsilon \partial_{t} \boldsymbol{u}=-\boldsymbol{L}_{\boldsymbol{d}}^{\mathrm{e}}(\boldsymbol{d}, \boldsymbol{\theta})-\boldsymbol{L}_{\boldsymbol{d}}^{\mathrm{v}}(\dot{\boldsymbol{d}}, \dot{\boldsymbol{\theta}}) \quad \text { on } \quad \Sigma .
$$

By applying the same argument than above, we can eliminate the extrapolations of the right-hand side in a intrinsic fashion. This yields the same interface condition (11) for the fluid. As a result, in Algorithm 1, we only need to replace the second step by

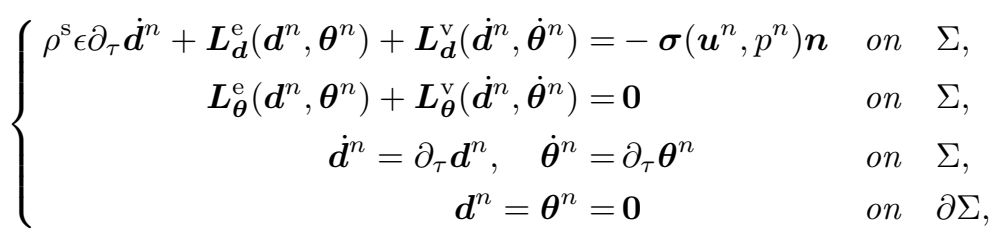

which corresponds to the implicit first-order time-discretization of the shell model $(4)_{2-5}$. This clearly demonstrates the intrinsic nature of the explicit Robin interface condition (11) and, in return, the partitioned features of Algorithm 1. $\diamond$

\subsection{Iterative partitioned solution of implicit coupling}

Algorithm 1 can be interpreted as a single iteration (with appropriate initializations) of a RobinNeumann method for the partitioned solution of the following implicit coupling scheme:

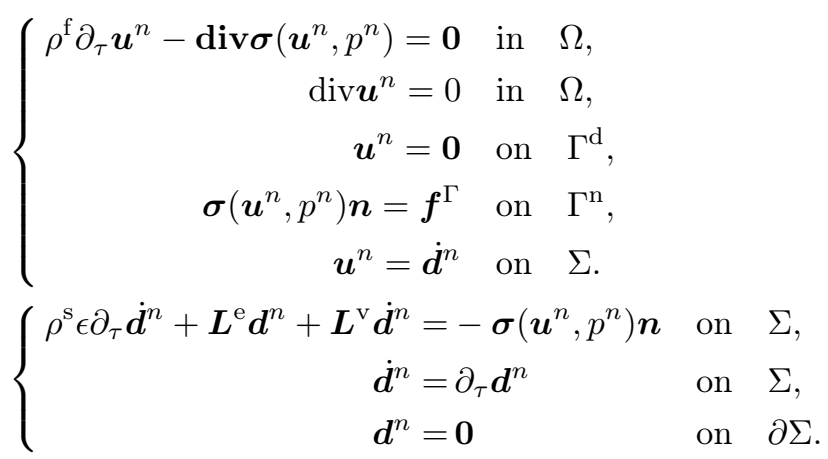

The corresponding Robin-Neumann iterations are detailed in Algorithm 2, where $\dot{\boldsymbol{d}}_{0}$ and $\boldsymbol{\sigma}\left(\boldsymbol{u}_{0}, p_{0}\right) \boldsymbol{n}$ are initialized, respectively, with $\dot{\boldsymbol{d}}^{n-1}+\tau \partial_{\tau} \dot{\boldsymbol{d}}^{\star}$ and $\boldsymbol{\sigma}\left(\boldsymbol{u}^{\star}, p^{\star}\right) \boldsymbol{n}$ for $n \geq r^{\star}+1$ (see Table 1).

This kind of iterative solution procedures has been introduced in [2], as added-mass free alternatives to the standard Dirichlet-Neumann iterations. It should be noted that the Robin coefficient $\rho^{\mathrm{s}} \epsilon / \tau$ involved in (16), has no free parameter and differs from the one originally proposed in [2]. In fact, only inertial effects are included since Algorithm 1 explicitly treats the whole viscoelastic contribution of the structure, as usual in explicit coupling schemes.

As we shall see in Section 4.5, the energy stability analysis of Algorithm 1 yields a new result on the convergence properties of Algorithm 2. 


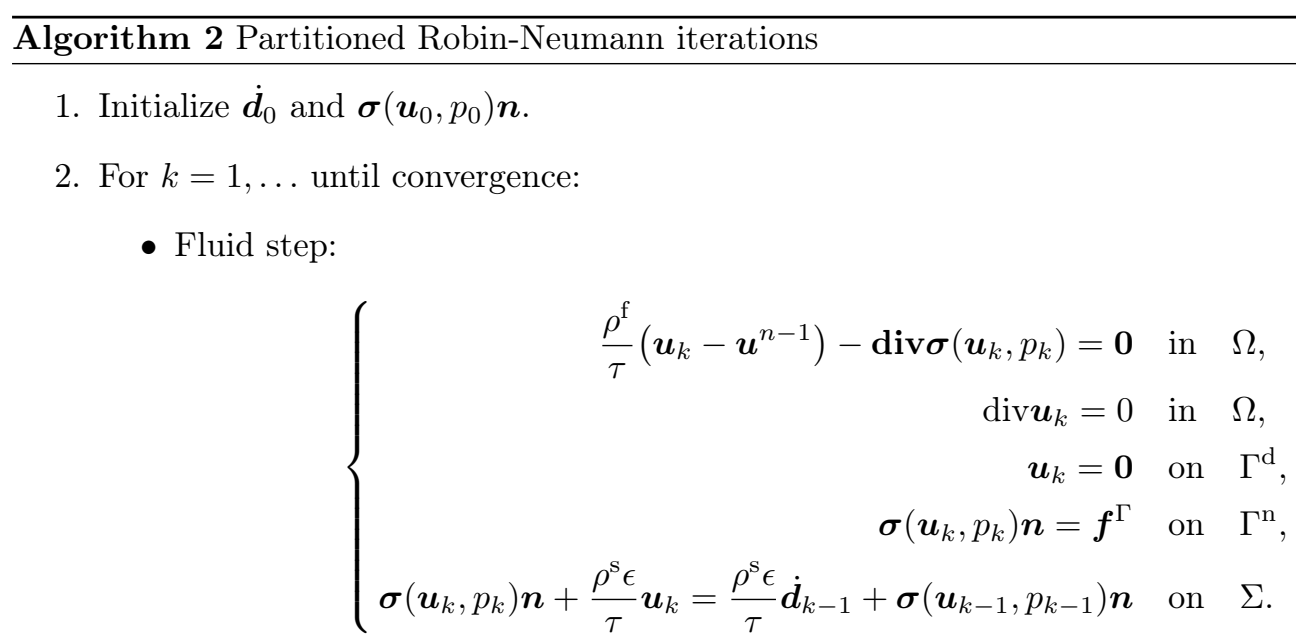

- Solid step:

$$
\left\{\begin{array}{l}
\frac{\rho^{\mathrm{s}} \epsilon}{\tau}\left(\dot{\boldsymbol{d}}_{k}-\dot{\boldsymbol{d}}^{n-1}\right)+\boldsymbol{L}^{\mathrm{e}} \boldsymbol{d}_{k}+\boldsymbol{L}^{\mathrm{v}} \dot{\boldsymbol{d}}_{k}=-\boldsymbol{\sigma}\left(\boldsymbol{u}_{k}, p_{k}\right) \boldsymbol{n} \quad \text { on } \quad \Sigma \\
\dot{\boldsymbol{d}}_{k}=\frac{1}{\tau}\left(\boldsymbol{d}_{k}-\boldsymbol{d}^{n-1}\right)
\end{array}\right.
$$

\section{Stability and convergence analysis}

This section is devoted to the stability and convergence analysis of the explicit Robin-Neumann schemes introduced in the previous section (after finite element discretization in space). In what follows, the symbols $\lesssim$ and $\gtrsim$ will indicate inequalities up to a multiplicative constant (independent of the physical and discretization parameters).

\subsection{Notation and weak formulation}

In what follows, we will consider the usual Sobolev spaces $H^{m}(\omega)(m \geq 0)$, with norm $\|\cdot\|_{m, \omega}$. The closed subspaces $H_{0}^{1}(\omega)$, of functions in $H^{1}(\omega)$ with zero trace on $\partial \omega$, and $L_{0}^{2}(\omega)$, of functions in $L^{2}(\omega)$ with zero mean in $\omega$, will also be used. The scalar product in $L^{2}(\omega)$ is denoted by $(\cdot, \cdot)_{\omega}$ and its norm by $\|\cdot\|_{0, \omega}$. In order to ease the notation, we set $(\cdot, \cdot) \stackrel{\text { def }}{=}(\cdot, \cdot)_{\Omega}$. We consider the fluid velocity and pressure functional spaces

$$
\boldsymbol{V} \stackrel{\text { def }}{=}\left\{\boldsymbol{v} \in\left[H^{1}(\Omega)\right]^{3} /\left.\boldsymbol{v}\right|_{\Gamma^{d}}=\mathbf{0}\right\}, \quad \boldsymbol{V}_{\Sigma} \stackrel{\text { def }}{=}\left\{\boldsymbol{v} \in \boldsymbol{V} /\left.\boldsymbol{v}\right|_{\Sigma}=\mathbf{0}\right\}
$$

and $Q \stackrel{\text { def }}{=} L^{2}(\Omega)$, equipped with the norms

$$
\|\boldsymbol{v}\|_{\boldsymbol{V}} \stackrel{\text { def }}{=}\left\|\mu^{\frac{1}{2}} \nabla \boldsymbol{v}\right\|_{0, \Omega}, \quad\|q\|_{Q}=\left\|\mu^{-\frac{1}{2}} q\right\|_{0, \Omega} .
$$

The space $\boldsymbol{W} \subset\left[H_{0}^{1}(\Sigma)\right]^{3}$ stands for the space of admissible displacements. At last, the following bi-linear and linear forms $a: \boldsymbol{V} \times \boldsymbol{V} \rightarrow \mathbb{R}, b: Q \times \boldsymbol{V} \rightarrow \mathbb{R}$ and $l: \boldsymbol{V} \rightarrow \mathbb{R}$, given by

$$
a(\boldsymbol{u}, \boldsymbol{v}) \stackrel{\text { def }}{=} 2 \mu(\boldsymbol{\varepsilon}(\boldsymbol{u}), \boldsymbol{\varepsilon}(\boldsymbol{v})), \quad b(q, \boldsymbol{v}) \stackrel{\text { def }}{=}-(q, \operatorname{div} \boldsymbol{v}), \quad l(v) \stackrel{\text { def }}{=}\left(\boldsymbol{f}^{\Gamma}, \boldsymbol{v}\right)_{\Gamma^{\mathrm{n}}}
$$


will be used.

The strong formulation of the thin-solid viscoelastic contributions is supposed to be given in terms of densely defined, self-adjoint and unbounded linear operators

$$
\boldsymbol{L}^{\mathrm{e}}: \boldsymbol{D}^{\mathrm{e}} \subset\left[L^{2}(\Sigma)\right]^{3} \rightarrow\left[L^{2}(\Sigma)\right]^{3}, \quad \boldsymbol{L}^{\mathrm{v}}: \boldsymbol{D}^{\mathrm{v}} \subset\left[L^{2}(\Sigma)\right]^{3} \rightarrow\left[L^{2}(\Sigma)\right]^{3},
$$

and we define two bilinear forms $a^{\mathrm{e}}$ and $a^{\mathrm{v}}$ by

$$
a^{\mathrm{e}}(\boldsymbol{d}, \boldsymbol{w})=\left(\boldsymbol{L}^{\mathrm{e}} \boldsymbol{d}, \boldsymbol{w}\right)_{\Sigma}, \quad a^{\mathrm{v}}(\dot{\boldsymbol{d}}, \boldsymbol{w})=\left(\boldsymbol{L}^{\mathrm{v}} \dot{\boldsymbol{d}}, \boldsymbol{w}\right)_{\Sigma}
$$

for all $\boldsymbol{d} \in \boldsymbol{D}^{\mathrm{e}}, \dot{\boldsymbol{d}} \in \boldsymbol{D}^{\mathrm{v}}$ and $\boldsymbol{w} \in \boldsymbol{W}$. We further assume that $a^{\mathrm{e}}$ and $a^{\mathrm{v}}$ are inner-products into $\boldsymbol{W}$ and that, endowed with the inner-product $a^{\mathrm{e}}, \boldsymbol{W}$ is a Hilbert space. We set

$$
\|\boldsymbol{w}\|_{\mathrm{e}} \stackrel{\text { def }}{=}\left(a^{\mathrm{e}}(\boldsymbol{w}, \boldsymbol{w})\right)^{\frac{1}{2}}, \quad\|\boldsymbol{w}\|_{\mathrm{v}} \stackrel{\text { def }}{=}\left(a^{\mathrm{v}}(\boldsymbol{w}, \boldsymbol{w})\right)^{\frac{1}{2}},
$$

and we assume that the following continuity estimate holds

$$
\|\boldsymbol{w}\|_{\mathrm{e}}^{2} \leq \beta^{\mathrm{e}}\|\boldsymbol{w}\|_{1, \Sigma}^{2}
$$

for all $\boldsymbol{w} \in \boldsymbol{W}$, and where $\beta^{\mathrm{e}}$ is a positive constant.

The weak form of the linear coupled problem (1)-(2) reads as follows: find $(\boldsymbol{u}(t), p(t), \boldsymbol{d}(t), \dot{\boldsymbol{d}}(t)) \in$ $\boldsymbol{V} \times Q \times \boldsymbol{W} \times \boldsymbol{W}$ such that

$$
\left\{\begin{array}{l}
\left.\boldsymbol{u}\right|_{\Sigma}=\dot{\boldsymbol{d}}, \\
\rho^{\mathrm{f}}\left(\partial_{t} \boldsymbol{u}, \boldsymbol{v}\right)+a(\boldsymbol{u}, \boldsymbol{v})+b(p, \boldsymbol{v})-b(q, \boldsymbol{u}) \\
\quad+\rho^{\mathrm{s}} \epsilon\left(\partial_{t} \dot{\boldsymbol{d}}, \boldsymbol{w}\right)_{\Sigma}+a^{\mathrm{e}}(\boldsymbol{d}, \boldsymbol{w})+a^{\mathrm{v}}(\dot{\boldsymbol{d}}, \boldsymbol{w})=l(\boldsymbol{v}), \\
\dot{\boldsymbol{d}}=\partial_{t} \boldsymbol{d}
\end{array}\right.
$$

for all $(\boldsymbol{v}, q, \boldsymbol{w}) \in \boldsymbol{V} \times Q \times \boldsymbol{W}$ with $\left.\boldsymbol{v}\right|_{\Sigma}=\boldsymbol{w}$.

\subsection{Space discretization: fully discrete schemes}

Let $\left\{\mathcal{T}_{h}\right\}_{0<h<1}$ be a family of quasi-uniform triangulations of $\Omega$. The subscript $h \in(0,1]$ refers to the level of refinement of the triangulation. In what follows, we let $X_{h}$ and $M_{h}$ denote, respectively, the standard spaces of continuous and (possibly) discontinuous piecewise polynomial functions of degree $k \geq 1$ and $l \geq 0(k-1 \leq l \leq k)$ :

$$
\begin{aligned}
& X_{h} \stackrel{\text { def }}{=}\left\{v_{h} \in C^{0}(\bar{\Omega}) / v_{h \mid K} \in \mathbb{P}_{k}(K) \quad \forall K \in \mathcal{T}_{h}\right\}, \\
& M_{h} \stackrel{\text { def }}{=}\left\{q_{h} \in Q / q_{h \mid K} \in \mathbb{P}_{l}(K) \quad \forall K \in \mathcal{T}_{h}\right\} .
\end{aligned}
$$

For the approximation of the fluid velocity we will consider the space $\boldsymbol{V}_{h} \stackrel{\text { def }}{=}\left[X_{h}\right]^{d} \cap \boldsymbol{V}$ and for the pressure we will use either $Q_{h} \stackrel{\text { def }}{=} M_{h}$ or $Q_{h} \stackrel{\text { def }}{=} M_{h} \cap C^{0}(\bar{\Omega})$. Whenever the considered velocity/pressure pair fails to satisfy the standard inf-sup condition, we consider a symmetric pressure stabilization method (see [9, 21]), given in terms of a positive and symmetric bi-linear form $s_{h}: Q_{h} \times Q_{h} \rightarrow \mathbb{R}$.

The discrete space for the solid displacement and velocity is chosen as the trace space $\boldsymbol{W}_{h} \stackrel{\text { def }}{=}$ $\left\{\left.\boldsymbol{v}_{h}\right|_{\Sigma} / \boldsymbol{v}_{h} \in \boldsymbol{V}_{h}\right\} \cap \boldsymbol{W}$. Hence, the fluid and solid space discretizations match at the interface. At last, we introduce the standard fluid-sided discrete lifting operator $\mathcal{L}_{h}: \boldsymbol{W}_{h} \rightarrow \boldsymbol{V}_{h}$, such that, for all $\boldsymbol{w}_{h} \in \boldsymbol{W}_{h},\left.\left(\mathcal{L}_{h} \boldsymbol{w}_{h}\right)\right|_{\Sigma}=\boldsymbol{w}_{h}$ and $\mathcal{L}_{h} \boldsymbol{w}_{h}$ vanishes in all nodal values whose support is out of the interface.

After finite element discretization in space, the fully discrete version of Algorithm 1 is detailed in Algorithm 3. In particular, it is worth noting that fluid stresses on the interface are evaluated in a consistent fashion, as variational residuals, in terms of the discrete lifting operator $\mathcal{L}_{h}$. 


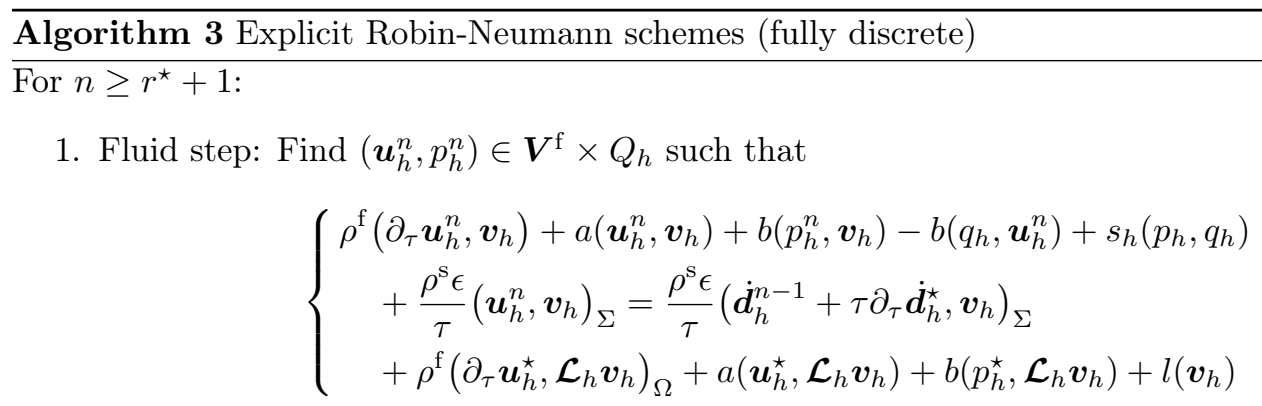

for all $\left(\boldsymbol{v}_{h}, q_{h}\right) \in \boldsymbol{V}_{h} \times Q_{h}$ with $\left.\boldsymbol{v}_{h}\right|_{\Sigma} \in \boldsymbol{W}_{h}$.

2. Solid step: Find $\left(\dot{\boldsymbol{d}}_{h}^{n}, \boldsymbol{d}_{h}^{n}\right) \in \boldsymbol{W}_{h} \times \boldsymbol{W}_{h}$, such that

$$
\left\{\begin{array}{l}
\dot{\boldsymbol{d}}_{h}^{n}=\partial_{\tau} \boldsymbol{d}_{h}^{n} \\
\rho^{\mathrm{s}} \epsilon\left(\partial_{\tau} \dot{\boldsymbol{d}}_{h}^{n}, \boldsymbol{w}_{h}\right)_{\Sigma}+a^{\mathrm{e}}\left(\boldsymbol{d}_{h}^{n}, \boldsymbol{w}_{h}\right)+a^{\mathrm{v}}\left(\dot{\boldsymbol{d}}_{h}^{n}, \boldsymbol{w}_{h}\right) \\
\quad=-\rho^{\mathrm{f}}\left(\partial_{\tau} \boldsymbol{u}_{h}^{n}, \mathcal{L}_{h} \boldsymbol{w}_{h}\right)-a\left(\boldsymbol{u}_{h}^{n}, \mathcal{L}_{h} \boldsymbol{w}_{h}\right)-b\left(p_{h}^{n}, \mathcal{L}_{h} \boldsymbol{w}_{h}\right)
\end{array}\right.
$$

for all $\boldsymbol{w}_{h} \in \boldsymbol{W}_{h}$.

\subsection{Kinematic perturbation of implicit coupling}

In what follows, we shall make use of discrete reconstructions, $\boldsymbol{L}_{h}^{\mathrm{e}}: \boldsymbol{W} \rightarrow \boldsymbol{W}_{h}$ and $\boldsymbol{L}_{h}^{\mathrm{v}}: \boldsymbol{W} \rightarrow$ $\boldsymbol{W}_{h}$, of the elastic and viscous solid operators, defined by the relations

$$
\left(\boldsymbol{L}_{h}^{\mathrm{e}} \boldsymbol{w}, \boldsymbol{w}_{h}\right)_{\Sigma}=a^{\mathrm{e}}\left(\boldsymbol{w}, \boldsymbol{w}_{h}\right), \quad\left(\boldsymbol{L}_{h}^{\mathrm{v}} \boldsymbol{w}, \boldsymbol{w}_{h}\right)_{\Sigma}=a^{\mathrm{v}}\left(\boldsymbol{w}, \boldsymbol{w}_{h}\right)
$$

for all $\left(\boldsymbol{w}, \boldsymbol{w}_{h}\right) \in \boldsymbol{W} \times \boldsymbol{W}_{h}$.

An important feature of Algorithm 3 is that it can be interpreted as kinematic perturbations of an underlying implicit coupling scheme. Indeed, replacing $n$ by $\star$ in $(21)_{2}$ with $\boldsymbol{w}_{h}=\left.\boldsymbol{v}_{h}\right|_{\Sigma}$ and adding the resulting expression to (20) yields

$$
\begin{aligned}
\rho^{\mathrm{f}}\left(\partial_{\tau} \boldsymbol{u}_{h}^{n}, \boldsymbol{v}_{h}\right)+a\left(\boldsymbol{u}_{h}^{n}, \boldsymbol{v}_{h}\right)+b\left(p_{h}^{n}, \boldsymbol{v}_{h}\right)-b\left(q_{h}, \boldsymbol{u}_{h}^{n}\right)+s_{h}\left(p_{h}, q_{h}\right) \\
\quad+\frac{\rho^{\mathrm{s}} \epsilon}{\tau}\left(\boldsymbol{u}_{h}^{n}, \boldsymbol{v}_{h}\right)_{\Sigma}=\frac{\rho^{\mathrm{s}} \epsilon}{\tau}\left(\dot{\boldsymbol{d}}_{h}^{n-1}, \boldsymbol{v}_{h}\right)_{\Sigma}-a^{\mathrm{e}}\left(\boldsymbol{d}_{h}^{\star}, \boldsymbol{v}_{h}\right)-a^{\mathrm{v}}\left(\dot{\boldsymbol{d}}_{h}^{\star}, \boldsymbol{v}_{h}\right)+l\left(\boldsymbol{v}_{h}\right)
\end{aligned}
$$

for all $\left(\boldsymbol{v}_{h}, q_{h}\right) \in \boldsymbol{V}_{h} \times Q_{h}$ with $\left.\boldsymbol{v}_{h}\right|_{\Sigma} \in \boldsymbol{W}_{h}$ and $n \geq r^{\star}+1$. Hence, by taking $\left(\boldsymbol{v}_{h}, q_{h}\right)=\left(\mathcal{L}_{h} \boldsymbol{w}_{h}, 0\right)$ in (23), subtracting the resulting expression from $(21)_{2}$ and since $l\left(\mathcal{L}_{h} \boldsymbol{w}_{h}\right)=0$, we obtain

$$
\frac{\rho^{\mathrm{s}} \epsilon}{\tau}\left(\dot{\boldsymbol{d}}_{h}^{n}-\boldsymbol{u}_{h}^{n}, \boldsymbol{w}_{h}\right)_{\Sigma}+a^{\mathrm{e}}\left(\boldsymbol{d}_{h}^{n}-\boldsymbol{d}_{h}^{\star}, \boldsymbol{w}_{h}\right)+a^{\mathrm{v}}\left(\dot{\boldsymbol{d}}_{h}^{n}-\dot{\boldsymbol{d}}_{h}^{\star}, \boldsymbol{w}_{h}\right)=0
$$

for all $\boldsymbol{w}_{h} \in \boldsymbol{W}_{h}$ and $n \geq r^{\star}+1$. Hence, owing to (22), we get

$$
\left.\boldsymbol{u}_{h}^{n}\right|_{\Sigma}=\dot{\boldsymbol{d}}_{h}^{n}+\frac{\tau}{\rho^{\mathrm{s}} \epsilon}\left(\boldsymbol{L}_{h}^{\mathrm{e}}\left(\boldsymbol{d}_{h}^{n}-\boldsymbol{d}_{h}^{\star}\right)+\boldsymbol{L}_{h}^{\mathrm{v}}\left(\dot{\boldsymbol{d}}_{h}^{n}-\dot{\boldsymbol{d}}_{h}^{\star}\right)\right)
$$

for $n \geq r^{\star}+1$. In addition, taking $\left.\boldsymbol{v}_{h}\right|_{\Sigma}=\boldsymbol{w}_{h}$ in (23) and adding the resulting expression to (24) yields

$$
\begin{aligned}
& \rho^{\mathrm{f}}\left(\partial_{\tau} \boldsymbol{u}_{h}^{n}, \boldsymbol{v}_{h}\right)+a\left(\boldsymbol{u}_{h}^{n}, \boldsymbol{v}_{h}\right)+b\left(p_{h}^{n}, \boldsymbol{v}_{h}\right)-b\left(q_{h}, \boldsymbol{u}_{h}^{n}\right)+s_{h}\left(p_{h}^{n}, q_{h}\right) \\
& \quad+\rho^{\mathrm{s}} \epsilon\left(\partial_{\tau} \dot{\boldsymbol{d}}_{h}^{n}, \boldsymbol{w}_{h}\right)_{\Sigma}+a^{\mathrm{e}}\left(\boldsymbol{d}_{h}^{n}, \boldsymbol{w}_{h}\right)+a^{\mathrm{v}}\left(\dot{\boldsymbol{d}}_{h}^{n}, \boldsymbol{w}_{h}\right)=l\left(\boldsymbol{v}_{h}\right)
\end{aligned}
$$


for all $\left(\boldsymbol{v}_{h}, q_{h}, \boldsymbol{w}_{h}\right) \in \boldsymbol{V}_{h} \times Q_{h} \times \boldsymbol{W}_{h}$ with $\left.\boldsymbol{v}_{h}\right|_{\Sigma}=\boldsymbol{w}_{h}$ and $n \geq r^{\star}+1$.

In summary, the relations (25) and (26) are nothing but an implicit time-discretization of (19) with the perturbed kinematic constraint (25). Therefore, in order to asses the stability and accuracy of Algorithm 3, we only need to investigate how this kinematic perturbation affects the stability and accuracy of the underlying implicit coupling scheme. This will be the topic of Sections 4.4 and 4.6 below.

\subsection{A priori energy estimates}

We define the total discrete energy, $E_{h}^{n}$, and dissipation, $D_{h}^{n}$, at time-step $t_{n}$ by

$$
\begin{aligned}
& E_{h}^{n} \stackrel{\text { def }}{=} \rho^{\mathrm{f}}\left\|\boldsymbol{u}_{h}^{n}\right\|_{0}^{2}+\rho^{\mathrm{s}} \epsilon \dot{\boldsymbol{d}}_{h}^{n}\left\|_{0, \Sigma}^{2}+\right\| \boldsymbol{d}_{h}^{n} \|_{\mathrm{e}}^{2}, \\
& \begin{array}{l}
D_{h}^{n} \stackrel{\text { def }}{=} \rho^{\mathrm{f}}\left\|\boldsymbol{u}_{h}^{n}-\boldsymbol{u}_{h}^{n-1}\right\|_{0}^{2}+\tau\left\|\boldsymbol{u}_{h}^{n}\right\|_{\boldsymbol{V}}^{2}+\tau\left|p_{h}^{n}\right|_{s_{h}}^{2}+\rho^{\mathrm{s}} \epsilon\left\|\dot{\boldsymbol{d}}_{h}^{n}-\dot{\boldsymbol{d}}_{h}^{n-1}\right\|_{0, \Sigma}^{2} \\
\quad+\left\|\boldsymbol{d}_{h}^{n}-\boldsymbol{d}_{h}^{n-1}\right\|_{\mathrm{e}}^{2}+\tau\left\|\dot{\boldsymbol{d}}_{h}^{n}\right\|_{\mathrm{v}}^{2},
\end{array}
\end{aligned}
$$

where $\left|p_{h}^{n}\right|_{s_{h}} \stackrel{\text { def }}{=}\left(s_{h}\left(p_{h}^{n}, p_{h}^{n}\right)\right)^{\frac{1}{2}}$. The following result states the energy stability of the explicit Robin-Neumann schemes given by Algorithm 3.

Theorem 1 Assume that $\boldsymbol{f}^{\Gamma}=\mathbf{0}$ (free system) and let $\left\{\left(\boldsymbol{u}_{h}^{n}, p_{h}^{n}, \boldsymbol{d}_{h}^{n}, \dot{\boldsymbol{d}}_{h}^{n}\right)\right\}_{n \geq r^{\star}+1}$ be the sequence given by Algorithm 3. The initialization procedure of Remark 4 is considered for the schemes with extrapolation $\left(r^{\star} \geq 1\right)$. The following a priori energy estimates hold:

- Without extrapolation $\left(r^{\star}=0\right)$ or with first-order extrapolation $\left(r^{\star}=1\right)$ :

$$
E_{h}^{n}+\sum_{m=r^{\star}+1}^{n} D_{h}^{m} \lesssim E_{h}^{0}
$$

for $n \geq r^{\star}+1$

- Second-order extrapolation $\left(r^{\star}=2\right)$ :

$$
E_{h}^{n}+\sum_{m=3}^{n} D_{h}^{m} \lesssim \exp \left(\frac{t_{n} \gamma}{1-\tau \gamma}\right) E_{h}^{0}
$$

for $n \geq 3$, provided that (3) and the following conditions hold

$$
\left\{\begin{aligned}
\alpha^{2} \tau^{2}+\beta^{2} \tau^{2} \frac{\left(\omega^{\mathrm{e}}\right)^{4}}{h^{4}} & \leq \frac{\delta}{4}, \\
\frac{\tau^{5}\left(\omega^{\mathrm{e}}\right)^{6}}{h^{6}}+2 \alpha \frac{\tau^{2}\left(\omega^{\mathrm{e}}\right)^{2}}{h^{2}}+2 \beta \tau^{2} \frac{\left(\omega^{\mathrm{e}}\right)^{4}}{h^{4}} & \leq \frac{\gamma}{2} \\
\tau \gamma & <1,
\end{aligned}\right.
$$

where $\omega^{\mathrm{e}} \stackrel{\text { def }}{=} C_{\mathrm{inv}} \sqrt{\beta^{\mathrm{e}} /\left(\rho^{\mathrm{s}} \epsilon\right)}, C_{\mathrm{inv}}$ denotes the constant of an inverse estimate, $0 \leq \delta \leq 1$ and $\gamma>0$.

Proof 1 See A.

Theorem 1 yields the unconditionally energy stability of the proposed explicit Robin-Neumann schemes without extrapolations and with the first-order extrapolations. Moreover, the energy 
estimates (27) are derived without any major assumption on the solid damping operator $\boldsymbol{L}^{\mathbf{v}}$, only symmetric and positiveness are required for the corresponding bi-linear form (18). In the case of a Rayleigh modeling of the solid damping (3), the variant with second-order extrapolations is energy stable, provided that the CFL-like conditions (29) are satisfied.

It is worth noting that all these variants are energy stable, irrespectively of the amount of added-mass effect in the system. This demonstrates that the implicit treatment of the solid inertial term in (5) is enough to guarantee added-mass free stability.

Remark 8 Theorem 1 generalizes the stability results reported in [21, Theorem 1] to the case $\boldsymbol{L}^{\mathrm{v}} \neq 0$. In particular, for $r^{\star}=2$ and in the case of the coupling with an undamped thin-solid model (i.e., $\boldsymbol{L}^{\mathrm{v}}=\mathbf{0}$ ) the relations (29) reduce to the $6 / 5-C F L$ condition $\tau\left(\omega^{\mathrm{e}}\right)^{\frac{6}{5}} \lesssim \gamma h^{\frac{6}{5}}$ derived in [21]. $\diamond$

Remark 9 The nature of the stability condition (29) depends on the Rayleigh coefficient $\beta$ involved in (3). For $\beta=0$, the relations (29) are fulfilled under a $6 / 5$-CFL condition $\tau=\mathcal{O}\left(h^{\frac{6}{5}}\right)$, while a parabolic-CFL constraint $\tau=\mathcal{O}\left(h^{2}\right)$ is required for $\beta>0$. $\diamond$

\subsection{Convergence of the iterative solution procedure}

A salient feature the arguments involved in the energy based stability analysis of Section 4.4 is that they can be reshaped to prove the convergence of Algorithm 2 towards the implicit coupling solution (14)-(15). This result is stated in the next theorem where, for the sake of simplicity, we have considered only the time semi-discrete version of the methods.

Theorem 2 For $n \geq 1$, let $\left(\boldsymbol{u}^{n}, p^{n}, \dot{\boldsymbol{d}}^{n}, \boldsymbol{d}^{n}\right)$ be given by the implicit scheme (14)-(15) and $\left\{\left(\boldsymbol{u}_{k}, p_{k}, \dot{\boldsymbol{d}}_{k}, \boldsymbol{d}_{k}\right)\right\}_{k \geq 1}$ be the sequence of approximations given by Algorithm 2. Then, the following estimate holds

$$
\begin{aligned}
\sum_{k=1}^{\infty}\left(\rho^{\mathrm{f}}\left\|\boldsymbol{u}_{k}-\boldsymbol{u}^{n}\right\|_{0, \Omega}^{2}+\rho^{\mathrm{s}} \epsilon\left\|\dot{\boldsymbol{d}}_{k}-\dot{\boldsymbol{d}}^{n}\right\|_{0, \Sigma}^{2}+\left\|\boldsymbol{d}_{k}-\boldsymbol{d}^{n}\right\|_{\mathrm{e}}^{2}\right) & \\
& \lesssim\left\|\boldsymbol{d}_{0}-\boldsymbol{d}^{n}\right\|_{\mathrm{e}}^{2}+\tau\left\|\dot{\boldsymbol{d}}_{0}-\dot{\boldsymbol{d}}^{n}\right\|_{\mathrm{v}}^{2}+\frac{\tau^{2}}{\rho^{\mathrm{s}} \epsilon}\left\|\boldsymbol{L}^{\mathrm{e}}\left(\boldsymbol{d}_{0}-\boldsymbol{d}^{n}\right)+\boldsymbol{L}^{\mathrm{v}}\left(\dot{\boldsymbol{d}}_{0}-\dot{\boldsymbol{d}}^{n}\right)\right\|_{0, \Sigma}^{2} .
\end{aligned}
$$

In particular, we have

$$
\lim _{k \rightarrow \infty}\left(\left\|\boldsymbol{u}_{k}-\boldsymbol{u}^{n}\right\|_{0, \Omega}+\left\|\dot{\boldsymbol{d}}_{k}-\dot{\boldsymbol{d}}^{n}\right\|_{0, \Sigma}+\left\|\boldsymbol{d}_{k}-\boldsymbol{d}^{n}\right\|_{\mathrm{e}}\right)=0
$$

Proof 2 See $B$.

To the best of our knowledge, the result stated in Theorem 2 is the first which guarantees the convergence of a Robin-Neumann iterative procedure towards the implicit coupling solution (14)-(15). A second valuable consequence of Theorem 2 is that the pure inertial character of the Robin coefficient $\rho^{\mathrm{S}} \epsilon / \tau$ is enough to guarantee the convergence of the iterations.

\subsection{A priori error estimates}

In what follows, we shall make use of the Riesz projectors $\boldsymbol{\pi}_{h}^{\mathrm{e}}: \boldsymbol{W} \rightarrow \boldsymbol{W}_{h}$ and $\boldsymbol{\pi}_{h}^{\mathrm{v}}: \boldsymbol{W} \rightarrow \boldsymbol{W}_{h}$ associated to the inner-products $a^{\mathrm{e}}$ and $a^{\mathrm{v}}$ given by (18). Thus, for all $\boldsymbol{w} \in \boldsymbol{W}, \boldsymbol{\pi}_{h}^{\mathrm{e}} \boldsymbol{w} \in \boldsymbol{W}_{h}$ and $\boldsymbol{\pi}_{h}^{\mathrm{v}} \boldsymbol{w} \in \boldsymbol{W}_{h}$ are respectively defined by the relations

$$
a^{\mathrm{e}}\left(\boldsymbol{w}-\boldsymbol{\pi}_{h}^{\mathrm{e}} \boldsymbol{w}, \boldsymbol{w}_{h}\right)=0, \quad a^{\mathrm{v}}\left(\boldsymbol{w}-\boldsymbol{\pi}_{h}^{\mathrm{v}} \boldsymbol{w}, \boldsymbol{w}_{h}\right)=0
$$


for all $\boldsymbol{w}_{h} \in \boldsymbol{W}_{h}$. We assume that the following error estimates hold

$$
\begin{aligned}
\left\|\boldsymbol{w}-\boldsymbol{\pi}_{h}^{\mathrm{e}} \boldsymbol{w}\right\|_{\mathrm{e}} & \lesssim h^{k}\left(\beta^{\mathrm{e}}\right)^{\frac{1}{2}}\|\boldsymbol{w}\|_{k+1, \Sigma}, \\
\left\|\boldsymbol{w}-\boldsymbol{\pi}_{h}^{\mathrm{v}} \boldsymbol{w}\right\|_{0}+h\left\|\boldsymbol{w}-\boldsymbol{\pi}_{h}^{\mathrm{v}} \boldsymbol{w}\right\|_{1, \Sigma} & \lesssim h^{k+1}\|\boldsymbol{w}\|_{k+1, \Sigma}
\end{aligned}
$$

for all $\boldsymbol{w} \in\left[H^{k+1}(\Sigma)\right]^{3} \cap \boldsymbol{W}$. For the fluid velocity, we consider the Stokes-like operator $\left(\boldsymbol{P}_{h}, R_{h}\right)$ : $\boldsymbol{V} \rightarrow \boldsymbol{V}_{h} \times Q_{h}$ defined, for all $\boldsymbol{v} \in \boldsymbol{V}$, by

$$
\left\{\begin{array}{l}
\left.\boldsymbol{P}_{h} \boldsymbol{v}\right|_{\Sigma}=\boldsymbol{\pi}_{h}^{\mathrm{v}}\left(\left.\boldsymbol{v}\right|_{\Sigma}\right) \\
a\left(\boldsymbol{P}_{h} \boldsymbol{v}, \boldsymbol{v}_{h}\right)+b\left(R_{h} \boldsymbol{v}, \boldsymbol{v}_{h}\right)=a\left(\boldsymbol{v}, \boldsymbol{v}_{h}\right) \quad \forall \boldsymbol{v}_{h} \in \boldsymbol{V}_{\Sigma, h} \\
b\left(q_{h}, \boldsymbol{P}_{h} \boldsymbol{v}\right)=s_{h}\left(q_{h}, R_{h} \boldsymbol{v}\right) \quad \forall q_{h} \in Q_{h}
\end{array}\right.
$$

We assume that the following approximation properties hold for $\boldsymbol{P}_{h}$ (see [21, Lemma 3]):

$$
\begin{aligned}
& \left\|\boldsymbol{v}-\boldsymbol{P}_{h} \boldsymbol{v}\right\|_{\boldsymbol{V}}+\left|R_{h} \boldsymbol{v}\right|_{s_{h}} \lesssim \mu^{\frac{1}{2}} h^{k}\|\boldsymbol{v}\|_{k+1, \Omega} \\
& \left\|\boldsymbol{v}-\boldsymbol{P}_{h} \boldsymbol{v}\right\|_{0, \Omega} \lesssim h^{k+1}\left(c_{\mu} \mu^{\frac{1}{2}}\|\boldsymbol{v}\|_{k+1, \Omega}+\tilde{c}_{\mu}\|\boldsymbol{v}\|_{k+1, \Sigma}\right)
\end{aligned}
$$

for all $\boldsymbol{v} \in\left[H^{k+1}(\Omega)\right]^{d}$ with $\left.\boldsymbol{v}\right|_{\Sigma} \in\left[H^{k+1}(\Sigma)\right]^{d}$ and $\operatorname{div} \boldsymbol{v}=0$. The symbols $c_{\mu}$ and $\tilde{c}_{\mu}$ denote positive constants depending only on $\Omega$ and $\mu$.

Remark 10 The estimates (33) hold for inf-sup compatible velocity/pressure approximations (with $\left.s_{h}=0\right)$ or under suitable assumptions on the symmetric stabilization operator $s_{h}$ (see [21, Section 3.1.1]). In particular, the consistency of $s_{h}$ is said to be of order $\tilde{l} \in\{l, l+1\}$ if there exits a projection operator $\Pi_{h}: Q \rightarrow Q_{h}$ such that

$$
\begin{aligned}
\left|\Pi_{h} q\right|_{s_{h}} & \lesssim \mu^{-\frac{1}{2}} h^{\tilde{l}}\|q\|_{\tilde{l}, \Omega} \quad \forall q \in H^{\tilde{l}}(\Omega), \\
\left\|q-\Pi_{h} q\right\|_{Q} & \lesssim \mu^{-\frac{1}{2}} h^{l+1}\|q\|_{l+1, \Omega} \quad \forall q \in H^{l+1}(\Omega) .
\end{aligned}
$$

Examples of such stabilization operators are discussed in [9]. $\diamond$

We assume that the exact solution of problem (19) has the following regularity, for a given final time $T \geq \tau$ :

$$
\begin{aligned}
\boldsymbol{u} & \in\left[H^{1}\left(0, T ; H^{k+1}(\Omega)\right)\right]^{d},\left.\quad \boldsymbol{u}\right|_{\Sigma} \in\left[H^{1}\left(0, T ; H^{k+1}(\Sigma)\right)\right]^{d}, \\
\partial_{t t} \boldsymbol{u} & \in\left[L^{2}\left(0, T ; L^{2}(\Omega)\right)\right]^{d},\left.\quad \partial_{t t} \boldsymbol{u}\right|_{\Sigma} \in\left[L^{2}\left(0, T ; L^{2}(\Sigma)\right)\right]^{d}, \\
p & \in C^{0}\left([0, T] ; H^{\tilde{l}}(\Omega)\right)
\end{aligned}
$$

and

$$
\boldsymbol{L}^{\mathrm{e}} \boldsymbol{d}+\boldsymbol{L}^{\mathrm{v}} \dot{\boldsymbol{d}} \in\left\{\begin{array}{llc}
{\left[C^{0}\left([0, T] L^{2}(\Sigma)\right)\right]^{d}} & \text { if } & r^{\star}=0, \\
{\left[H^{1}\left(0, T ; L^{2}(\Sigma)\right)\right]^{d}} & \text { if } \quad r^{\star}=1, \\
{\left[H^{2}\left(0, T ; L^{2}(\Sigma)\right)\right]^{d}} & \text { if } \quad r^{\star}=2 .
\end{array}\right.
$$

In (35), the symbol $\tilde{l} \in\{l, l+1\}$ denotes the consistency order of $s_{h}$ (see Remark 10). We take $\tilde{l}=l$ in the case of inf-sup compatible velocity/pressure approximations.

At last, we define the energy-norm of the error, at the time step $t_{n}$, as

$$
\begin{aligned}
\mathcal{E}_{h}^{n} \stackrel{\text { def }}{=}\left(\rho^{\mathrm{f}}\right)^{\frac{1}{2}}\left\|\boldsymbol{u}\left(t_{n}\right)-\boldsymbol{u}_{h}^{n}\right\|_{0, \Omega}+\left(\sum_{m=r^{\star}+1}^{n} \tau\left\|\boldsymbol{u}\left(t_{m}\right)-\boldsymbol{u}_{h}^{m}\right\|_{\boldsymbol{V}}^{2}\right)^{\frac{1}{2}}+\left(\sum_{m=r^{\star}+1}^{n} \tau\left|p_{h}^{m}\right|_{s_{h}}^{2}\right)^{\frac{1}{2}} & \\
& +\left(\rho^{\mathrm{s}} \epsilon\right)^{\frac{1}{2}}\left\|\dot{\boldsymbol{d}}\left(t_{n}\right)-\dot{\boldsymbol{d}}_{h}^{n}\right\|_{0, \Sigma}+\left\|\boldsymbol{d}\left(t_{n}\right)-\boldsymbol{d}_{h}^{n}\right\|_{\mathrm{e}}+\left(\sum_{m=r^{\star}+1}^{n} \tau\left\|\dot{\boldsymbol{d}}\left(t_{m}\right)-\dot{\boldsymbol{d}}_{h}^{m}\right\|_{\mathrm{v}}^{2}\right)^{\frac{1}{2}}
\end{aligned}
$$


for $n \geq r^{\star}+1$. The following result states an priori error estimate for the fully discrete explicit Robin-Neumann schemes introduced in Section 4.2.

Theorem 3 Let $(\boldsymbol{u}, p, \boldsymbol{d}, \dot{\boldsymbol{d}})$ be the solution of the coupled problem (19) and $\left\{\left(\boldsymbol{u}_{h}^{n}, p_{h}^{n}, \boldsymbol{d}_{h}^{n}, \dot{\boldsymbol{d}}_{h}^{n}\right)\right\}_{n \geq r^{\star}+1}$ be the discrete solution given by Algorithm 3 with initial data $\left(\boldsymbol{u}_{h}^{0}, \boldsymbol{d}_{h}^{0}, \dot{d}_{h}^{0}\right)=\left(\boldsymbol{P}_{h} \boldsymbol{u}^{0}, \boldsymbol{\pi}_{h}^{\mathrm{e}} \boldsymbol{d}^{0}, \boldsymbol{\pi}_{h}^{\mathrm{v}} \dot{\boldsymbol{d}}^{0}\right)$. The initialization procedure of Remark 4 is considered for the schemes with with extrapolation $\left(r^{\star} \geq 1\right)$. Suppose that (32)-(34) hold and that the exact solution has the regularity (35)-(36). For the scheme with $r^{\star}=2$ we assume, in addition, that (3) and the stability condition (29) hold. Then, we have the following error estimates, for $n \geq r^{\star}+1$ such as $n \tau<T$ :

$$
\mathcal{E}_{h}^{n} \lesssim c_{1}^{\star} h^{k}+c_{2}^{\star} h^{\tilde{l}}+c_{3}^{\star} \tau+c_{4}^{\star} \begin{cases}\tau^{\frac{1}{2}} & \text { if } r^{\star}=0, \\ \tau & \text { if } r^{\star}=1, \\ \tau^{2} & \text { if } r^{\star}=2 .\end{cases}
$$

Here, the symbols $\left\{c_{i}^{\star}\right\}_{i=1}^{4}$ denote positive constants independent of $h$ and $\tau$, but which depend on the physical parameters and on the regularity of $(\boldsymbol{u}, p, \boldsymbol{d}, \dot{\boldsymbol{d}})$.

Proof 3 See C.

Theorem 3 shows that, for regular enough solutions, the proposed explicit Robin-Neumann schemes converge to the solution of (1)-(2). The last term of (37) represents the truncation error introduced by the Robin-Neumann splitting, that is, the time-consistency of the kinematic perturbation in (25). For the variant without extrapolation, the error estimate (37) predicts a sub-optimal $\mathcal{O}\left(\tau^{\frac{1}{2}}\right)$ time-convergence rate in the energy-norm. This is due to the low-order consistency of the perturbation in the case $r^{\star}=0$. On the contrary, for the variants with extrapolation the consistency of the perturbations scale as $\mathcal{O}(\tau)$ and $\mathcal{O}\left(\tau^{2}\right)$, respectively. An overall optimal convergence-rate $\mathcal{O}\left(h^{k}+h^{\tilde{l}}+\tau\right)$ is hence recovered.

In view of Theorems 1 and 3 , the explicit Robin-Neumann scheme with $r^{\star}=1$ simultaneously yields stability and optimal accuracy with no restrictions on the discretization parameters nor on the polynomial order.

Remark 11 Theorem 3 generalizes the error estimates reported in [21, Section 5] to the case $\boldsymbol{L}^{\mathrm{v}} \neq 0 . \diamond$

\section{Formulation in the non-linear case}

In this section we extend the explicit Robin-Neumann paradigm of Section 3.1 to the case of a nonlinear fluid-structure problem, involving a viscous incompressible fluid and thin-walled structure. The fluid is described by the incompressible Navier-Stokes equations in ALE formalism (see, e.g., [27, Chapter 3]) and the structure by a non-linear Reissner-Mindlin shell model (see, e.g., [13, 4]).

\subsection{The non-linear coupled problem}

Let $\Omega \cup \Sigma$ be a reference configuration of the system. The current configuration of the fluid domain, $\Omega(t)$, is parametrized by the ALE map $\mathcal{A} \stackrel{\text { def }}{=} \boldsymbol{I}_{\Omega}+\boldsymbol{d}^{\mathrm{f}}$ as $\Omega(t)=\mathcal{A}(\Omega, t)$, where $\boldsymbol{d}^{\mathrm{f}}$ : $\Omega \times \mathbb{R}^{+} \rightarrow \mathbb{R}^{d}$ stands for the displacement of the fluid domain. In practice, $\boldsymbol{d}^{\mathrm{f}}=\operatorname{Ext}\left(\left.\boldsymbol{d}\right|_{\Sigma}\right)$, where $\operatorname{Ext}(\cdot)$ denotes any reasonable lifting operator from the (reference) interface $\Sigma$ into the (reference) fluid domain $\Omega$. For instance, an harmonic lifting operator is used in the numerical experiments reported in Section 6. 
The non-linear fluid-structure problem under consideration reads as follows: Find the fluid domain displacement $\boldsymbol{d}^{\mathrm{f}}: \Omega \times \mathbb{R}^{+} \rightarrow \mathbb{R}^{d}$, the fluid velocity $\boldsymbol{u}: \Omega \times \mathbb{R}^{+} \rightarrow \mathbb{R}^{d}$, the fluid pressure $p: \Omega \times \mathbb{R}^{+} \rightarrow \mathbb{R}^{d}$ the solid displacement $\boldsymbol{d}: \Sigma \times \mathbb{R}^{+} \rightarrow \mathbb{R}^{d}$ and the surface vector field $\boldsymbol{\theta}:$ $\Sigma \times \mathbb{R}^{+} \rightarrow \mathbb{R}^{3}$ such that

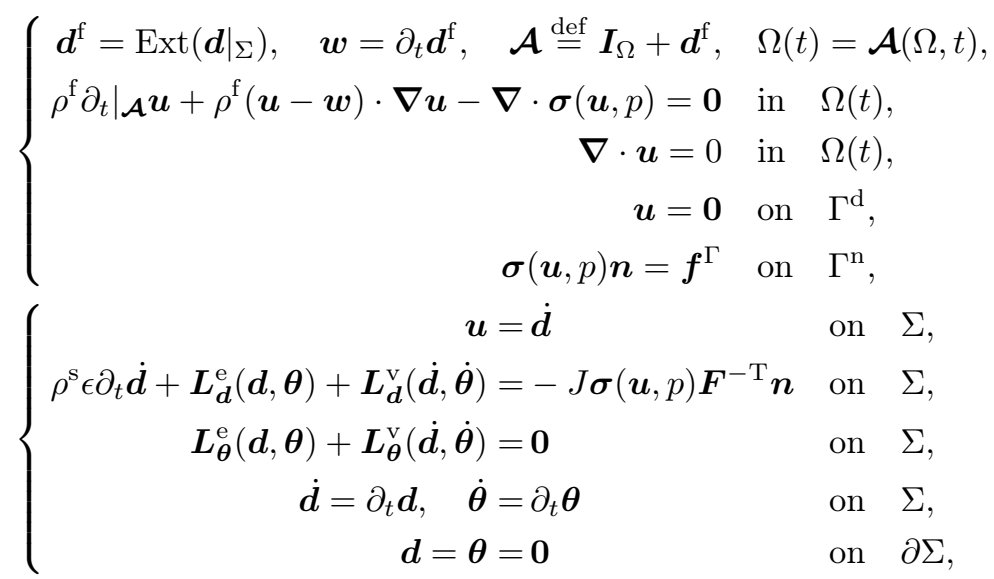

where $\left.\partial_{t}\right|_{\mathcal{A}}$ represents the ALE time derivative, $\boldsymbol{F} \stackrel{\text { def }}{=} \boldsymbol{\nabla} \mathcal{A}$ the fluid domain gradient of deformation and $J \stackrel{\text { def }}{=} \operatorname{det} \boldsymbol{F}$ the Jacobian. The surface elastic and viscous operators, $\left(\boldsymbol{L}_{\boldsymbol{d}}^{\mathrm{e}}, \boldsymbol{L}_{\boldsymbol{\theta}}^{\mathrm{e}}\right)$ and $\left(\boldsymbol{L}_{\boldsymbol{d}}^{\mathrm{v}}, \boldsymbol{L}_{\boldsymbol{\theta}}^{\mathrm{v}}\right)$, can be non-linear. As usual, a field defined in the reference fluid domain, $\Omega$, is evaluated in the current fluid domain, $\Omega(t)$, by composition with $\mathcal{A}^{-1}(\cdot, t)$.

\subsection{Explicit Robin-Neumann schemes}

The proposed fully explicit coupling schemes combine an explicit treatment of the interface geometrical compatibility (38) ${ }_{1}$ with an explicit Robin-Neumann treatment of the interface kinematical and kinetic coupling (39) 1,2 . Following the initial argument of Section 3, the interface Robin consistency of the coupled problem (38)-(39) is given by the relation

$$
J \boldsymbol{\sigma}(\boldsymbol{u}, p) \boldsymbol{F}^{-\mathrm{T}} \boldsymbol{n}+\rho^{\mathrm{s}} \epsilon \partial_{t} \boldsymbol{u}=-\boldsymbol{L}_{\boldsymbol{d}}^{\mathrm{e}}(\boldsymbol{d}, \boldsymbol{\theta})-\boldsymbol{L}_{\boldsymbol{d}}^{\mathrm{v}}(\dot{\boldsymbol{d}}, \dot{\boldsymbol{\theta}}) \quad \text { on } \quad \Sigma .
$$

Nevertheless, in order to avoid the extrapolation of the viscoelastic non-linear terms in the right-hand side, we instead consider the non-linear counterpart of the alternative condition given by (12), that is,

$$
J \boldsymbol{\sigma}(\boldsymbol{u}, p) \boldsymbol{F}^{-\mathrm{T}} \boldsymbol{n}+\frac{\rho^{\mathrm{s}} \epsilon}{\tau} \boldsymbol{u}=\frac{\rho^{\mathrm{s}} \epsilon}{\tau} \dot{\boldsymbol{d}}+J \boldsymbol{\sigma}(\boldsymbol{u}, p) \boldsymbol{F}^{-\mathrm{T}} \boldsymbol{n} \quad \text { on } \quad \Sigma
$$

which motivates the following intrinsic explicit Robin condition for the fluid

$$
J^{n} \boldsymbol{\sigma}\left(\boldsymbol{u}^{n}, p^{n}\right)\left(\boldsymbol{F}^{n}\right)^{-\mathrm{T}} \boldsymbol{n}+\frac{\rho^{\mathrm{s}} \epsilon}{\tau} \boldsymbol{u}^{n}=\frac{\rho^{\mathrm{s}} \epsilon}{\tau}\left(\dot{\boldsymbol{d}}^{n-1}+\tau \partial_{\tau} \dot{\boldsymbol{d}}^{\star}\right)+\boldsymbol{t}^{\star} \quad \text { on } \quad \Sigma,
$$

where $\dot{\boldsymbol{d}}^{\star}=\mathbf{0}, \dot{\boldsymbol{d}}^{n-1}, 2 \dot{\boldsymbol{d}}^{n-1}+\dot{\boldsymbol{d}}^{n-2}$ and

$$
\boldsymbol{t}^{\star}=\left\{\begin{array}{l}
\mathbf{0} \\
J^{n-1} \boldsymbol{\sigma}\left(\boldsymbol{u}^{n-1}, p^{n-1}\right)\left(\boldsymbol{F}^{n-1}\right)^{-\mathrm{T}} \boldsymbol{n}, \\
2 J^{n-1} \boldsymbol{\sigma}\left(\boldsymbol{u}^{n-1}, p^{n-1}\right)\left(\boldsymbol{F}^{n-1}\right)^{-\mathrm{T}} \boldsymbol{n}-J^{n-2} \boldsymbol{\sigma}\left(\boldsymbol{u}^{n-2}, p^{n-2}\right)\left(\boldsymbol{F}^{n-2}\right)^{-\mathrm{T}} \boldsymbol{n},
\end{array}\right.
$$

$\mathrm{RR} \mathrm{n}^{\circ} 8224$ 


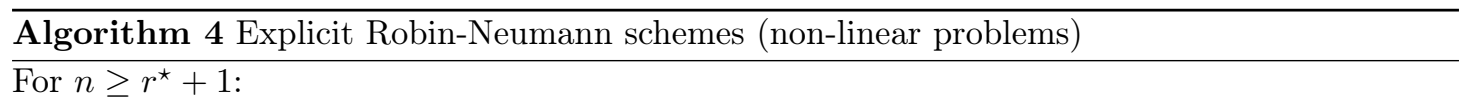

1. Fluid domain update:

$$
\boldsymbol{d}^{\mathrm{f}, n}=\operatorname{Ext}\left(\left.\boldsymbol{d}^{n-1}\right|_{\Sigma}\right), \quad \boldsymbol{w}^{n}=\partial_{\tau} \boldsymbol{d}^{\mathrm{f}, n}, \quad \mathcal{A}^{n} \stackrel{\text { def }}{=} \boldsymbol{I}_{\Omega}+\boldsymbol{d}^{\mathrm{f}, n}, \quad \Omega^{n} \stackrel{\text { def }}{=} \mathcal{A}^{n}(\Omega)
$$

and we set $\boldsymbol{F}^{n}=\nabla \mathcal{A}^{n}$ and $J^{n}=\operatorname{det} \boldsymbol{F}^{n}$.

2. Fluid step: find $\boldsymbol{u}^{n}: \Omega \times \mathbb{R}^{+} \rightarrow \mathbb{R}^{3}$ and $p^{n}: \Omega \times \mathbb{R}^{+} \rightarrow \mathbb{R}$ such that

$$
\left\{\begin{array}{rccc}
\rho^{\mathrm{f}} \partial_{\tau} \mid \mathcal{A} \boldsymbol{u}^{n}+\rho^{\mathrm{f}}\left(\boldsymbol{u}^{n-1}-\boldsymbol{w}^{n}\right) \cdot \boldsymbol{\nabla} \boldsymbol{u}^{n}-\boldsymbol{\nabla} \cdot \boldsymbol{\sigma}\left(\boldsymbol{u}^{n}, p^{n}\right)=\mathbf{0}, & \text { in } & \Omega^{n}, \\
\operatorname{div} \boldsymbol{u}^{n}=0 & \text { in } & \Omega^{n}, \\
\boldsymbol{u}^{n}=\mathbf{0} & \text { on } & \Gamma^{\mathrm{d}}, \\
\boldsymbol{\sigma}\left(\boldsymbol{u}^{n}, p^{n}\right) \boldsymbol{n}=\boldsymbol{f}^{\Gamma} & \text { on } & \Gamma^{\mathrm{n}}, \\
J^{n} \boldsymbol{\sigma}\left(\boldsymbol{u}^{n}, p^{n}\right)\left(\boldsymbol{F}^{n}\right)^{-\mathrm{T}} \boldsymbol{n}+\frac{\rho^{\mathrm{s}} \epsilon}{\tau} \boldsymbol{u}^{n}=\frac{\rho^{\mathrm{s}} \epsilon}{\tau}\left(\dot{\boldsymbol{d}}^{n-1}+\tau \partial_{\tau} \dot{\boldsymbol{d}}^{\star}\right)+\boldsymbol{t}^{*} & \text { on } & \Sigma,
\end{array}\right.
$$

with the fluid stress extrapolations $\boldsymbol{t}^{\star}$ given by (42).

3. Solid step: find $\boldsymbol{d}^{n}: \Sigma \times \mathbb{R}^{+} \rightarrow \mathbb{R}^{3}$ and $\boldsymbol{\theta}^{n}: \Sigma \times \mathbb{R}^{+} \rightarrow \mathbb{R}^{3}$ such that

$$
\left\{\begin{array}{rlrl}
\rho^{\mathrm{s}} \epsilon \partial_{\tau} \dot{\boldsymbol{d}}^{n}+\boldsymbol{L}_{\boldsymbol{d}}^{\mathrm{e}}\left(\boldsymbol{d}^{n}, \boldsymbol{\theta}^{n}\right)+\boldsymbol{L}_{\boldsymbol{d}}^{\mathrm{v}}\left(\dot{\boldsymbol{d}}^{n}, \dot{\boldsymbol{\theta}}^{n}\right) & =-J^{n} \boldsymbol{\sigma}\left(\boldsymbol{u}^{n}, p^{n}\right)\left(\boldsymbol{F}^{n}\right)^{-\mathrm{T}} \boldsymbol{n} & & \text { on } \quad \Sigma, \\
\boldsymbol{L}_{\boldsymbol{\theta}}^{\mathrm{e}}\left(\boldsymbol{d}^{n}, \boldsymbol{\theta}^{n}\right)+\boldsymbol{L}_{\boldsymbol{\theta}}^{\mathrm{v}}\left(\dot{\boldsymbol{d}}^{n}, \dot{\boldsymbol{\theta}}^{n}\right) & =\mathbf{0} & \text { on } \quad \Sigma, \\
\dot{\boldsymbol{d}}=\partial_{\tau} \boldsymbol{d}^{n}, \quad \dot{\boldsymbol{\theta}}^{n} & =\partial_{\tau} \boldsymbol{\theta}^{n} & \text { on } \Sigma, \\
\boldsymbol{d}^{n}=\boldsymbol{\theta}^{n} & =\mathbf{0} & \text { on } \quad \partial \Sigma .
\end{array}\right.
$$

stands, respectively, for the zeroth- $\left(r^{\star}=0\right)$, first- $\left(r^{\star}=1\right)$ and second-order $\left(r^{\star}=2\right)$ extrapolations of the solid velocity, $\dot{\boldsymbol{d}}$, and of the interface fluid stress in the reference configuration, $J \boldsymbol{\sigma}(\boldsymbol{u}, p) \boldsymbol{F}^{-\mathrm{T}} \boldsymbol{n}$. The explicit Robin condition (41) corresponds to the non-linear counterpart of (11). The resulting explicit coupling schemes are detailed in Algorithm 4.

It should be noted that the geometrical quantities involved in (41) and (42) do not need any specific treatment and appear because the interface relation (40) is formulated in the reference configuration. In practice, the interface fluid stresses are consistently evaluated as variational residuals in the deformed configurations, while the solid inertial contributions are integrated in the reference configuration. These hybrid characteristics of the explicit Robin condition (41) are handled in a natural manner by the ALE formalism.

\section{$6 \quad$ Numerical experiments}

In order to assess the behavior and robustness of the explicit Robin-Neumann schemes, we have considered a number of fluid-structure interaction examples from the literature. Section 6.1 presents a convergence study in 2D, using the linear model problem (1)-(2). Numerical results with 3D geometries and non-linear models (38)-(39) are reported in the subsequent Sections 6.26.5 . 


\subsection{Convergence study in a two-dimensional test-case}

The first example simulates a pressure wave propagation within a compliant channel in 2D (see, e.g., $[32,21])$. We couple the Stokes equations with a damped generalized string model. More precisely, in (2) we take

$$
\boldsymbol{d}=\left(\begin{array}{c}
0 \\
\boldsymbol{d}_{y}
\end{array}\right), \quad \boldsymbol{L}^{\mathrm{e}} \boldsymbol{d}=\left(\begin{array}{c}
0 \\
-\lambda_{1} \partial_{x x} \boldsymbol{d}_{y}+\lambda_{0} \boldsymbol{d}_{y}
\end{array}\right), \quad \boldsymbol{L}^{\mathrm{v}} \dot{\boldsymbol{d}}=\left(\begin{array}{c}
0 \\
\alpha \rho^{\mathrm{s}} \epsilon \dot{\boldsymbol{d}}_{y}-\beta \lambda_{1} \partial_{x x} \dot{\boldsymbol{d}}_{y}
\end{array}\right),
$$

with $\lambda_{1} \stackrel{\text { def }}{=} E \epsilon /(2(1+\nu))$ and $\lambda_{0} \stackrel{\text { def }}{=} E \epsilon /\left(R^{2}\left(1-\nu^{2}\right)\right)$. As usual, here $E$ denotes the Young modulus and $\nu$ the Poisson ratio of the solid. All the quantities will be given in the CGS system. The fluid domain and the fluid-solid interface are, respectively, $\Omega=[0, L] \times[0, R], \quad \Sigma=[0, L] \times\{R\}$, with $L=6$ and $R=0.5$. At $x=0$ we impose a sinusoidal normal traction of maximal amplitude $2 \cdot 10^{4}$

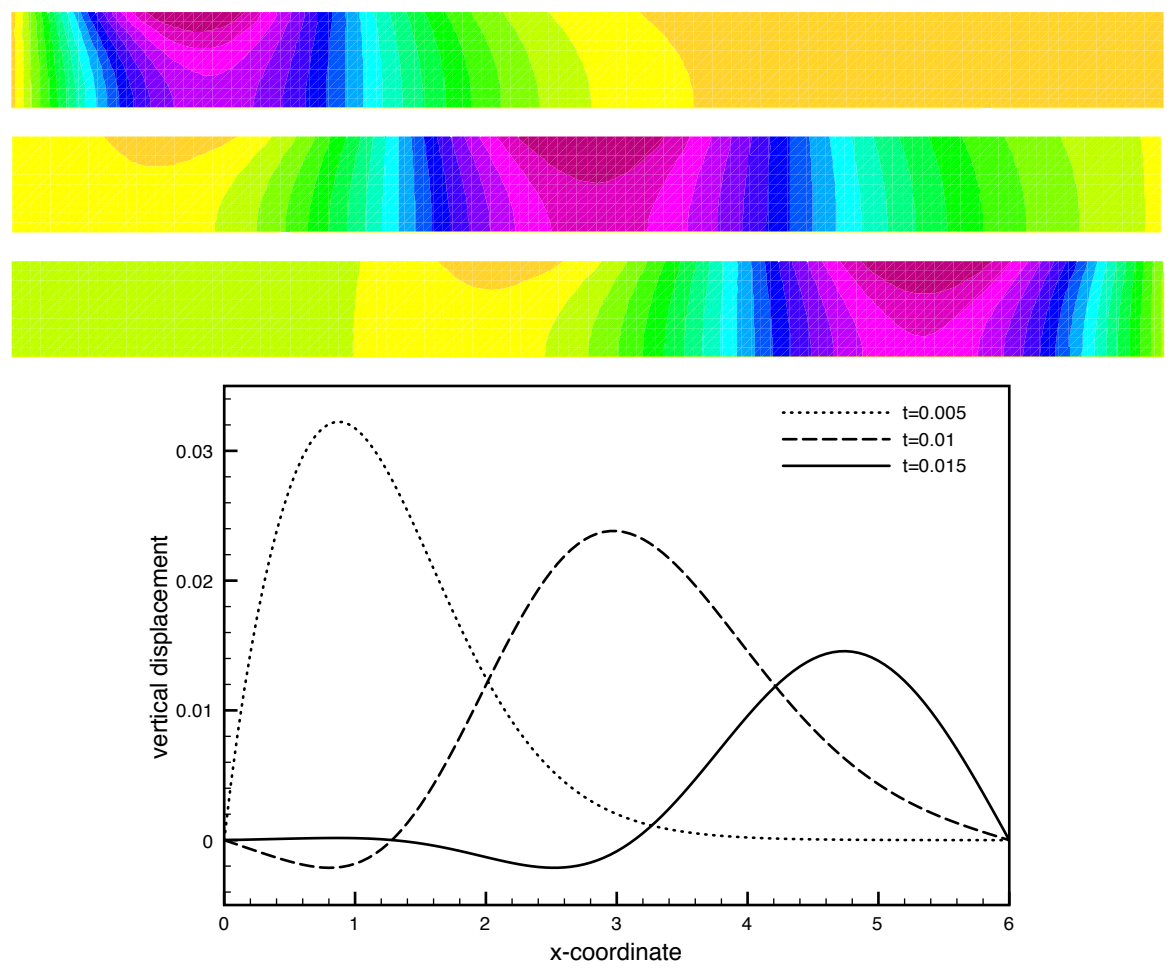

Figure 1: Snapshots of the fluid pressure and solid displacement at $t=0.005,0.01,0.015$ (from top to bottom). Algorithm 3 with first-order extrapolation, $\tau=10^{-4}$ and $h=0.05$.

during $5 \cdot 10^{-3}$ seconds, corresponding to half a period. Zero traction is enforced at $x=6$ and a symmetry condition is applied on the lower wall $y=0$. The solid is clamped at its extremities, $x=0, L$. The fluid physical parameters are given by $\rho^{\mathrm{f}}=1.0$ and $\mu=0.035$, while for the solid we have $\rho^{\mathrm{s}}=1.1, \epsilon=0.1, E=0.75 \times 10^{6}, \nu=0.5, \alpha=1$ and $\beta=10^{-3}$.

Continuous piece-wise affine approximations are considered for both the fluid and the structure, with the symmetric pressure stabilization method introduced in [6]. We have reported in Figure 1 a few snapshots of the pressure and displacement fields obtained using Algorithm 3 with the first-order extrapolation, $\tau=10^{-4}$ and $h=0.05$. All the computations have been performed 


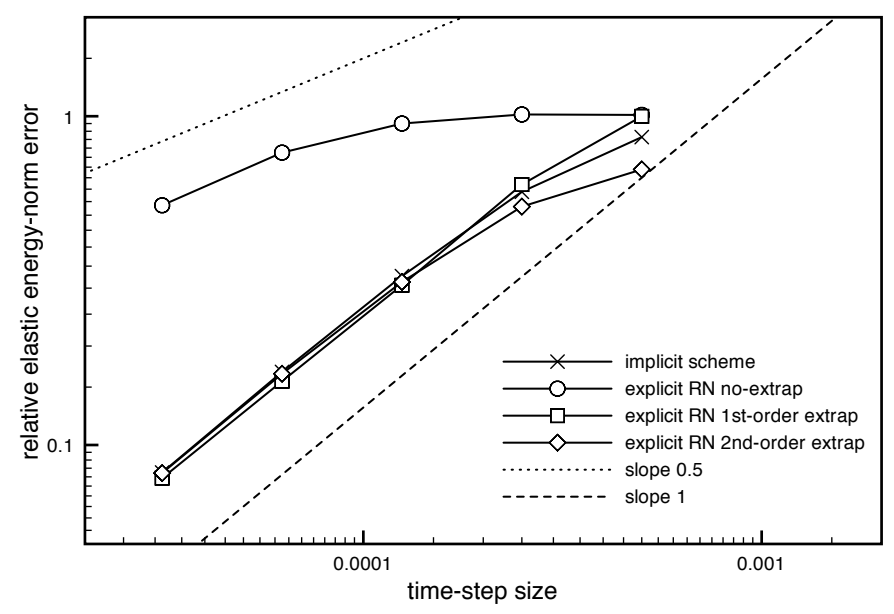

Figure 2: Time-convergence history of the displacement at $t=0.015$, with $h=\mathcal{O}(\tau)$.

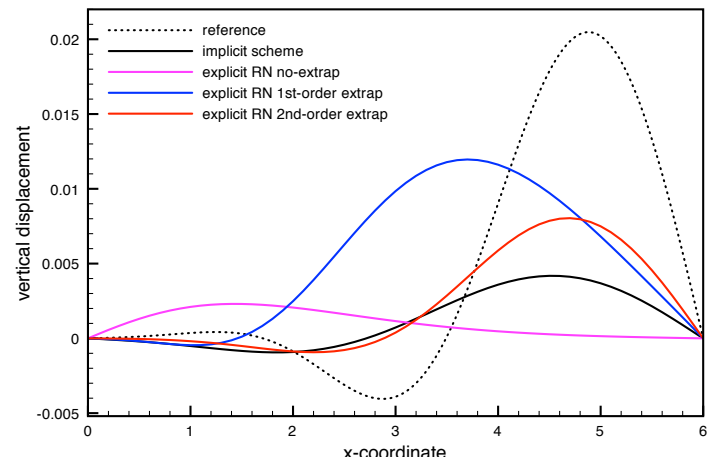

(a) $i=0$.

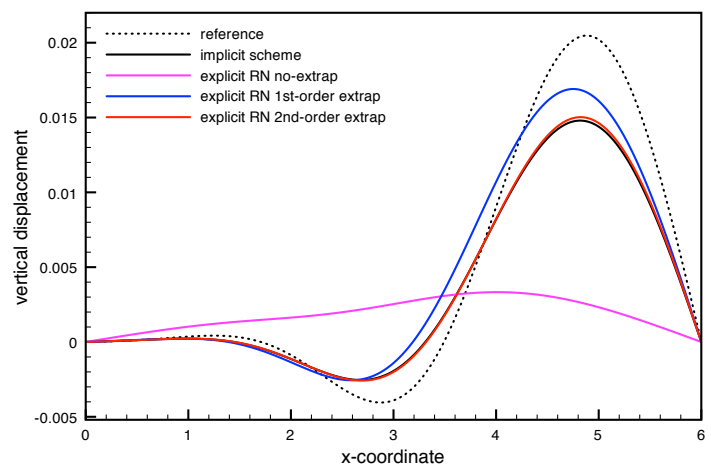

(c) $i=2$.

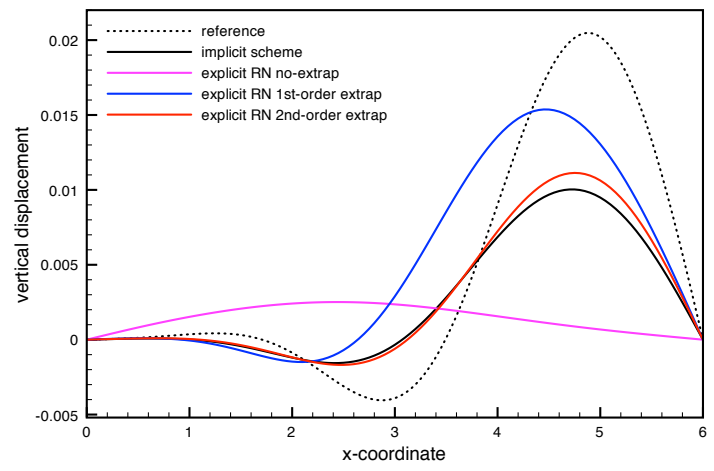

(b) $i=1$.

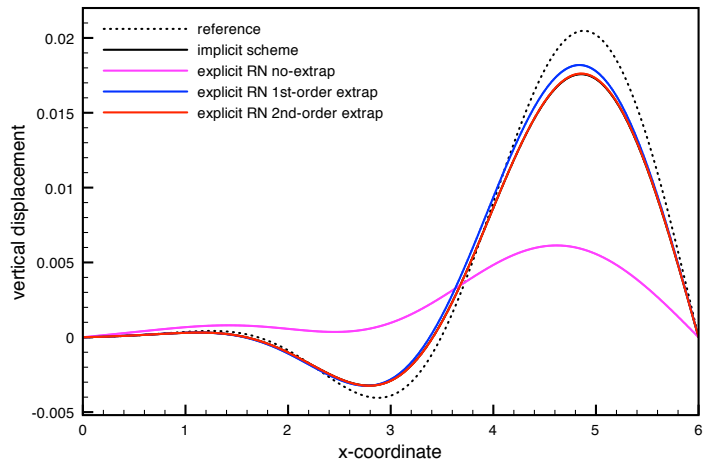

(d) $i=3$.

Figure 3: Comparison of the solid displacements at $t=0.015$ for different levels of $(\tau, h)$ refinement, given by (43) with $i=0, \ldots, 3$.

with FreeFem $++[51]$. The numerical solution remains stable, as predicted by Theorem 1, and 
a propagating pressure-wave is observed.

In order to highlight the $h$-uniformity of the convergence in time, we have refined both in time and in space at the same rate, with the following set of discrete parameters:

$$
(\tau, h) \in\left\{2^{-i}\left(5 \cdot 10^{-4}, 0.1\right)\right\}_{i=0}^{4} .
$$

A reference solution has been generated using a fully implicit scheme with a high space-time grid resolution $\left(h=3.125 \times 10^{-3}, \tau=10^{-6}\right)$. Figure 2 reports the corresponding time-convergence history of the solid displacement at time $t=0.015$, in the relative elastic energy-norm, for the proposed explicit Robin-Neumann schemes (with and without extrapolation) and the implicit scheme. The explicit Robin-Neumann schemes with first- and second-order extrapolation yield an overall $\mathcal{O}(\tau)$ optimal accuracy, while a sub-optimal $\mathcal{O}\left(\tau^{\frac{1}{2}}\right)$ rate is observed without extrapolation. Thus, in agreement with the error estimates provided by Theorem 3. The impact of the suboptimal rate is clearly visible in Figure 3, where we have displayed the interface displacements associated to Figure 2 (first four points of each curve). For comparison purposes, the reference displacement is also shown. Observe that, even with the smallest time-steps sizes (e.g., Figure $3(\mathrm{~d})$ ), the Robin-Neumann scheme without extrapolation provides a rather poor approximation. On the contrary, the extrapolated variants are able to retrieve the accuracy of the implicit coupling scheme. In the case of the coupling with an undamped structure (i.e., with $\alpha=\beta=0$ ), a similar behavior was observed in [21].

\subsection{Pressure wave propagation in a straight tube}

The second example is the three-dimensional test-case proposed in [26] (see also [27, Chapter 12]). The fluid domain is a straight tube of radius $R=0.5$ and of length $L=5$. All the units are given in the CGS system. The fluid is governed by the incompressible Navier-Stokes equations in ALE formism, discretized in space with $\mathbb{Q}_{1} / \mathbb{Q}_{1}$ finite elements and a SUPG/PSPG stabilized formulation. The vessel wall is described by the linear shell model (4) (we refer to [13, Section 4.2.1] for the details), discretized in space by quadrilateral MITC4 shell elements (see [13, Section 8.2.1]). The fluid physical parameters are given by $\rho^{\mathrm{f}}=1.0$ and $\mu=0.035$, while for the solid we have $\rho^{\mathrm{s}}=1.2, \epsilon=0.1, E=3 \times 10^{6}$ and $\nu=0.5$. We will consider the case of both a Rayleigh solid damping, viz., $\boldsymbol{L}_{\boldsymbol{d}}^{\mathrm{v}}(\dot{\boldsymbol{d}}, \dot{\boldsymbol{\theta}})=\alpha \rho^{\mathrm{s}} \epsilon \dot{\boldsymbol{d}}+\beta \boldsymbol{L}_{\boldsymbol{d}}^{\mathrm{e}}(\dot{\boldsymbol{d}}, \dot{\boldsymbol{\theta}}), \boldsymbol{L}_{\boldsymbol{\theta}}^{\mathrm{v}}(\dot{\boldsymbol{d}}, \dot{\boldsymbol{\theta}})=\beta \boldsymbol{L}_{\boldsymbol{\theta}}^{\mathrm{e}}(\dot{\boldsymbol{d}}, \dot{\boldsymbol{\theta}})$, with $\alpha=1, \beta=10^{-3}$, and an undamped solid $(\alpha=\beta=0)$.

The overall system is initially at rest. During the time-interval $\left[0,5 \cdot 10^{-3}\right]$, an over pressure of $1.3332 \times 10^{4}$ is imposed on the inlet boundary. The simulations are carried out over 150 time-steps of length $\tau=10^{-4}$. Figure 4 shows the fluid pressure and the solid deformation (amplified by a factor 10) at different time instants obtained with Algorithm 4 and the first-order extrapolation, $\tau=10^{-4}$. A stable pressure wave propagation is observed. The impact of the solid damping is also clearly visible.

For comparison purposes, Figure 5 reports the interface mid-point displacement magnitudes obtained with Algorithm 4 and the implicit coupling scheme. Once more, the Robin-Neumann scheme without extrapolation provides a very poor approximation, while the extrapolated variants are able to retrieve the accuracy of the implicit coupling scheme. It is worth noting that the solid damping has a rather limited impact on the numerical solution without extrapolation. This suggests that the artificial dissipation of the scheme (due to its $\mathcal{O}\left(\tau^{\frac{1}{2}}\right)$ loss in accuracy) is at least of the same order than the physical dissipation considered in the solid. Similar conclusions can be inferred from Figure 6, which reports the out-flows obtained with the different schemes. 

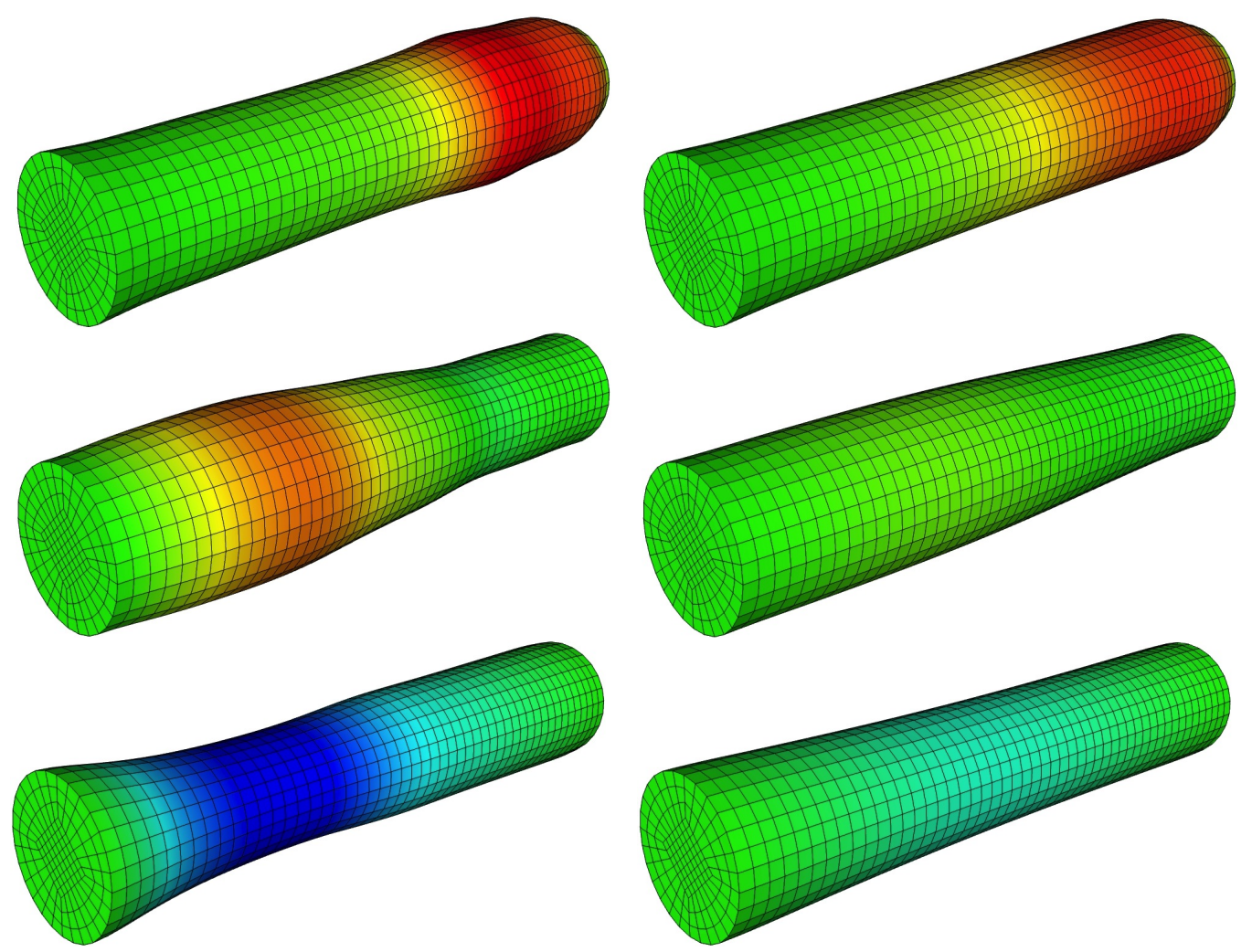

Figure 4: Snapshots of the fluid pressure at $t=0.005,0.01,0.015$ (from top to bottom). Algorithm 4 with first-order extrapolation, $\tau=10^{-4}$. Left: Shell model without damping $(\alpha=\beta=0)$. Right: Shell model with damping $\left(\alpha=1, \beta=10^{-3}\right)$.
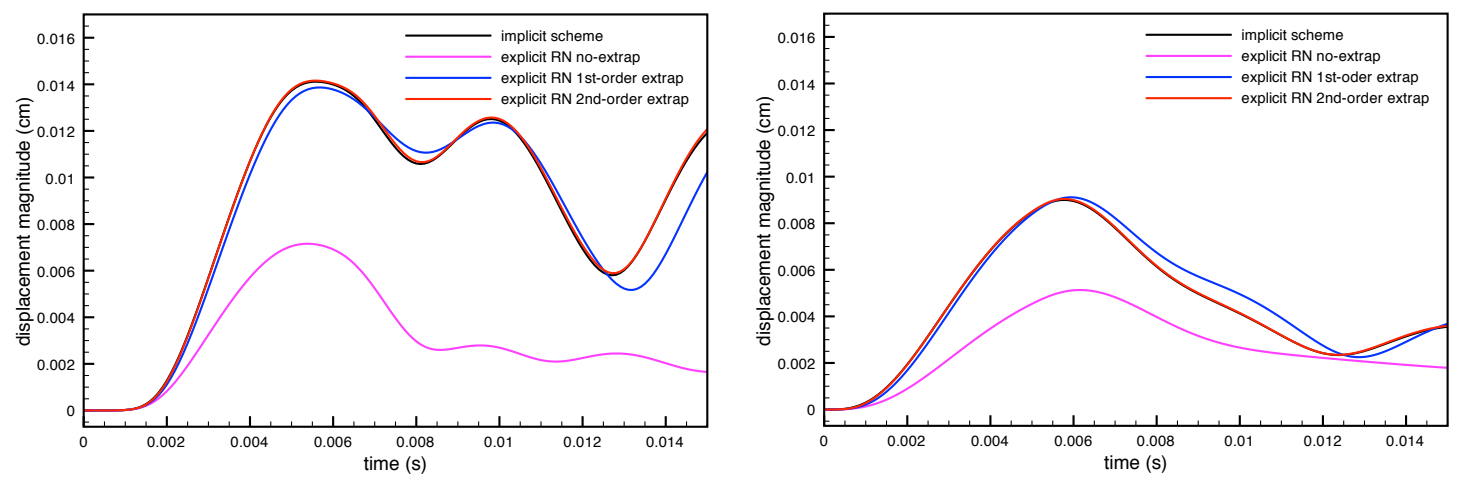

Figure 5: Comparison of the implicit and Robin-Neuman explicit coupling schemes: interface mid-point displacement magnitude vs. time. Left: Shell model without damping $(\alpha=\beta=0)$. Right: Shell model with damping $\left(\alpha=1, \beta=10^{-3}\right)$. 

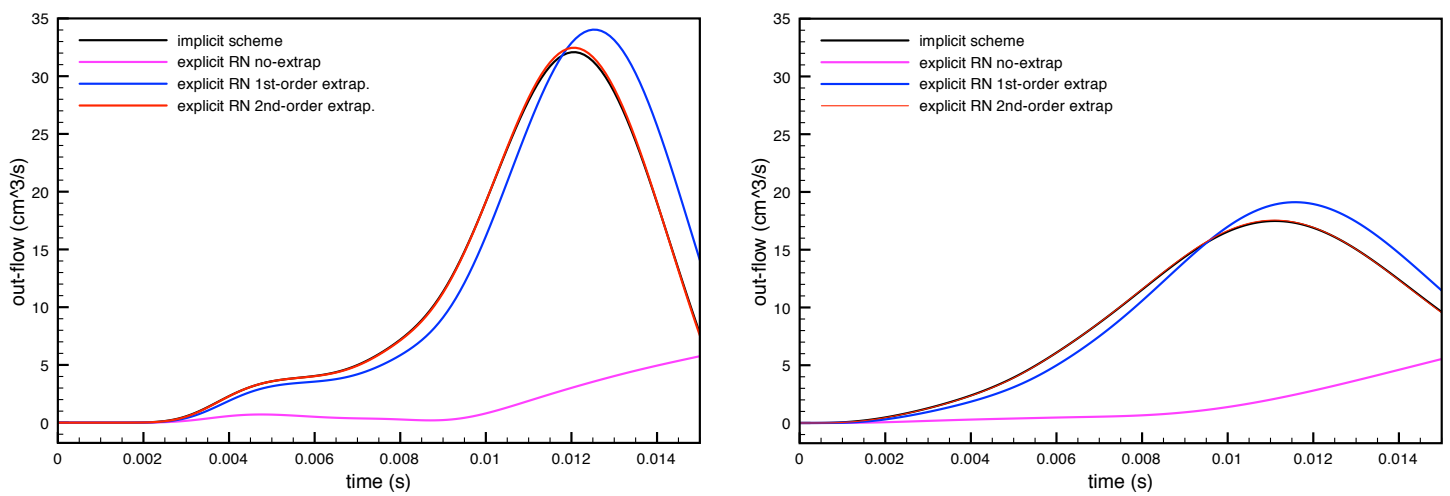

Figure 6: Comparison of the implicit and Robin-Neuman explicit coupling schemes: out-flow vs. time. Left: Shell model without damping $(\alpha=\beta=0)$. Right: Shell model with damping $(\alpha=1$, $\left.\beta=10^{-3}\right)$.

\subsection{Blood flow in an abdominal aortic aneurysm}

We consider the numerical example presented in [1] using an in vitro aneurysm geometry. The fluid computational domain is the idealized abdominal aortic aneurysm given in Figure 7 (left). We refer to [53] for the details. The whole compliant wall has a uniform thickness of 0.17 and length of 22.95. All the units are given in the CGS system. The fluid is governed by the
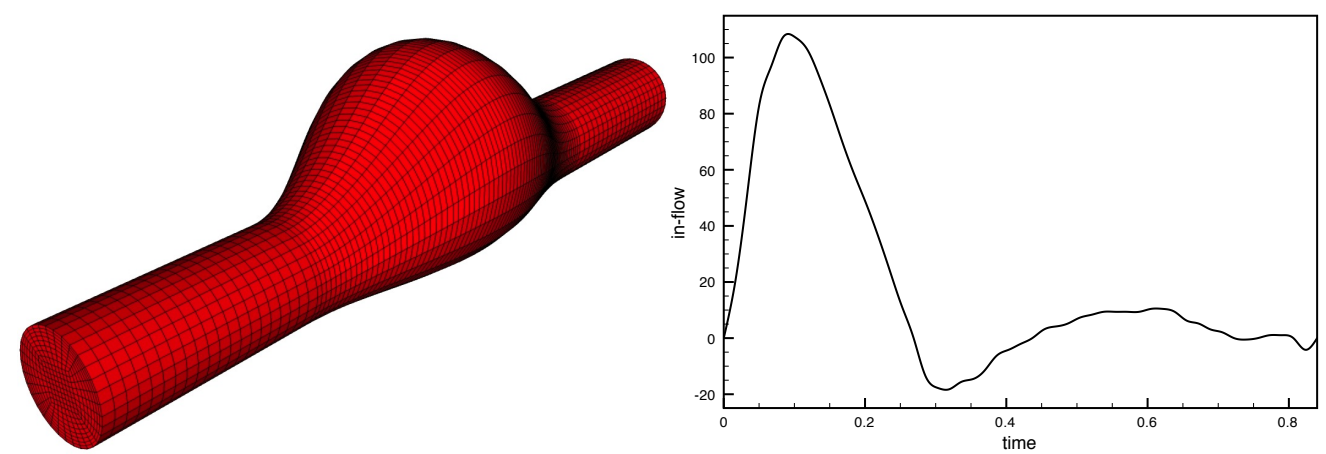

Figure 7: Aneurysm geometry (left) and in-flow rate data (right)

incompressible Navier-Stokes equations in ALE formism, discretized in space with $\mathbb{Q}_{1} / \mathbb{Q}_{1}$ finite elements and a SUPG/PSPG stabilized formulation. The vessel wall is described by the linear shell model (4), discretized in space by quadrilateral MITC4 shell elements. The fluid physical parameters are given by $\rho^{\mathrm{f}}=1.0$ and $\mu=0.035$, while for the solid we have $\rho^{\mathrm{s}}=1.2, \epsilon=0.1$, $E=3 \times 10^{6}, \nu=0.5, \alpha=1$ and $\beta=10^{-3}$. The overall system is initially at rest. An in-flow rate corresponding to a cardiac cycle, see Figure 7 (right), is imposed on the inlet boundary. A resistive-like boundary condition is prescribed on the outlet boundary, the value of the resistance being $R_{\text {out }}=6 \times 10^{2}$.

We have simulated 2000 time-steps of size $\tau=4.2 \times 10^{-4} \mathrm{~s}$, which corresponds to a full cardiac cycle. Figure 8 shows the fluid velocity and the solid deformation at different time instants obtained with Algorithm 4 and the first-order extrapolation. For comparison purposes, Figure 9 reports the interface mid-point displacement magnitudes and out-flow obtained with 

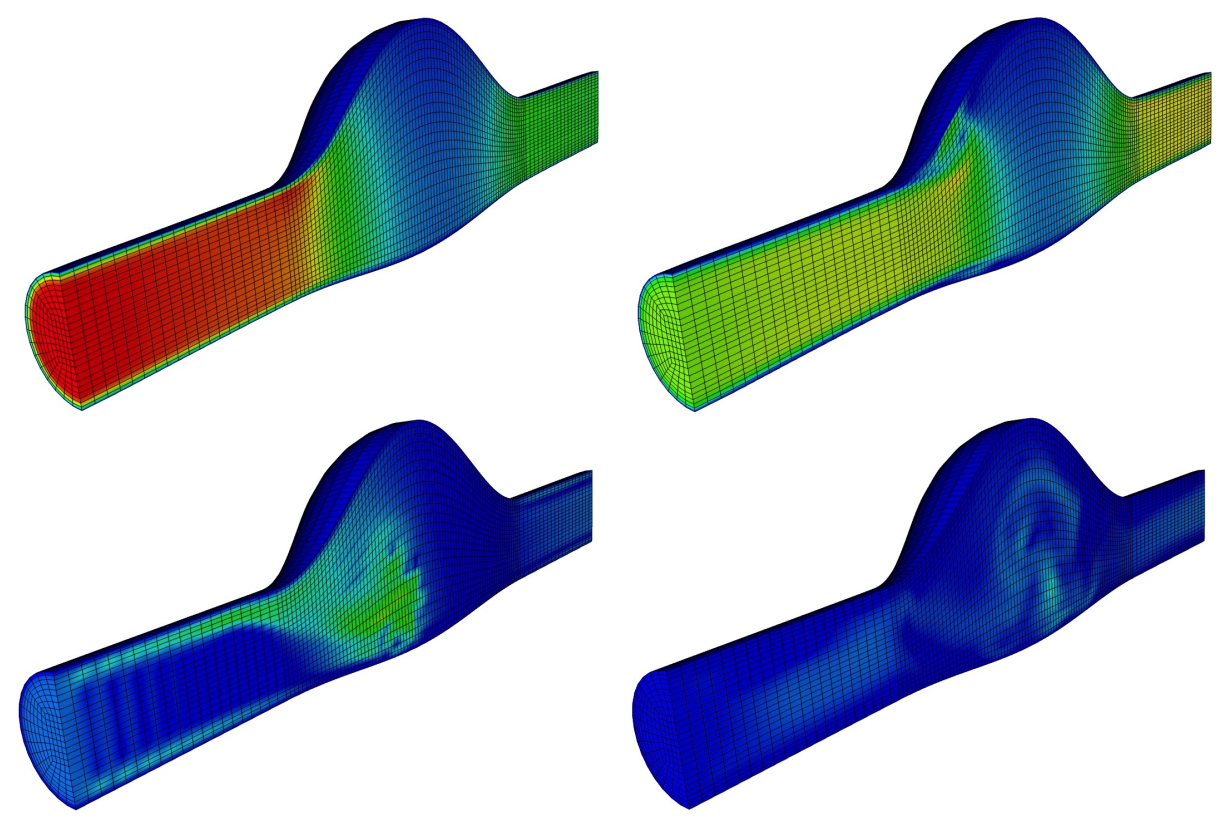

Figure 8: Snapshots of the fluid velocity $t=0.084,0.168,0.336,0.672$ (from left to right and top to bottom). Algorithm 4 with first-order extrapolation, $\tau=4.2 \cdot 10^{-4}$.
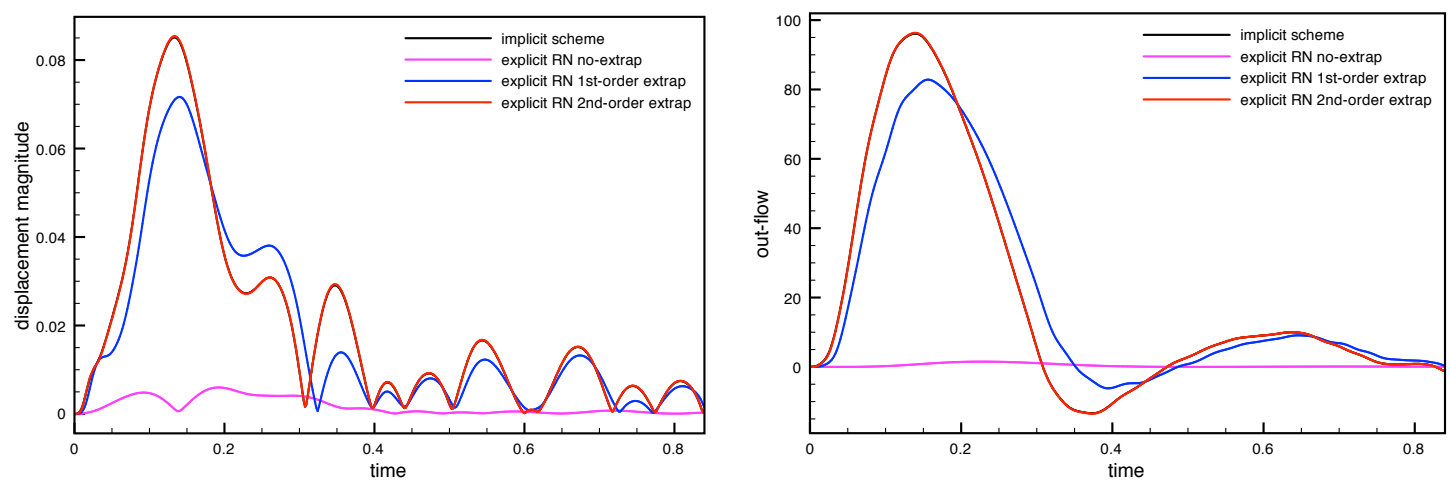

Figure 9: Comparison of the implicit and Robin-Neuman explicit coupling schemes: Left: Interface displacement. Right: Out-flow.

Algorithm 4 and the implicit coupling scheme. The Robin-Neumann schemes with first- and second-order extrapolation provide numerical solutions close to the implicit coupling scheme. The superior accuracy of the higher order kinematic perturbation introduced by the secondorder extrapolation is clearly visible. At last, the Robin-Neumann scheme without extrapolation yields an extremely poor approximation. Once more, this illustrates the striking impact of the $\mathcal{O}\left(\tau^{\frac{1}{2}}\right)$-loss on the accuracy of the scheme without extrapolation. 


\subsection{Driven cavity with flexible bottom}

We consider the classical shear-driven cavity problem with a flexible bottom (see, e.g., [28]). The fluid domain is the unit square $\Omega=[0,1] \times[0,1]$ with the fluid-solid interface on the lower boundary $\Sigma=[0,1] \times\{0\}$. All the units are given in the SI system. The overall system is initially at rest and an oscillating shear velocity profile is imposed on the upper boundary $\boldsymbol{u}(t)=$ $(1-\cos (0.4 \pi t), 0)^{\mathrm{T}}$ on $[0,1] \times\{1\}$. A non-slip condition is enforced on the portions $\{0,1\} \times[0,0.9]$
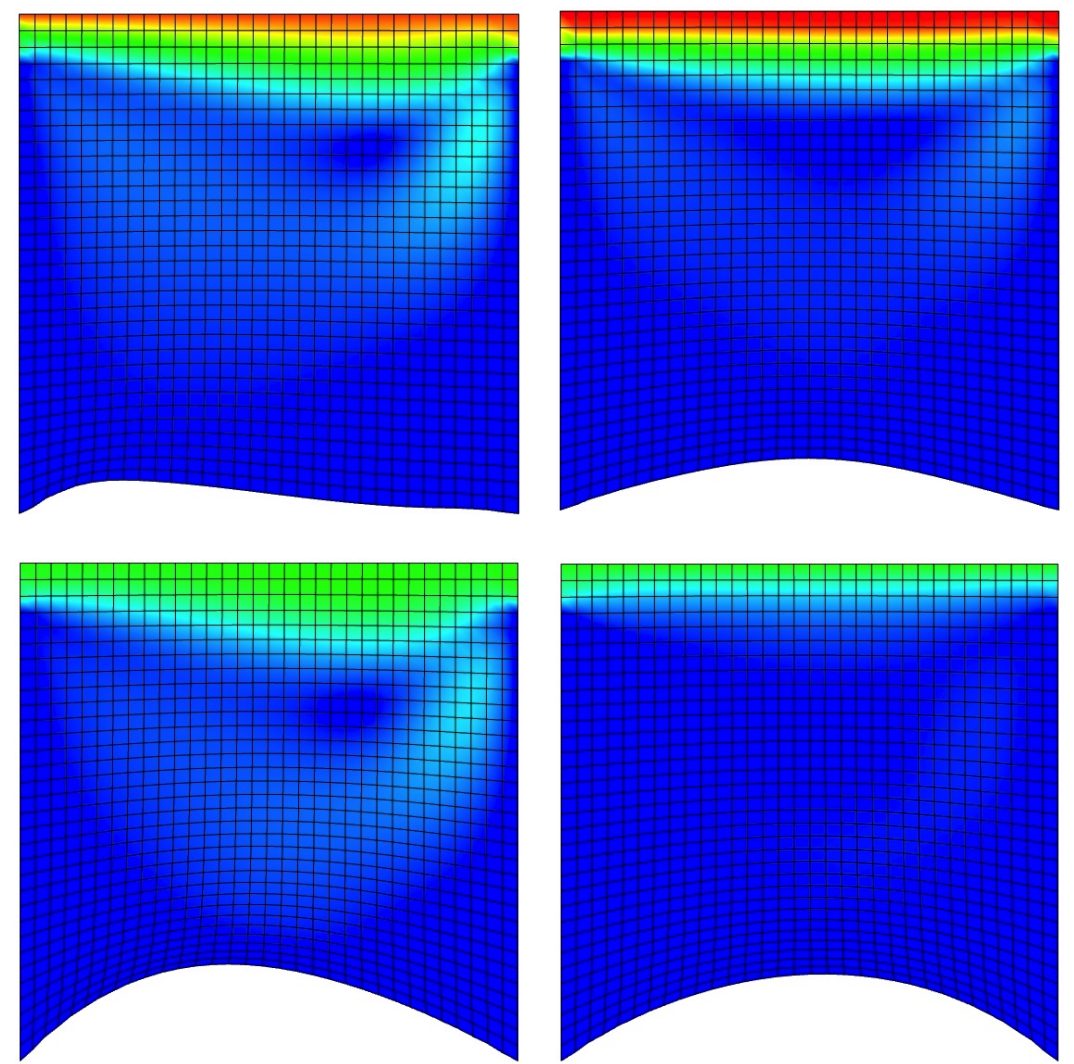

Figure 10: Snapshots of the fluid velocity magnitude at $t=3.5,8,14,21$ (from left to right and top to bottom). Algorithm 4 with first-order extrapolation, $\tau=0.1$.

of the lateral cavity walls. The system is described by the non-linear coupled problem (38)-(39), with a non-linear shell model and a Saint Venant-Kirchhoff constitutive law (see [13]). The fluid equations are discretized in space with $\mathbb{Q}_{1} / \mathbb{Q}_{1}$ finite elements and a SUPG/PSPG stabilized formulation. Quadrilateral MITC4 shell elements are considered for the structure. The fluid physical parameters are given by $\rho^{\mathrm{f}}=1.0$ and $\mu=0.01$, while for the solid (undamped) we have $\rho^{\mathrm{s}}=250, \epsilon=0.002, E=250$, and $\nu=0$.

The simulations are performed in three-dimensions, by imposing symmetry conditions along the extrusion direction, and carried out over 800 time-steps of size $\tau=0.1$. Figure 10 shows the fluid velocity and the solid deformation at different time instants obtained with Algorithm 4 and the first-order extrapolation.

For comparison purposes, Figure 11 reports the interface mid-point displacement magnitudes obtained with Algorithm 4 and the implicit coupling scheme. Once again, the superior accuracy 


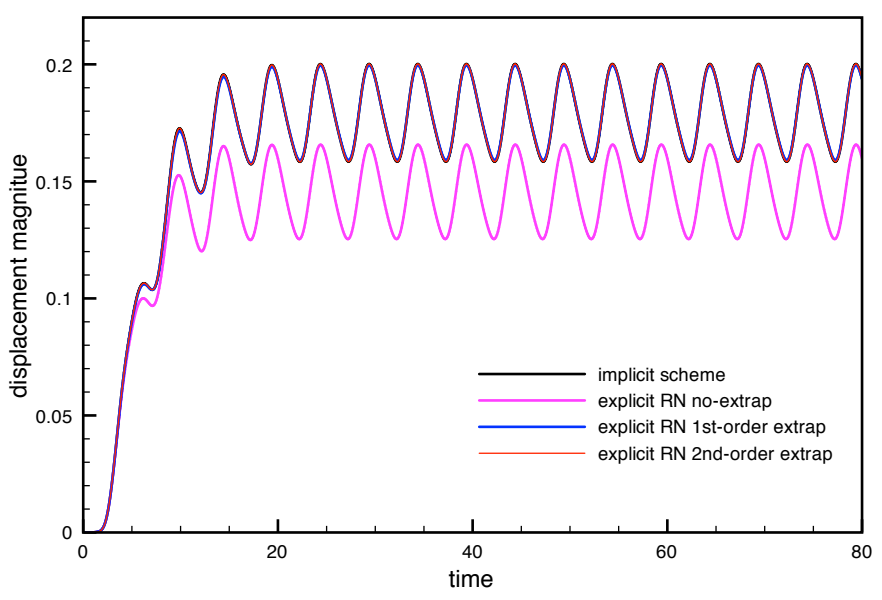

Figure 11: Comparison of the implicit and Robin-Neuman explicit coupling schemes: interface mid-point displacement magnitude $v s$. time.

of the Robin-Neumann schemes with first- and second-order extrapolation is noticeable.

\subsection{Damped structural instability}

The last example considers a bended fluid domain surrounded by two thin structures with different stiffness, as proposed in [40]. Both structures are fixed on their extremities. A constant parabolic velocity profile is prescribed on the left and right inflow boundaries, with maximal magnitudes 10 and 10.1, respectively (to avoid perfect symmetry). All the units are given in the SI system. Zero velocity is enforced on the remaining fluid boundaries. The fluid is loaded with the volume force $\boldsymbol{f}=(0,-1)^{\mathrm{T}}$. As in the previous example, the system is described by the non-linear coupled problem (38)-(39), with a non-linear shell model and a Saint Venant-Kirchhoff constitutive law. We consider also the same spatial discretization. The fluid physical parameters are given by $\rho^{\mathrm{f}}=1.0$ and $\mu=9$, while for the top and bottom (undamped) structures we have $\rho^{\mathrm{s}}=500, \epsilon=0.1, E_{\mathrm{top}}=9 \cdot 10^{5}, E_{\mathrm{bottom}}=9 \cdot 10^{8}$ and $\nu=0.3$. The simulations are performed in three-dimensions by imposing symmetry conditions along the extrusion direction.

A salient difficulty of this ballon-type problem is that it cannot be solved via standard Dirichlet-Neumann procedures, since the interface solid velocity does not necessarily satisfy the compatibility condition enforced by the incompressibility of the fluid (unless directly prescribed in the structure [40]). The explicit Robin-Neumann coupling schemes given by Algorithm 4 get rid of this issue without any extra adjustment. Indeed, the consistent perturbation of the kinematic coupling induced by the Robin-Neumann interface conditions removes the constraint on the interface solid velocity.

In Figure 12 we have reported the fluid velocity magnitude snapshots and the solid deformations at different time instants, obtained with Algorithm 4 (first-order extrapolation) and time-step length of $\tau=0.005$. As in [40], the deformation is first mainly visible in the upper (more flexible) structure and then, when the fluid pressure reaches a critical value, the lower structure buckles.

Figure 13 reports the interface mid-point displacement magnitude of the bottom structure obtained with Algorithm 4 and the implicit coupling scheme. The poor accuracy of the RobinNeumann scheme without extrapolation is striking: the lower order perturbation of the kinematic coupling yields an excess of mass-loss across the interface which prevents the buckling. On the 


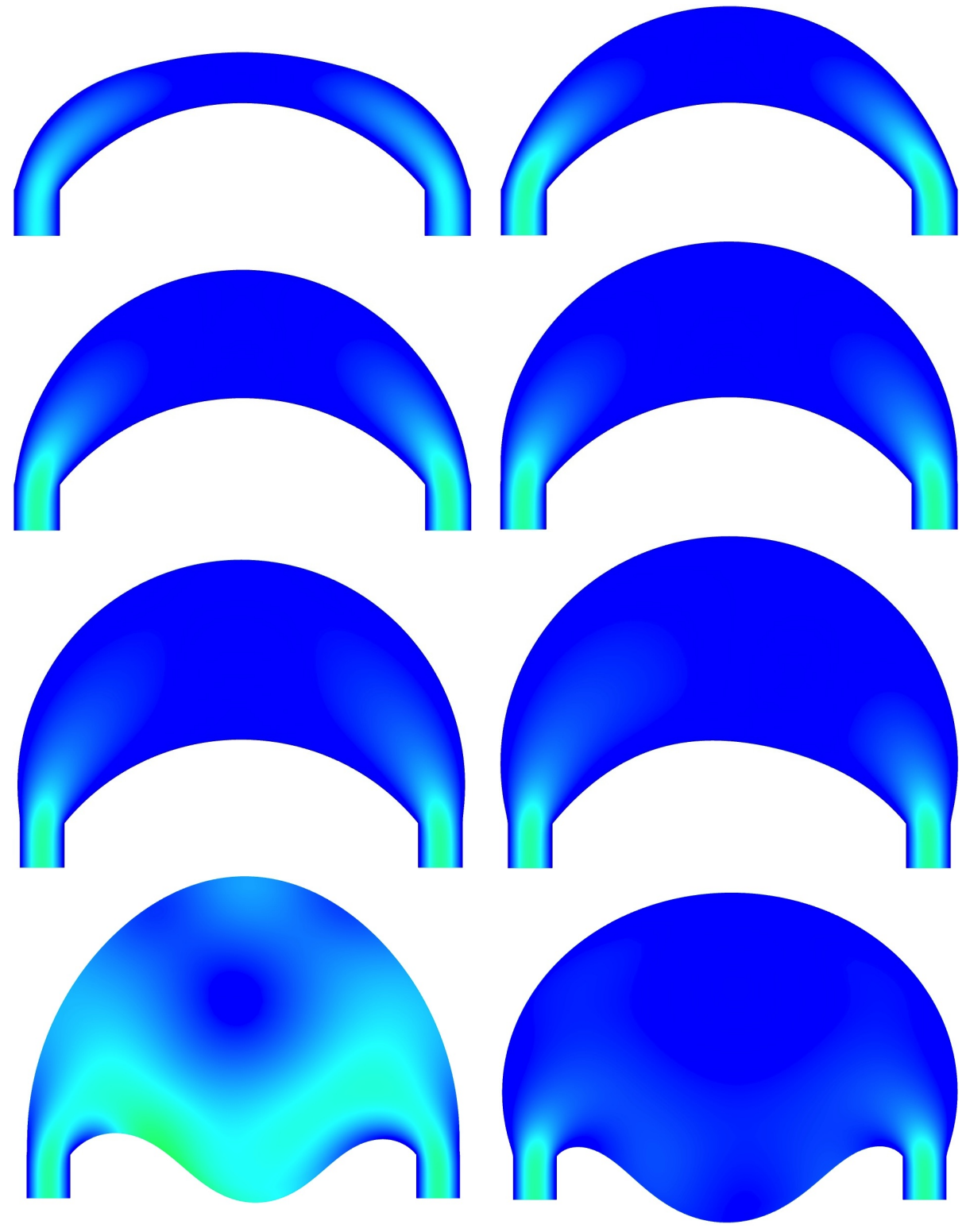

Figure 12: Snapshots of the fluid velocity $t=0.5,1,1.5,2,2.5,3,3.5,4$ (from left to right and top to bottom). Algorithm 4 with first-order extrapolation.

contrary, the variants with the first- and second-order extrapolations give practically the same result than the implicit scheme and predict the collapse of the bottom structure. 


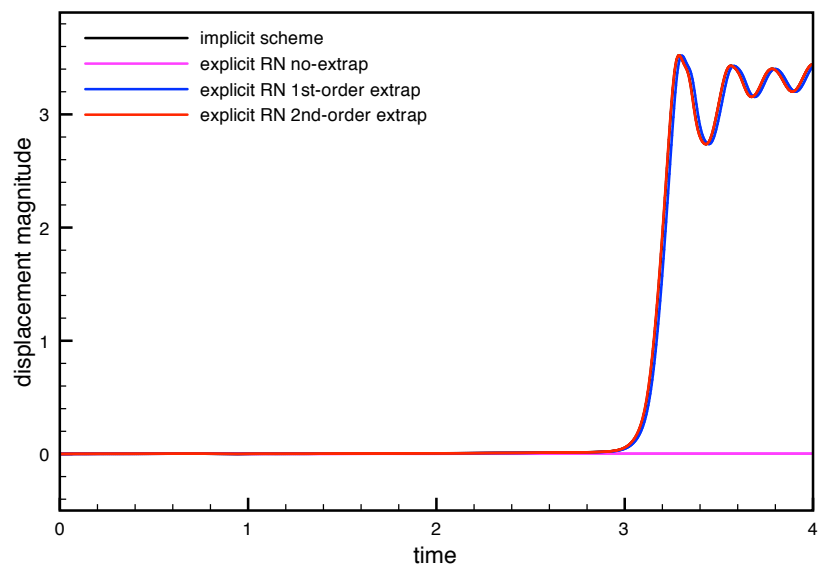

Figure 13: Comparison of the of the implicit and Robin-Neuman explicit coupling schemes: interface mid-point displacement magnitude of the bottom structure vs. time.

\section{Conclusion}

We have introduced and analyzed a class of explicit Robin-Neumann schemes for the coupling of a viscous incompressible fluid and a general thin-walled structure (including damping and non-linear behavior). The basis of these methods is the underlying Robin consistency of the interface coupling. This has motivated an explicit Robin interface condition for the fluid, which combines extrapolations of the solid viscoelastic contributions with an implicit treatment of the solid inertia. The former enables full fluid-solid splitting (i.e., explicit coupling), while the latter guarantees added-mass free stability (Theorem 1).

The schemes admit an intrinsic explicit Robin-Neumann formulation which makes them genuinely partitioned: only extrapolations of the solid velocity and interface fluid stress are necessary. The methods have been interpreted as single iterations, with appropriate initializations, of a (parameter free) Robin-Neumann iterative solution procedure of implicit coupling. New insights on the convergence of these procedures (Theorem 2) have been derived from this connection.

The error analysis (Theorem 3) has shown that the variants with extrapolation yield an optimal first time-accuracy in the energy-norm, while a sub-optimal convergence rate is expected without extrapolation. In summary, the key result is that the explicit Robin-Neumann scheme with first-order extrapolation simultaneously yields added-mass free unconditional stability and optimal (first-order) time accuracy.

A comprehensive list of numerical tests from the literature confirmed these findings, and indicate that the proposed methods provide a simple and robust approach to the explicit coupling of fluid-structure interaction problems, involving an incompressible fluid and a thin-walled structure.

Although not addressed herein, the techniques proposed can also be used to consider the case of thin-walled structures with constraints (e.g., inextensibility) enforced via Lagrange multipliers. An important setting, not covered by the present analysis, is the case of the coupling with thickwalled structures, for which the relation (5) is not valid anymore. A first attempt in this direction can be found in [25] (see also [24]), which generalizes the notion of interface Robin consistency using a mass lumping approximation in the solid. The resulting explicit coupling paradigm yields added-mass free stability (as in Theorem 1), but numerical evidence indicates that the 
optimality of the accuracy (as in Theorem 3) is not necessarily preserved. Further extensions of this work can explore various directions. We can address, for instance, the case of unfitted fluidsolid meshes and the development of second-order time-accurate schemes. Ongoing work focuses on the combination of the present explicit coupling paradigm with an overall fractional-step time-marching (fully decoupled state computation).

\section{A Proof of Theorem 1}

We proceed by extending the arguments reported in [21, Section 4]. Using (25), we take

$$
\left(\boldsymbol{v}_{h}, q_{h}\right)=\tau\left(\boldsymbol{u}_{h}^{n}, p_{h}^{n}\right), \quad \boldsymbol{w}_{h}=\tau \dot{\boldsymbol{d}}_{h}^{n}+\frac{\tau^{2}}{\rho^{\mathrm{s}} \epsilon}\left(\boldsymbol{L}_{h}^{\mathrm{e}}\left(\boldsymbol{d}_{h}^{n}-\boldsymbol{d}_{h}^{\star}\right)+\boldsymbol{L}_{h}^{\mathrm{v}}\left(\dot{\boldsymbol{d}}_{h}^{n}-\dot{\boldsymbol{d}}_{h}^{\star}\right)\right)
$$

as test functions in (26) for $n \geq r^{\star}+1$. Thus, since $\boldsymbol{f}^{\Gamma}=\mathbf{0}$, we get the following discrete energy equation

$$
\begin{aligned}
\frac{\rho^{\mathrm{f}}}{2}\left(\tau \partial_{\tau}\left\|\boldsymbol{u}_{h}^{n}\right\|_{0, \Omega}^{2}+\right. & \left.\left\|\boldsymbol{u}_{h}^{n}-\boldsymbol{u}_{h}^{n-1}\right\|_{0, \Omega}^{2}\right)+2 \mu \tau\left\|\varepsilon\left(\boldsymbol{u}_{h}^{n}\right)\right\|_{0, \Omega}^{2}+\tau\left|p_{h}^{n}\right|_{s_{h}}^{2} \\
+\frac{\rho^{\mathrm{s}} \epsilon}{2}\left(\tau \partial_{\tau}\left\|\dot{\boldsymbol{d}}_{h}^{n}\right\|_{0, \Sigma}^{2}+\right. & \left.\left\|\dot{\boldsymbol{d}}_{h}^{n}-\dot{\boldsymbol{d}}_{h}^{n-1}\right\|_{0, \Sigma}^{2}\right)+\frac{1}{2}\left(\tau \partial_{\tau}\left\|\boldsymbol{d}_{h}^{n}\right\|_{\mathrm{e}}^{2}+\left\|\boldsymbol{d}_{h}^{n}-\boldsymbol{d}_{h}^{n-1}\right\|_{\mathrm{e}}^{2}\right) \\
+\tau\left\|\dot{\boldsymbol{d}}_{h}^{n}\right\|_{\mathrm{v}}^{2}+ & \underbrace{\tau^{2}}_{T_{1}\left(\partial_{\tau} \dot{\boldsymbol{d}}_{h}^{n}, \boldsymbol{L}_{h}^{\mathrm{e}}\left(\boldsymbol{d}_{h}^{n}-\boldsymbol{d}_{h}^{\star}\right)+\boldsymbol{L}_{h}^{\mathrm{v}}\left(\dot{\boldsymbol{d}}_{h}^{n}-\dot{\boldsymbol{d}}_{h}^{\star}\right)\right)_{\Sigma}} \\
+ & \underbrace{\frac{\tau^{2}}{\rho^{\mathrm{s}} \epsilon}\left(\boldsymbol{L}_{h}^{\mathrm{e}} \boldsymbol{d}_{h}^{n}+\boldsymbol{L}_{h}^{\mathrm{v}} \dot{\boldsymbol{d}}_{h}^{n}, \boldsymbol{L}_{h}^{\mathrm{e}}\left(\boldsymbol{d}_{h}^{n}-\boldsymbol{d}_{h}^{\star}\right)+\boldsymbol{L}_{h}^{\mathrm{v}}\left(\dot{\boldsymbol{d}}_{h}^{n}-\dot{\boldsymbol{d}}_{h}^{\star}\right)\right)_{\Sigma}}_{T_{2}}=0
\end{aligned}
$$

for $n \geq r^{\star}+1$. To complete the proof, we only need to control the terms $T_{1}$ and $T_{2}$. We proceed by treating each case separately, depending on the extrapolation order $r^{\star}$.

(i) Without extrapolation $\left(r^{\star}=0\right)$. In this case, using Young's inequality, we have

$$
T_{1}+T_{2} \geq-\frac{\rho^{\mathrm{s}} \epsilon}{3}\left\|\dot{\boldsymbol{d}}_{h}^{n}-\dot{\boldsymbol{d}}_{h}^{n-1}\right\|_{0, \Sigma}^{2}+\frac{\tau^{2}}{4 \rho^{\mathrm{s}} \epsilon}\left\|\boldsymbol{L}_{h}^{\mathrm{e}} \boldsymbol{d}_{h}^{n}+\boldsymbol{L}_{h}^{\mathrm{v}} \dot{\boldsymbol{d}}_{h}^{n}\right\|_{0, \Sigma}^{2}
$$

for $n \geq 1$. Hence, the estimate (27) follows by inserting this expression into (44), applying Korn's inequality to the fluid viscous dissipation and summing over $m=1, \ldots, n$.

(ii) First-order extrapolation $\left(r^{\star}=1\right)$. In this case we have

$$
T_{1}=\frac{\tau^{2}}{2}\left(\tau \partial_{\tau}\left\|\dot{\boldsymbol{d}}_{h}^{n}\right\|_{\mathrm{e}}^{2}+\left\|\dot{\boldsymbol{d}}_{h}^{n}-\dot{\boldsymbol{d}}_{h}^{n-1}\right\|_{\mathrm{e}}^{2}\right)+\tau\left\|\dot{\boldsymbol{d}}_{h}^{n}-\dot{\boldsymbol{d}}_{h}^{n-1}\right\|_{\mathrm{v}}^{2}
$$

and

$$
T_{2}=\frac{\tau^{2}}{2 \rho^{\mathrm{s}} \epsilon}\left(\tau \partial_{\tau}\left\|\boldsymbol{L}_{h}^{\mathrm{e}} \boldsymbol{d}_{h}^{n}+\boldsymbol{L}_{h}^{\mathrm{v}} \dot{\boldsymbol{d}}_{h}^{n}\right\|_{0, \Sigma}^{2}+\left\|\boldsymbol{L}_{h}^{\mathrm{e}}\left(\boldsymbol{d}_{h}^{n}-\boldsymbol{d}_{h}^{n-1}\right)+\boldsymbol{L}_{h}^{\mathrm{v}}\left(\dot{\boldsymbol{d}}_{h}^{n}-\dot{\boldsymbol{d}}_{h}^{n-1}\right)\right\|_{0, \Sigma}^{2}\right)
$$

for $n \geq 2$. Hence, by inserting this expression into (44), use Korn's inequality and summing over $m=2, \ldots, n$ we get the estimate

$$
E_{h}^{n}+\sum_{m=2}^{n} D_{h}^{m} \lesssim E_{h}^{1}+\frac{\tau^{2}}{2}\left\|\dot{\boldsymbol{d}}_{h}^{1}\right\|_{\mathrm{e}}^{2}+\frac{\tau^{2}}{2 \rho^{\mathrm{s}} \epsilon}\left\|\boldsymbol{L}_{h}^{\mathrm{e}} \boldsymbol{d}_{h}^{1}+\boldsymbol{L}_{h}^{\mathrm{v}} \dot{\boldsymbol{d}}_{h}^{1}\right\|_{0, \Sigma}^{2}
$$

$\operatorname{RR} n^{\circ} 8224$ 
The last two terms, related to the initialization of the scheme (see Remark 4), can be bounded using (27) with $r^{\star}=0, n=1$ and the additional control given by (45). This yields the estimate (27) for $n \geq 2$.

(iii) Second-order extrapolation $\left(r^{\star}=2\right)$. In this case, we need the following estimates:

$$
\begin{aligned}
\left\|\boldsymbol{w}_{h}\right\|_{\mathrm{e}}^{2} & \leq \frac{\beta^{\mathrm{e}} C_{\mathrm{inv}}^{2}}{h^{2}}\left\|\boldsymbol{w}_{h}\right\|_{0, \Sigma}^{2}, \\
\left\|\boldsymbol{L}_{h}^{\mathrm{e}} \boldsymbol{w}_{h}\right\|_{\mathrm{e}} & \leq \frac{\beta^{\mathrm{e}} C_{\mathrm{inv}}^{2}}{h^{2}}\left\|\boldsymbol{w}_{h}\right\|_{\mathrm{e}} \\
\left\|\boldsymbol{L}_{h}^{\mathrm{e}} \boldsymbol{w}_{h}\right\|_{0, \Sigma} & \leq \frac{\left(\beta^{\mathrm{e}}\right)^{\frac{1}{2}} C_{\mathrm{inv}}}{h}\left\|\boldsymbol{w}_{h}\right\|_{\mathrm{e}} \\
\left\|\boldsymbol{L}_{h}^{\mathrm{e}} \boldsymbol{w}_{h}\right\|_{\mathrm{v}} & \leq \frac{\beta^{\mathrm{e}} C_{\mathrm{inv}}^{2}}{h^{2}}\left\|\boldsymbol{w}_{h}\right\|_{\mathrm{v}},
\end{aligned}
$$

for all $\boldsymbol{w}_{h} \in \boldsymbol{W}_{h}$ and with $C_{\mathrm{inv}}>0$ the constant of an inverse estimate. Details on the derivation of (48)-(50) are given in [21, Lemma 1], while (51) is a direct consequence of (3) and (48)-(50). For term $T_{1}$ in (44) we simply have

$$
T_{1}=\tau^{2}\left\|\dot{\boldsymbol{d}}_{h}^{n}-\dot{\boldsymbol{d}}_{h}^{n-1}\right\|_{\mathrm{e}}^{2}+\frac{\tau}{2}\left(\tau \partial_{\tau}\left\|\dot{\boldsymbol{d}}_{h}^{n}-\dot{\boldsymbol{d}}_{h}^{n-1}\right\|_{\mathrm{v}}^{2}+\left\|\dot{\boldsymbol{d}}_{h}^{n}-2 \dot{\boldsymbol{d}}_{h}^{n-1}+\dot{\boldsymbol{d}}_{h}^{n-2}\right\|_{\mathrm{v}}^{2}\right)
$$

Term $T_{2}$ is split as follows

$$
\begin{aligned}
T_{2}= & \underbrace{\frac{\tau^{2}}{\rho^{\mathrm{s}} \epsilon}\left(\boldsymbol{L}_{h}^{\mathrm{v}} \dot{\boldsymbol{d}}_{h}^{n}, \boldsymbol{L}_{h}^{\mathrm{e}}\left(\boldsymbol{d}_{h}^{n}-2 \boldsymbol{d}_{h}^{n-1}+\boldsymbol{d}_{h}^{n-2}\right)\right)_{\Sigma}}_{T_{2,1}} \\
& +\underbrace{\frac{\tau^{2}}{\rho^{\mathrm{s}} \epsilon}\left(\boldsymbol{L}_{h}^{\mathrm{e}} \boldsymbol{d}_{h}^{n}, \boldsymbol{L}_{h}^{\mathrm{e}}\left(\boldsymbol{d}_{h}^{n}-2 \boldsymbol{d}_{h}^{n-1}+\boldsymbol{d}_{h}^{n-2}\right)\right)_{\Sigma}}_{T_{2,2}} \\
& +\underbrace{\frac{\tau^{2}}{\rho^{\mathrm{s} \epsilon}}\left(\boldsymbol{L}_{h}^{\mathrm{e}} \boldsymbol{d}_{h}^{n}+\boldsymbol{L}_{h}^{\mathrm{v}} \dot{\boldsymbol{d}}_{h}^{n}, \boldsymbol{L}_{h}^{\mathrm{v}}\left(\dot{\boldsymbol{d}}_{h}^{n}-2 \dot{\boldsymbol{d}}_{h}^{n-1}+\dot{\boldsymbol{d}}_{h}^{n-2}\right)\right)_{\Sigma}}_{T_{2,3}}
\end{aligned}
$$

and we estimate each term separately. Using (3), for the first term we have

$$
\begin{aligned}
T_{2,1}= & \alpha \tau^{3}\left(\dot{\boldsymbol{d}}_{h}^{n}, \boldsymbol{L}_{h}^{\mathrm{e}}\left(\dot{\boldsymbol{d}}_{h}^{n}-\dot{\boldsymbol{d}}_{h}^{n-1}\right)\right)_{\Sigma}+\frac{\beta \tau^{3}}{\rho^{\mathrm{s} \epsilon}}\left(\boldsymbol{L}_{h}^{\mathrm{e}} \dot{\boldsymbol{d}}_{h}^{n}, \boldsymbol{L}_{h}^{\mathrm{e}}\left(\dot{\boldsymbol{d}}_{h}^{n}-\dot{\boldsymbol{d}}_{h}^{n-1}\right)\right)_{\Sigma} \\
= & \frac{\alpha \tau^{3}}{2}\left(\tau \partial_{\tau}\left\|\dot{\boldsymbol{d}}_{h}^{n}\right\|_{\mathrm{e}}^{2}+\left\|\dot{\boldsymbol{d}}_{h}^{n}-\dot{\boldsymbol{d}}_{h}^{n-1}\right\|_{\mathrm{e}}^{2}\right) \\
& +\frac{\beta \tau^{3}}{2 \rho^{\mathrm{s}} \epsilon}\left(\tau \partial_{\tau}\left\|\boldsymbol{L}_{h}^{\mathrm{e}} \dot{\boldsymbol{d}}_{h}^{n}\right\|_{0, \Sigma}^{2}+\left\|\boldsymbol{L}_{h}^{\mathrm{e}}\left(\dot{\boldsymbol{d}}_{h}^{n}-\dot{\boldsymbol{d}}_{h}^{n-1}\right)\right\|_{0, \Sigma}^{2}\right)
\end{aligned}
$$

The second term is treated as in [21, Page 38] using (48) and (49), which yields

$$
T_{2,2} \geq-\tau^{6} \frac{\left(\omega^{\mathrm{e}}\right)^{6}}{h^{6}}\left\|\boldsymbol{d}_{h}^{n}\right\|_{\mathrm{e}}^{2}-\frac{\rho^{\mathrm{s}} \epsilon}{4}\left\|\dot{\boldsymbol{d}}_{h}^{n}-\dot{\boldsymbol{d}}_{h}^{n-1}\right\|_{0, \Sigma}^{2}
$$

where we have used the notation $\omega^{\mathrm{e}} \stackrel{\text { def }}{=} C_{\mathrm{inv}} \sqrt{\beta^{\mathrm{e}} /\left(\rho^{\mathrm{s}} \epsilon\right)}$. For the last term, we first use relation 
(3) to obtain

$$
\begin{aligned}
T_{2,3} \geq & -\frac{\tau^{2}}{\rho^{\mathrm{s}} \epsilon}\left\|\boldsymbol{L}_{h}^{\mathrm{e}} \boldsymbol{d}_{h}^{n}+\boldsymbol{L}_{h}^{\mathrm{v}} \dot{\boldsymbol{d}}_{h}^{n}\right\|_{\mathrm{v}}\left\|\dot{\boldsymbol{d}}_{h}^{n}-2 \dot{\boldsymbol{d}}_{h}^{n-1}+\dot{\boldsymbol{d}}_{h}^{n-2}\right\|_{\mathrm{v}} \\
\geq & -\frac{2 \tau^{3}}{\left(\rho^{\mathrm{s}} \epsilon\right)^{2}}\left\|\boldsymbol{L}_{h}^{\mathrm{e}} \boldsymbol{d}_{h}^{n}\right\|_{\mathrm{v}}^{2}-\frac{2 \tau^{3}}{\left(\rho^{\mathrm{s}} \epsilon\right)^{2}}\left\|\boldsymbol{L}_{h}^{\mathrm{v}} \dot{\boldsymbol{d}}_{h}^{n}\right\|_{\mathrm{v}}^{2}-\frac{\tau}{4}\left\|\dot{\boldsymbol{d}}_{h}^{n}-2 \dot{\boldsymbol{d}}_{h}^{n-1}+\dot{\boldsymbol{d}}_{h}^{n-2}\right\|_{\mathrm{v}}^{2} \\
\geq & -\frac{2 \tau^{3}}{\rho^{\mathrm{s}} \epsilon}\left(\alpha\left\|\boldsymbol{L}_{h}^{\mathrm{e}} \boldsymbol{d}_{h}^{n}\right\|_{0, \Sigma}^{2}+\frac{\beta}{\rho^{\mathrm{s}} \epsilon}\left\|\boldsymbol{L}_{h}^{\mathrm{e}} \boldsymbol{d}_{h}^{n}\right\|_{\mathrm{e}}^{2}\right) \\
& -4 \tau^{3}\left(\alpha^{2}\left\|\dot{\boldsymbol{d}}_{h}^{n}\right\|_{\mathrm{v}}^{2}+\frac{\beta^{2}}{\left(\rho^{\mathrm{s}} \epsilon\right)^{2}}\left\|\boldsymbol{L}_{h}^{\mathrm{e}} \dot{\boldsymbol{d}}_{h}^{n}\right\|_{\mathrm{v}}^{2}\right)-\frac{\tau}{4}\left\|\dot{\boldsymbol{d}}_{h}^{n}-2 \dot{\boldsymbol{d}}_{h}^{n-1}+\dot{\boldsymbol{d}}_{h}^{n-2}\right\|_{\mathrm{v}}^{2} .
\end{aligned}
$$

Then, using (49)-(51), we get

$$
\begin{aligned}
T_{2,3} \geq & -\frac{2 \tau^{3}\left(\omega^{\mathrm{e}}\right)^{2}}{h^{2}}\left(\alpha+\frac{\beta^{2}\left(\omega^{\mathrm{e}}\right)^{2}}{h^{2}}\right)\left\|\boldsymbol{d}_{h}^{n}\right\|_{\mathrm{e}}^{2} \\
& -4 \tau^{3}\left(\alpha^{2}+\frac{\beta^{2}\left(\omega^{\mathrm{e}}\right)^{4}}{h^{4}}\right)\left\|\dot{\boldsymbol{d}}_{h}^{n}\right\|_{\mathrm{v}}^{2}-\frac{\tau}{4}\left\|\dot{\boldsymbol{d}}_{h}^{n}-2 \dot{\boldsymbol{d}}_{h}^{n-1}+\dot{\boldsymbol{d}}_{h}^{n-2}\right\|_{\mathrm{v}}^{2} .
\end{aligned}
$$

Finally, by collecting the estimates (55)-(56) and using the conditions $(29)_{1,2}$, we obtain

$$
\begin{aligned}
T_{2,2}+T_{2,3} \geq & -\tau \frac{\gamma}{2}\left\|\boldsymbol{d}_{h}^{n}\right\|_{\mathrm{e}}^{2}-\tau \delta\left\|\dot{\boldsymbol{d}}_{h}^{n}\right\|_{\mathrm{v}}^{2}-\frac{\rho^{\mathrm{s}} \epsilon}{4}\left\|\dot{\boldsymbol{d}}_{h}^{n}-\dot{\boldsymbol{d}}_{h}^{n-1}\right\|_{0, \Sigma}^{2} \\
& -\frac{\tau}{4}\left\|\dot{\boldsymbol{d}}_{h}^{n}-2 \dot{\boldsymbol{d}}_{h}^{n-1}+\dot{\boldsymbol{d}}_{h}^{n-2}\right\|_{\mathrm{v}}^{2}
\end{aligned}
$$

We now proceed by inserting (52), (54) and (57) into (44), using Korn's inequality and summing over $m=3, \ldots, n$. The last three negative terms of (57) are controlled by the physical and numerical dissipations provided by (44) and (52), while the first is handled via Gronwall's Lemma (see, e.g., [36, Lemma 5.1]) under the condition $(29)_{3}$. This yields the bound

$$
\begin{gathered}
E_{h}^{n}+\sum_{m=3}^{n} D_{h}^{m} \lesssim \exp \left(\frac{t_{n} \gamma}{1-\tau \gamma}\right)\left(E_{h}^{2}+\tau\left\|\dot{\boldsymbol{d}}_{h}^{2}-\dot{\boldsymbol{d}}_{h}^{1}\right\|_{\mathrm{v}}^{2}+\alpha \tau^{3}\left\|\dot{\boldsymbol{d}}_{h}^{2}\right\|_{\mathrm{e}}^{2}\right. \\
\left.+\frac{\beta \tau^{3}}{\rho^{\mathrm{s}} \epsilon}\left\|\boldsymbol{L}_{h}^{\mathrm{e}} \dot{\boldsymbol{d}}_{h}^{2}\right\|_{0, \Sigma}^{2}\right)
\end{gathered}
$$

The estimate (28) then follows by using the energy estimate (27) with $r^{\star}=1$ and $n=2$, the additional control provided by (46) and (47), and the stability condition (29).

\section{B Proof of Theorem 2}

For $k \geq 1$, we consider $(17)_{1}$ at the $(k-1)$-th iteration and add the resulting expression to $(16)_{5}$. This yields

$$
\frac{\rho^{\mathrm{s}} \epsilon}{\tau}\left(\boldsymbol{u}_{k}-\dot{\boldsymbol{d}}^{n-1}\right)+\boldsymbol{L}^{\mathrm{e}} \boldsymbol{d}_{k-1}+\boldsymbol{L}^{\mathrm{v}} \dot{\boldsymbol{d}}_{k-1}=-\boldsymbol{\sigma}\left(\boldsymbol{u}_{k}, p_{k}\right) \boldsymbol{n} \quad \text { on } \quad \Sigma .
$$

Hence, by combining this relation with $(17)_{1}$ we get

$$
\boldsymbol{u}_{k}=\dot{\boldsymbol{d}}_{k}+\frac{\tau}{\rho^{\mathrm{s}} \epsilon}\left(\boldsymbol{L}^{\mathrm{e}}\left(\boldsymbol{d}_{k}-\boldsymbol{d}_{k-1}\right)+\boldsymbol{L}^{\mathrm{v}}\left(\dot{\boldsymbol{d}}_{k}-\dot{\boldsymbol{d}}_{k-1}\right)\right) \quad \text { on } \quad \Sigma .
$$

$\mathrm{RR} \mathrm{n}^{\circ} 8224$ 
It should be noted that $\boldsymbol{d}_{0}$ is defined through $(17)_{1}$, with $k=0$, in terms of $\dot{\boldsymbol{d}}_{0}$ and $\boldsymbol{\sigma}\left(\boldsymbol{u}_{0}, p_{0}\right) \boldsymbol{n}$. We now introduce the following errors between the $k$-th iteration of Algorithm 2 and the $n$-th step of (14)-(15):

$$
\boldsymbol{e}_{k}^{\boldsymbol{u}} \stackrel{\text { def }}{=} \boldsymbol{u}_{k}-\boldsymbol{u}^{n}, \quad e_{k}^{p} \stackrel{\text { def }}{=} p_{k}-p^{n}, \quad \boldsymbol{e}_{k}^{\dot{\boldsymbol{d}}} \stackrel{\text { def }}{=} \dot{\boldsymbol{d}}_{k}-\dot{\boldsymbol{d}}^{n}, \quad \boldsymbol{e}_{k}^{\boldsymbol{d}} \stackrel{\text { def }}{=} \boldsymbol{d}_{k}-\boldsymbol{d}^{n} .
$$

By subtracting (14)-(15) from $(16)_{1-4},(17)$ and (58), we get the following system of equations for the errors:

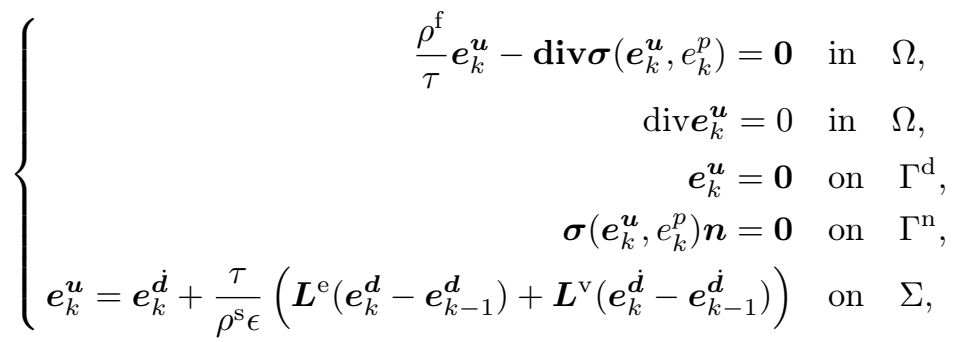

$$
\begin{aligned}
& \left\{\begin{array}{l}
\frac{\rho^{\mathrm{s}} \epsilon}{\tau} \boldsymbol{e}^{\dot{\boldsymbol{d}}_{k}}+\boldsymbol{L}^{\mathrm{e}} \boldsymbol{e}_{k}^{\boldsymbol{d}}+\boldsymbol{L}^{\mathrm{v}} \boldsymbol{e}_{k}^{\dot{\boldsymbol{d}}}=-\boldsymbol{\sigma}\left(\boldsymbol{e}_{k}^{\boldsymbol{u}}, e_{k}^{p}\right) \boldsymbol{n} \text { on } \Sigma, \\
\boldsymbol{e}_{k}^{\dot{\boldsymbol{d}}}=\frac{1}{\tau} \boldsymbol{e}_{k}^{\boldsymbol{d}} .
\end{array}\right.
\end{aligned}
$$

We now proceed similarly to the proof of Theorem 1 with the first-order extrapolation. We first multiply $(60)_{1}$ by $\tau \boldsymbol{e}_{k}^{\boldsymbol{u}}$ and integrate by parts in $\Omega$. Then we multiply $(61)_{2}$ by $\left.\tau \boldsymbol{e}_{k}^{\boldsymbol{u}}\right|_{\Sigma}$ and integrate the resulting expression over $\Sigma$. By adding these two expressions and using $(61)_{1,3}$ we get

$$
\begin{aligned}
\rho^{\mathrm{f}}\left\|\boldsymbol{e}_{k}^{\boldsymbol{u}}\right\|_{0, \Omega}^{2}+2 \mu \tau\left\|\boldsymbol{\varepsilon}\left(\boldsymbol{e}_{k}^{\boldsymbol{u}}\right)\right\|_{0, \Omega}^{2} & +\rho^{\mathrm{s}} \epsilon\left\|\boldsymbol{e}^{\dot{\boldsymbol{d}}_{k}}\right\|_{0, \Sigma}^{2}+\left\|\boldsymbol{e}^{\boldsymbol{d}_{k}}\right\|_{\mathrm{e}}^{2}+\tau\left\|\boldsymbol{e}^{\dot{\boldsymbol{d}}_{k}}\right\|_{\mathrm{v}}^{2} \\
+ & \underbrace{\tau\left(\boldsymbol{e}_{k}^{\boldsymbol{d}}, \boldsymbol{L}^{\mathrm{e}}\left(\boldsymbol{e}_{k}^{\boldsymbol{d}}-\boldsymbol{e}_{k-1}^{\boldsymbol{d}}\right)+\boldsymbol{L}^{\mathrm{v}}\left(\boldsymbol{e}_{k}^{\dot{\boldsymbol{d}}}-\boldsymbol{e}_{k-1}^{\dot{\boldsymbol{d}}}\right)\right)_{\Sigma}}_{T_{1}} \\
& +\underbrace{\frac{\tau^{2}}{\rho^{\mathrm{s}} \epsilon}\left(\boldsymbol{L}^{\mathrm{e}} \boldsymbol{e}_{k}^{\boldsymbol{d}}+\boldsymbol{L}^{\mathrm{v}} \boldsymbol{e}_{k}^{\dot{\boldsymbol{d}}}, \boldsymbol{L}^{\mathrm{e}}\left(\boldsymbol{e}_{k}^{\boldsymbol{d}}-\boldsymbol{e}_{k-1}^{\boldsymbol{d}}\right)+\boldsymbol{L}^{\mathrm{v}}\left(\boldsymbol{e}_{k}^{\dot{\boldsymbol{d}}}-\boldsymbol{e}_{k-1}^{\dot{\boldsymbol{d}}}\right)\right)_{\Sigma}}_{T_{2}}=0
\end{aligned}
$$

where it only remains to estimate the terms $T_{1}$ and $T_{2}$. For the first term, using $(61)_{3}$, we have

$$
T_{1}=\frac{1}{2}\left(\left\|\boldsymbol{e}_{k}^{\boldsymbol{d}}\right\|_{\mathrm{e}}^{2}-\left\|\boldsymbol{e}_{k-1}^{\boldsymbol{d}}\right\|_{\mathrm{e}}^{2}+\left\|\boldsymbol{e}_{k}^{\boldsymbol{d}}-\boldsymbol{e}_{k-1}^{\boldsymbol{d}}\right\|_{\mathrm{e}}^{2}\right)+\frac{\tau}{2}\left(\left\|\boldsymbol{e}_{k}^{\dot{d}}\right\|_{\mathrm{v}}^{2}-\left\|\boldsymbol{e}_{k-1}^{\dot{d}}\right\|_{\mathrm{v}}^{2}+\left\|\boldsymbol{e}_{k}^{\dot{d}}-\boldsymbol{e}_{k-1}^{\dot{d}}\right\|_{\mathrm{v}}^{2}\right)
$$

while, for the second, we get

$$
\begin{aligned}
T_{2}= & \frac{\tau^{2}}{2 \rho^{\mathrm{s}} \epsilon}\left(\left\|\boldsymbol{L}^{\mathrm{e}} \boldsymbol{e}_{k}^{\boldsymbol{d}}+\boldsymbol{L}^{\mathrm{v}} \boldsymbol{e}_{k}^{\dot{\boldsymbol{d}}}\right\|_{0, \Sigma}^{2}-\left\|\boldsymbol{L}^{\mathrm{e}} \boldsymbol{e}_{k-1}^{\boldsymbol{d}}+\boldsymbol{L}^{\mathrm{v}} \boldsymbol{e}_{k-1}^{\dot{\boldsymbol{d}}}\right\|_{0, \Sigma}^{2}\right) \\
& +\frac{\tau^{2}}{2 \rho^{\mathrm{s}} \epsilon}\left\|\boldsymbol{L}^{\mathrm{e}}\left(\boldsymbol{e}_{k}^{\boldsymbol{d}}-\boldsymbol{e}_{k-1}^{\boldsymbol{d}}\right)+\boldsymbol{L}^{\mathrm{v}}\left(\boldsymbol{e}_{k}^{\dot{\boldsymbol{d}}}-\boldsymbol{e}_{k-1}^{\dot{\boldsymbol{d}}}\right)\right\|_{0, \Sigma}^{2}
\end{aligned}
$$

The estimate (30) follows by inserting these last two identities into (62) and after summation over $k=1, \ldots, \infty$. 


\section{Proof of Theorem 3}

We proceed by extending the arguments reported in [21, Section 5]. The error estimate (37) is based on the following decomposition of the error

$$
\begin{aligned}
& \boldsymbol{u}\left(t_{n}\right)-\boldsymbol{u}_{h}^{n}=\underbrace{\boldsymbol{u}\left(t_{n}\right)-\boldsymbol{P}_{h} \boldsymbol{u}\left(t_{n}\right)}_{\boldsymbol{\theta}_{\pi}^{n}}+\underbrace{\boldsymbol{P}_{h} \boldsymbol{u}\left(t_{n}\right)-\boldsymbol{u}_{h}^{n}}_{\boldsymbol{\theta}_{h}^{n}} \\
& p\left(t_{n}\right)-p_{h}^{n}=\underbrace{p\left(t_{n}\right)-\Pi_{h} p\left(t_{n}\right)}_{y_{\pi}^{n}}+\underbrace{\Pi_{h} p\left(t_{n}\right)-p_{h}^{n}}_{y_{h}^{n}}, \\
& \boldsymbol{d}\left(t_{n}\right)-\boldsymbol{d}_{h}^{n}=\underbrace{\boldsymbol{d}\left(t_{n}\right)-\boldsymbol{\pi}_{h}^{\mathrm{e}} \boldsymbol{d}\left(t_{n}\right)}_{\boldsymbol{\xi}_{\pi}^{n}}+\underbrace{\boldsymbol{\pi}_{h}^{\mathrm{e}} \boldsymbol{d}\left(t_{n}\right)-\boldsymbol{d}_{h}^{n}}_{\boldsymbol{\xi}_{h}^{n}}, \\
& \dot{\boldsymbol{d}}\left(t_{n}\right)-\dot{\boldsymbol{d}}_{h}^{n}=\underbrace{\dot{\boldsymbol{d}}\left(t_{n}\right)-\boldsymbol{\pi}_{h}^{\mathrm{v}} \dot{\boldsymbol{d}}^{n}}_{\dot{\boldsymbol{\xi}}_{\pi}^{n}}+\underbrace{\boldsymbol{\pi}_{h}^{\mathrm{v}} \dot{\boldsymbol{d}}\left(t_{n}\right)-\dot{\boldsymbol{d}}_{h}^{n}}_{\dot{\boldsymbol{\xi}}_{h}^{n}},
\end{aligned}
$$

where the projection operators $\boldsymbol{P}_{h}, \Pi_{h}, \boldsymbol{\pi}_{h}^{\mathrm{e}}$ and $\boldsymbol{\pi}_{h}^{\mathrm{v}}$ are defined in Section 4.6. Hence, owing to (32)-(34), it only remains to estimate the discrete contributions $\boldsymbol{\theta}_{h}^{n}, y_{h}^{n}, \boldsymbol{\xi}_{h}^{n}$ and $\dot{\boldsymbol{\xi}}_{h}^{n}$. An a priori bound for these terms is stated in Lemma 4 below, with the energy-norm of the discrete error being defined by

$$
\begin{gathered}
\mathcal{Z}_{h}^{n} \stackrel{\text { def }}{=}\left(\rho^{\mathrm{f}}\right)^{\frac{1}{2}}\left\|\boldsymbol{\theta}_{h}^{n}\right\|_{0, \Omega}+\left(\sum_{m=r^{\star}+1}^{n} \tau\left\|\boldsymbol{\theta}_{h}^{m}\right\|_{\boldsymbol{V}}^{2}\right)^{\frac{1}{2}}+\left(\sum_{m=r^{\star}+1}^{n} \tau\left|y_{h}^{m}\right|_{s_{h}}^{2}\right)^{\frac{1}{2}} \\
+\left(\rho^{\mathrm{s}} \epsilon\right)^{\frac{1}{2}}\left\|\dot{\boldsymbol{\xi}}_{h}^{n}\right\|_{0, \Sigma}+\left\|\boldsymbol{\xi}_{h}^{n}\right\|_{\mathrm{e}}+\left(\sum_{m=r^{\star}+1}^{n} \tau\left\|\dot{\boldsymbol{\xi}}_{h}^{m}\right\|_{\mathrm{v}}^{2}\right)^{\frac{1}{2}}
\end{gathered}
$$

for $n \geq r^{\star}+1$.

Lemma 4 Under the assumptions of Theorem 3, the following error estimates hold:

- Without extrapolation $\left(r^{\star}=0\right)$ :

$$
\mathcal{Z}_{h}^{n} \lesssim c_{1} h^{k}+c_{2} h^{\tilde{l}}+c_{3} \tau+c_{4} \tau^{\frac{1}{2}}\left(\frac{T}{\rho^{\mathrm{s}} \epsilon}\right)^{\frac{1}{2}}\left\|\boldsymbol{L}^{\mathrm{e}} \boldsymbol{d}+\boldsymbol{L}^{\mathrm{v}} \dot{\boldsymbol{d}}\right\|_{L^{\infty}\left(0, T ; L^{2}(\Sigma)\right)}
$$

for $n \geq 1$.

- First-order extrapolation $\left(r^{\star}=1\right)$ :

$$
\mathcal{Z}_{h}^{n} \lesssim c_{1} h^{k}+c_{2} h^{\tilde{l}}+c_{3} \tau+c_{4} \tau\left(\frac{T}{\rho^{\mathrm{s}} \epsilon}\right)^{\frac{1}{2}}\left\|\partial_{t}\left(\boldsymbol{L}^{\mathrm{e}} \boldsymbol{d}+\boldsymbol{L}^{\mathrm{v}} \dot{\boldsymbol{d}}\right)\right\|_{L^{2}\left(0, T ; L^{2}(\Sigma)\right)}
$$

for $n \geq 2$.

- Second-order extrapolation $\left(r^{\star}=2\right)$ :

$$
\mathcal{Z}_{h}^{n} \lesssim c_{5} h^{k}+c_{6} h^{\tilde{l}}+c_{7} \tau+c_{8} \tau^{2}\left(\frac{T}{\rho^{\mathrm{s}} \epsilon}\right)^{\frac{1}{2}}\left\|\partial_{t t}\left(\boldsymbol{L}^{\mathrm{e}} \boldsymbol{d}+\boldsymbol{L}^{\mathrm{v}} \dot{\boldsymbol{d}}\right)\right\|_{L^{2}\left(0, T ; L^{2}(\Sigma)\right)}
$$

for $n \geq 3$.

$\mathrm{RR} \mathrm{n}^{\circ} 8224$ 
Here, the symbols $\left\{c_{i}\right\}_{i=1}^{8}$ denote positive constants independent of $h$ and $\tau$, but which depend on the physical parameters and on the regularity of $(\boldsymbol{u}, p, \boldsymbol{d}, \dot{\boldsymbol{d}})$.

Proof 4 We first recall the following properties of the discrete solid operators $\boldsymbol{L}_{h}^{\mathrm{e}}$ and $\boldsymbol{L}_{h}^{\mathrm{v}}$ (see, e.g., [21, Lemma 1]), which will be used below:

$$
\boldsymbol{L}_{h}^{\mathrm{e}} \boldsymbol{\pi}_{h}^{\mathrm{e}}=\boldsymbol{L}_{h}^{\mathrm{e}}, \quad \boldsymbol{L}_{h}^{\mathrm{v}} \boldsymbol{\pi}_{h}^{\mathrm{v}}=\boldsymbol{L}_{h}^{\mathrm{v}}
$$

and

$$
\left\|\boldsymbol{L}_{h}^{\mathrm{e}} \boldsymbol{w}\right\|_{0, \Sigma} \leq\left\|\boldsymbol{L}^{\mathrm{e}} \boldsymbol{w}\right\|_{0, \Sigma}, \quad\left\|\boldsymbol{L}_{h}^{\mathrm{v}} \dot{\boldsymbol{w}}\right\|_{0, \Sigma} \leq\left\|\boldsymbol{L}^{\mathrm{v}} \dot{\boldsymbol{w}}\right\|_{0, \Sigma}
$$

for all $\boldsymbol{w} \in \boldsymbol{D}^{\mathrm{e}}$ and $\boldsymbol{w} \in \boldsymbol{D}^{\mathrm{v}}$

We consider the continuous problem (19) at time $t=t_{n}$ with $(\boldsymbol{v}, q, \boldsymbol{w})=\left(\boldsymbol{v}_{h}, q_{h}, \boldsymbol{w}_{h}\right) \in$ $\boldsymbol{V}_{h} \times Q_{h} \times \boldsymbol{W}_{h}$, and subtract (26) from the resulting expression. This yields the following modified Galerkin orthogonality:

$$
\begin{array}{r}
\rho^{\mathrm{f}}\left(\partial_{\tau}\left(\boldsymbol{u}^{n}-\boldsymbol{u}_{h}^{n}\right), \boldsymbol{v}_{h}\right)+a\left(\boldsymbol{u}\left(t_{n}\right)-\boldsymbol{u}_{h}^{n}, \boldsymbol{v}_{h}\right)+b\left(p\left(t_{n}\right)-p_{h}^{n}, \boldsymbol{v}_{h}\right)-b\left(q_{h}, \boldsymbol{u}\left(t_{n}\right)-\boldsymbol{u}_{h}^{n}\right) \\
+\rho^{\mathrm{s}} \epsilon\left(\partial_{\tau}\left(\dot{\boldsymbol{d}}\left(t_{n}\right)-\dot{\boldsymbol{d}}_{h}^{n}\right), \boldsymbol{w}_{h}\right)_{\Sigma}+a^{\mathrm{e}}\left(\boldsymbol{d}\left(t_{n}\right)-\boldsymbol{d}_{h}^{n}, \boldsymbol{w}_{h}\right)+a^{\mathrm{v}}\left(\dot{\boldsymbol{d}}\left(t_{n}\right)-\dot{\boldsymbol{d}}_{h}^{n}, \boldsymbol{w}_{h}\right) \\
=-\rho^{\mathrm{f}}\left(\left(\partial_{t}-\partial_{\tau}\right) \boldsymbol{u}\left(t_{n}\right), \boldsymbol{v}_{h}\right)-\rho^{\mathrm{s}} \epsilon\left(\left(\partial_{t}-\partial_{\tau}\right) \dot{\boldsymbol{d}}\left(t_{n}\right), \boldsymbol{w}_{h}\right)_{\Sigma}+s_{h}\left(p_{h}^{n}, q_{h}\right)
\end{array}
$$

for all $\left(\boldsymbol{v}_{h}, q_{h}, \boldsymbol{w}_{h}\right) \in \boldsymbol{V}_{h} \times Q_{h} \times \boldsymbol{W}_{h}$ with $\left.\boldsymbol{v}_{h}\right|_{\Sigma}=\boldsymbol{w}_{h}$. Hence, using (63), we infer the following equation for the discrete errors $\boldsymbol{\theta}_{h}^{n}, y_{h}^{n}, \boldsymbol{\xi}_{h}^{n}$ and $\dot{\boldsymbol{\xi}}_{h}^{n}$ :

$$
\begin{aligned}
\rho^{\mathrm{f}} & \left(\partial_{\tau} \boldsymbol{\theta}_{h}^{n}, \boldsymbol{v}_{h}\right)+a\left(\boldsymbol{\theta}_{h}^{n}, \boldsymbol{v}_{h}\right)+b\left(y_{h}^{n}, \boldsymbol{v}_{h}\right)-b\left(q_{h}, \boldsymbol{\theta}_{h}^{n}\right)+s_{h}\left(y_{h}^{n}, q_{h}\right) \\
& +\rho^{\mathrm{s}} \epsilon\left(\partial_{\tau} \dot{\boldsymbol{\xi}}_{h}^{n}, \boldsymbol{v}_{h}\right)_{\Sigma}+a^{\mathrm{e}}\left(\boldsymbol{\xi}_{h}^{n}, \boldsymbol{v}_{h}\right)+a^{\mathrm{v}}\left(\dot{\boldsymbol{\xi}}_{h}^{n}, \boldsymbol{v}_{h}\right) \\
= & \underbrace{-\rho^{\mathrm{f}}\left(\left(\partial_{t}-\partial_{\tau}\right) \boldsymbol{u}\left(t_{n}\right), \boldsymbol{v}_{h}\right)-\rho^{\mathrm{f}}\left(\partial_{\tau} \boldsymbol{\theta}_{\pi}^{n}, \boldsymbol{v}_{h}\right)}_{T_{1}\left(\boldsymbol{v}_{h}\right)} \\
& -\underbrace{\left.\rho_{\mathrm{s}}^{\mathrm{s}} \epsilon\left(\partial_{t}-\partial_{\tau}\right) \dot{\boldsymbol{d}}\left(t_{n}\right), \boldsymbol{v}_{h}\right)_{\Sigma}-\rho^{\mathrm{s}} \epsilon\left(\partial_{\tau} \dot{\boldsymbol{\xi}}_{\pi}^{n}, \boldsymbol{v}_{h}\right)_{\Sigma}}_{T_{2}\left(\boldsymbol{v}_{h}\right)} \\
& +\underbrace{s_{h}\left(\Pi_{h} p^{n}, q_{h}\right)-a\left(\boldsymbol{\theta}_{\pi}^{n}, \boldsymbol{v}_{h}\right)-b\left(y_{\pi}^{n}, \boldsymbol{v}_{h}\right)+b\left(q_{h}, \boldsymbol{\theta}_{\pi}^{n}\right)}_{T_{3}\left(\boldsymbol{v}_{h}, q_{h}\right)} \\
& -\underbrace{a^{\mathrm{e}\left(\boldsymbol{\xi}_{\pi}^{n}, \boldsymbol{v}_{h}\right)}-\underbrace{a^{\mathrm{v}}\left(\dot{\boldsymbol{\xi}}_{\pi}^{n}, \boldsymbol{v}_{h}\right)}_{=0}}_{=0}
\end{aligned}
$$

for all $\left(\boldsymbol{v}_{h}, q_{h}\right) \in \boldsymbol{V}_{h} \times Q_{h}$ with $\left.\boldsymbol{v}_{h}\right|_{\Sigma} \in \boldsymbol{W}_{h}$ and $n \geq r^{\star}+1$. It should be noted that last two terms vanish due to definition (31) of the solid projection operators.

Moreover, by combining (25) and (67) with (63), we have

$$
\begin{aligned}
\left.\boldsymbol{\theta}_{h}^{n}\right|_{\Sigma}= & \dot{\boldsymbol{\xi}}_{h}^{n}+\frac{\tau}{\rho^{\mathrm{s}} \epsilon}\left(\boldsymbol{L}_{h}^{\mathrm{e}}\left(\boldsymbol{\xi}_{h}^{n}-\boldsymbol{\xi}_{h}^{\star}\right)+\boldsymbol{L}_{h}^{\mathrm{v}}\left(\dot{\boldsymbol{\xi}}_{h}^{n}-\dot{\boldsymbol{\xi}}_{h}^{\star}\right)\right) \\
& -\frac{\tau}{\rho^{\mathrm{s}} \epsilon}\left(\boldsymbol{L}_{h}^{\mathrm{e}}\left(\boldsymbol{d}\left(t_{n}\right)-\boldsymbol{d}^{\star}\right)+\boldsymbol{L}_{h}^{\mathrm{v}}\left(\dot{\boldsymbol{d}}\left(t_{n}\right)-\dot{\boldsymbol{d}}^{\star}\right)\right)
\end{aligned}
$$

for $n \geq r^{\star}+1$, with the natural notations $\boldsymbol{\xi}_{h}^{\star} \stackrel{\text { def }}{=} \boldsymbol{\pi}_{h}^{\mathrm{e}} \boldsymbol{d}^{\star}-\boldsymbol{d}_{h}^{\star}, \dot{\boldsymbol{\xi}}_{h}^{\star} \stackrel{\text { def }}{=} \boldsymbol{\pi}_{h}^{\vee} \dot{\boldsymbol{d}}^{\star}-\dot{\boldsymbol{d}}_{h}^{\star}$ and

$$
\left(\boldsymbol{d}^{\star}, \dot{\boldsymbol{d}}^{\star}\right)= \begin{cases}\mathbf{0} & \text { if } r^{\star}=0, \\ \left(\boldsymbol{d}\left(t_{n-1}\right), \dot{\boldsymbol{d}}\left(t_{n-1}\right)\right) & \text { if } r^{\star}=1, \\ \left(2 \boldsymbol{d}\left(t_{n-1}\right)-\boldsymbol{d}\left(t_{n-2}\right), 2 \dot{\boldsymbol{d}}\left(t_{n-1}\right)-\dot{\boldsymbol{d}}\left(t_{n-2}\right)\right) & \text { if } r^{\star}=2 .\end{cases}
$$


Similarly, from $(21)_{1}$ and (63), it follows that

$$
\dot{\boldsymbol{\xi}}_{h}^{n}=\partial_{\tau} \boldsymbol{\xi}_{h}^{n}+\underbrace{\boldsymbol{\pi}_{h}^{\mathrm{v}} \dot{\boldsymbol{d}}\left(t_{n}\right)-\boldsymbol{\pi}_{h}^{\mathrm{e}} \partial_{\tau} \boldsymbol{d}\left(t_{n}\right)}_{\boldsymbol{z}_{h}^{n}} .
$$

In order to control the perturbation introduced by the last term (72), we shall make use of the following estimates

$$
\begin{aligned}
\left\|\boldsymbol{z}_{h}^{n}\right\|_{\mathrm{e}} \lesssim & \left(\beta^{\mathrm{e}}\right)^{\frac{1}{2}}\left(h^{k}\left\|\boldsymbol{u}\left(t_{n}\right)\right\|_{k+1, \Sigma}+\tau^{\frac{1}{2}}\left\|\partial_{t} \boldsymbol{u}\right\|_{L^{2}\left(t_{n-1}, t_{n} ; H^{1}(\Sigma)\right)}\right) \\
\left\|\boldsymbol{z}_{h}^{n}-\boldsymbol{z}_{h}^{n-1}\right\|_{\mathrm{e}} \lesssim & \left(\beta^{\mathrm{e}}\right)^{\frac{1}{2}} \tau^{\frac{1}{2}} h^{k}\left\|\partial_{t} \boldsymbol{u}\right\|_{L^{2}\left(t_{n-1}, t_{n} ; H^{k+1}(\Sigma)\right)} \\
& +\left(\beta^{\mathrm{e}}\right)^{\frac{1}{2}} \tau^{\frac{3}{2}}\left\|\partial_{t t} \boldsymbol{u}\right\|_{L^{2}\left(t_{n-1}, t_{n} ; H^{1}(\Sigma)\right)}
\end{aligned}
$$

which can be inferred from (32) and standard Taylor expansions.

Taking $\left(\boldsymbol{v}_{h}, q_{h}\right)=\tau\left(\boldsymbol{\theta}_{h}^{n}, y_{h}^{n}\right)$ in (70), and using (71)-(72), yields the following energy equation for the discrete errors:

$$
\begin{aligned}
& \frac{\rho^{\mathrm{f}}}{2}\left(\tau \partial_{\tau}\left\|\boldsymbol{\theta}_{h}^{n}\right\|_{0, \Omega}^{2}+\tau^{2}\left\|\partial_{\tau} \boldsymbol{\theta}_{h}^{n}\right\|_{0, \Omega}^{2}\right)+2 \mu \tau\left\|\varepsilon\left(\boldsymbol{\theta}_{h}^{n}\right)\right\|_{0, \Omega}^{2}+\tau\left|y_{h}^{n}\right|_{s_{h}}^{2} \\
& +\frac{\rho^{\mathrm{s}} \epsilon}{2}\left(\tau \partial_{\tau}\left\|\dot{\boldsymbol{\xi}}_{h}^{n}\right\|_{0, \Sigma}^{2}+\tau^{2}\left\|\partial_{\tau} \dot{\boldsymbol{\xi}}_{h}^{n}\right\|_{0, \Sigma}^{2}\right)+\frac{1}{2}\left(\tau \partial_{\tau}\left\|\boldsymbol{\xi}_{h}^{n}\right\|_{\mathrm{e}}^{2}+\tau^{2}\left\|\partial_{\tau} \boldsymbol{\xi}_{h}^{n}\right\|_{\mathrm{e}}^{2}\right) \\
& +\tau\left\|\dot{\boldsymbol{\xi}}_{h}^{n}\right\|_{\mathrm{v}}^{2}+\underbrace{\tau^{2}\left(\partial_{\tau} \dot{\boldsymbol{\xi}}_{h}^{n}, \boldsymbol{L}_{h}^{\mathrm{e}}\left(\boldsymbol{\xi}_{h}^{n}-\boldsymbol{\xi}_{h}^{\star}\right)+\boldsymbol{L}_{h}^{\mathrm{v}}\left(\dot{\boldsymbol{\xi}}_{h}^{n}-\dot{\boldsymbol{\xi}}_{h}^{\star}\right)\right)_{\Sigma}}_{T_{5}} \\
& +\underbrace{\frac{\tau^{2}}{\rho^{\mathrm{s} \epsilon}}\left(\boldsymbol{L}_{h}^{\mathrm{e}} \boldsymbol{\xi}_{h}^{n}+\boldsymbol{L}_{h}^{\mathrm{v}} \dot{\boldsymbol{\xi}}_{h}^{n}, \boldsymbol{L}_{h}^{\mathrm{e}}\left(\boldsymbol{\xi}_{h}^{n}-\boldsymbol{\xi}_{h}^{\star}\right)+\boldsymbol{L}_{h}^{\mathrm{v}}\left(\dot{\boldsymbol{\xi}}_{h}^{n}-\dot{\boldsymbol{\xi}}_{h}^{\star}\right)\right)_{\Sigma}}_{T_{6}} \\
& =T_{1}\left(\tau \boldsymbol{\theta}_{h}^{n}\right)+T_{2}\left(\tau \boldsymbol{\theta}_{h}^{n}\right)+T_{3}\left(\tau \boldsymbol{\theta}_{h}^{n}, \tau y_{h}^{n}\right)-\underbrace{\tau a^{\mathrm{e}}\left(\boldsymbol{\xi}_{h}^{n}, \boldsymbol{z}_{h}^{n}\right)}_{T_{4}} \\
& +\underbrace{\tau^{2}\left(\partial_{\tau} \dot{\boldsymbol{\xi}}_{h}^{n}, \boldsymbol{L}_{h}^{\mathrm{e}}\left(\boldsymbol{d}\left(t_{n}\right)-\boldsymbol{d}^{\star}\right)+\boldsymbol{L}_{h}^{\mathrm{v}}\left(\dot{\boldsymbol{d}}\left(t_{n}\right)-\dot{\boldsymbol{d}}^{\star}\right)\right)_{\Sigma}}_{T_{7}} \\
& +\underbrace{\frac{\tau^{2}}{\rho^{\mathrm{s} \epsilon}}\left(\boldsymbol{L}_{h}^{\mathrm{e}} \boldsymbol{\xi}_{h}^{n}+\boldsymbol{L}_{h}^{\mathrm{v}} \dot{\boldsymbol{\xi}}_{h}^{n}, \boldsymbol{L}_{h}^{\mathrm{e}}\left(\boldsymbol{d}\left(t_{n}\right)-\boldsymbol{d}^{\star}\right)+\boldsymbol{L}_{h}^{\mathrm{v}}\left(\dot{\boldsymbol{d}}_{\left(t_{n}\right)} \dot{\boldsymbol{d}}^{\star}\right)\right)_{\Sigma}}_{T_{8}}
\end{aligned}
$$

for $n \geq r^{\star}+1$. The terms $T_{1}, \ldots, T_{4}$ are estimated as in [21, Section 5], using (32)-(34), (73) $\mathrm{RR} \mathrm{n}^{\circ} 8224$ 
and Taylor expansions. We simply recall the corresponding bound

$$
\begin{aligned}
\sum_{i=1}^{4} T_{i} \lesssim & \frac{\left(\rho^{\mathrm{f}} \tau\right)^{2}}{\varepsilon_{1} \mu}\left\|\partial_{t t} \boldsymbol{u}\right\|_{L^{2}\left(t_{n-1}, t_{n} ; L^{2}(\Omega)\right)}^{2}+\frac{\left(\rho^{\mathrm{f}} c_{\mu}\right)^{2}}{\varepsilon_{1}} h^{2 k+2}\left\|\partial_{t} \boldsymbol{u}\right\|_{L^{2}\left(t_{n-1}, t_{n} ; H^{k+1}(\Omega)\right)}^{2} \\
& +\left(\frac{\left(\rho^{\mathrm{f}} \tilde{c}_{\mu}\right)^{2}}{\varepsilon_{1} \mu}+\frac{\left(\rho^{\mathrm{s}} \epsilon\right)^{2}}{\varepsilon_{2} \mu}\right) h^{2 k+2}\left\|\partial_{t} \boldsymbol{u}\right\|_{L^{2}\left(t_{n-1}, t_{n} ; H^{k+1}(\Sigma)\right)}^{2} \\
& +\frac{\left(\rho^{\mathrm{s}} \epsilon \tau\right)^{2}}{\varepsilon_{2} \mu}\left\|\partial_{t t} \boldsymbol{u}\right\|_{L^{2}\left(t_{n-1}, t_{n} ; L^{2}(\Sigma)\right)}^{2}+\tau^{2} \beta^{\mathrm{e}} T\left\|\partial_{t} \boldsymbol{u}\right\|_{L^{2}\left(t_{n-1}, t_{n} ; H^{1}(\Sigma)\right)}^{2} \\
& +\tau\left(\frac{\mu h^{2 k}}{\varepsilon_{3}}\left\|\boldsymbol{u}\left(t_{n}\right)\right\|_{k+1, \Omega}^{2}+\frac{h^{2 \tilde{l}}}{\varepsilon_{3} \mu}\left\|p\left(t_{n}\right)\right\|_{\tilde{l}, \Omega}^{2}+h^{2 k} \beta^{\mathrm{e}} T\left\|\boldsymbol{u}\left(t_{n}\right)\right\|_{k+1, \Sigma}^{2}\right) \\
& +\tau\left(\varepsilon_{1}+\varepsilon_{2}+\varepsilon_{3}\right)\left\|\boldsymbol{\theta}_{h}^{n}\right\|_{\boldsymbol{V}}^{2}+\tau \varepsilon_{3}\left|y_{h}^{n}\right|_{s_{h}}^{2}+\frac{\tau}{T}\left\|\boldsymbol{\xi}_{h}^{n}\right\|_{\mathrm{e}}^{2}
\end{aligned}
$$

where $\varepsilon_{i}, i=1, \ldots, 3$, are free positive parameters. In $(75)$, the terms involving $\left\|\boldsymbol{\theta}_{h}^{n}\right\|_{\boldsymbol{V}}^{2}$ and $\left|y_{h}^{n}\right|_{s_{h}}^{2}$ can be absorbed by the left-hand side of (74), by choosing $\varepsilon_{i}$ sufficiency small, while the last term is controlled via Gronwall's Lemma.

As regards the remaining terms $T_{5}, \ldots, T_{8}$ in (74), we proceed by treating each extrapolation separately.

(i) Without extrapolation $\left(r^{\star}=0\right)$. Terms $T_{5}$ and $T_{6}$ are bounded as terms $T_{1}$ and $T_{2}$ in $A$, which yields

$$
T_{5}+T_{6} \geq-\frac{\rho^{\mathrm{s}} \epsilon}{3}\left\|\dot{\boldsymbol{\xi}}_{h}^{n}-\dot{\boldsymbol{\xi}}_{h}^{n-1}\right\|_{0, \Sigma}^{2}+\frac{\tau^{2}}{4 \rho^{\mathrm{s}} \epsilon}\left\|\boldsymbol{L}_{h}^{\mathrm{e}} \boldsymbol{\xi}_{h}^{n}+\boldsymbol{L}_{h}^{\mathrm{v}} \dot{\boldsymbol{\xi}}_{h}^{n}\right\|_{0, \Sigma}^{2}
$$

For the remaining terms, using Young's inequality and (68), we have

$$
\begin{aligned}
T_{7}+T_{8} \leq & \frac{\rho^{\mathrm{s}} \epsilon}{8}\left\|\dot{\boldsymbol{\xi}}_{h}^{n}-\dot{\boldsymbol{\xi}}_{h}^{n-1}\right\|_{0, \Sigma}^{2}+\frac{\tau^{2}}{8 \rho^{\mathrm{s}} \epsilon}\left\|\boldsymbol{L}_{h}^{\mathrm{e}} \boldsymbol{\xi}_{h}^{n}+\boldsymbol{L}_{h}^{\mathrm{v}} \dot{\boldsymbol{\xi}}_{h}^{n}\right\|_{0, \Sigma}^{2} \\
& +\frac{4 \tau^{2}}{\rho^{\mathrm{s}} \epsilon}\left\|\boldsymbol{L}^{\mathrm{e}} \boldsymbol{d}\left(t_{n}\right)+\boldsymbol{L}^{\mathrm{v}} \dot{\boldsymbol{d}}\left(t_{n}\right)\right\|_{0, \Sigma}^{2}
\end{aligned}
$$

The estimate (64) then follows by inserting (75), (76) and (77) into (74), using Korn's inequality, summing over $m=1, \ldots, n$, and applying Gronwall's lemma.

(ii) First-order extrapolation $\left(r^{\star}=1\right)$. Terms $T_{6}$ is treated as term $T_{2}$ in A, yielding

$$
T_{6}=\frac{\tau^{2}}{2 \rho^{\mathrm{s}} \epsilon}\left(\tau \partial_{\tau}\left\|\boldsymbol{L}_{h}^{\mathrm{e}} \boldsymbol{\xi}_{h}^{n}+\boldsymbol{L}_{h}^{\mathrm{v}} \dot{\boldsymbol{\xi}}_{h}^{n}\right\|_{0, \Sigma}^{2}+\tau^{2}\left\|\partial_{\tau}\left(\boldsymbol{L}_{h}^{\mathrm{e}} \boldsymbol{\xi}_{h}^{n}+\boldsymbol{L}_{h}^{\mathrm{v}} \dot{\boldsymbol{\xi}}_{h}^{n}\right)\right\|_{0, \Sigma}^{2}\right)
$$

For term $T_{5}$, we have

$$
T_{5}=\frac{\tau^{2}}{2}\left(\tau \partial_{\tau}\left\|\dot{\boldsymbol{\xi}}_{h}^{n}\right\|_{\mathrm{e}}^{2}+\left\|\dot{\boldsymbol{\xi}}_{h}^{n}-\dot{\boldsymbol{\xi}}_{h}^{n-1}\right\|_{\mathrm{e}}^{2}\right)+\tau\left\|\dot{\boldsymbol{\xi}}_{h}^{n}-\dot{\boldsymbol{\xi}}_{h}^{n-1}\right\|_{\mathrm{v}}^{2}+\tau^{2} a^{\mathrm{e}}\left(\dot{\boldsymbol{\xi}}_{h}^{n}-\dot{\boldsymbol{\xi}}_{h}^{n-1}, \boldsymbol{z}_{h}^{n}\right),
$$

where the last contribution is due to the modified displacement-velocity relation (72). Thus, using (73), we get

$$
\begin{aligned}
T_{5} \gtrsim & \frac{\tau^{2}}{2}\left(\left\|\dot{\boldsymbol{\xi}}_{h}^{n}\right\|_{\mathrm{e}}^{2}-\left\|\dot{\boldsymbol{\xi}}_{h}^{n-1}\right\|_{\mathrm{e}}^{2}\right)+\tau\left\|\dot{\boldsymbol{\xi}}_{h}^{n}-\dot{\boldsymbol{\xi}}_{h}^{n-1}\right\|_{\mathrm{v}}^{2}-\beta^{\mathrm{e}} h^{2 k} \tau^{2}\left\|\boldsymbol{u}\left(t_{n}\right)\right\|_{k+1, \Sigma}^{2} \\
& -\beta^{\mathrm{e}} \tau^{3}\left\|\partial_{t} \boldsymbol{u}\right\|_{L^{2}\left(t_{n-1}, t_{n} ; H^{1}(\Sigma)\right)}^{2}
\end{aligned}
$$


For the terms $T_{7}$ and $T_{8}$, using Taylor expansions and (68), we have

$$
\begin{aligned}
T_{7}+T_{8} \leq & \frac{\rho^{\mathrm{s}} \epsilon}{2 T}\left(\left\|\dot{\boldsymbol{\xi}}_{h}^{n}\right\|_{0, \Sigma}^{2}+\left\|\dot{\boldsymbol{\xi}}_{h}^{n-1}\right\|_{0, \Sigma}^{2}\right)+\frac{3 T \tau^{2}}{2 \rho^{\mathrm{s}} \epsilon}\left\|\boldsymbol{L}^{\mathrm{e}} \partial_{t} \boldsymbol{d}+\boldsymbol{L}^{\mathrm{v}} \partial_{t} \dot{\boldsymbol{d}}\right\|_{L^{2}\left(t_{n-1}, t_{n} ; L^{2}(\Sigma)\right)}^{2} \\
& +\frac{\tau^{3}}{2 \rho^{\mathrm{s}} \epsilon T}\left\|\boldsymbol{L}_{h}^{\mathrm{e}} \boldsymbol{\xi}_{h}^{n}+\boldsymbol{L}_{h}^{\mathrm{v}} \dot{\boldsymbol{\xi}}_{h}^{n}\right\|_{0, \Sigma}^{2}
\end{aligned}
$$

where last term can be treated via Gronwall's Lemma, using the control provided by (78).

In summary, the estimate (65) follows by inserting the above bounds into (74), using Korn's inequality, summing over $m=2, \ldots, n$, and applying Gronwall's Lemma. The part of the discrete error corresponding to the initialization step is bounded from (64) with $n=1$ and $T=\tau$.

(iii) Second-order extrapolation $\left(r^{\star}=2\right)$. Terms $T_{5}$ and $T_{6}$ are treated as terms $T_{1}$ and $T_{2}$ in A, apart from the additional contribution of the modified displacement-velocity relation (72). We have

$$
\begin{aligned}
T_{5}= & \tau^{2}\left\|\dot{\boldsymbol{\xi}}_{h}^{n}-\dot{\boldsymbol{\xi}}_{h}^{n-1}\right\|_{\mathrm{e}}^{2}+\frac{\tau}{2}\left(\tau \partial_{\tau}\left\|\dot{\boldsymbol{\xi}}_{h}^{n}-\dot{\boldsymbol{\xi}}_{h}^{n-1}\right\|_{\mathrm{v}}^{2}+\left\|\dot{\boldsymbol{\xi}}_{h}^{n}-2 \dot{\boldsymbol{\xi}}_{h}^{n-1}+\dot{\boldsymbol{\xi}}_{h}^{n-2}\right\|_{\mathrm{v}}^{2}\right) \\
& +\underbrace{\tau^{2} a^{\mathrm{e}}\left(\dot{\boldsymbol{\xi}}_{h}^{n}-\dot{\boldsymbol{\xi}}_{h}^{n-1}, \boldsymbol{z}_{h}^{n}-\boldsymbol{z}_{h}^{n-1}\right)}_{T_{5,1}},
\end{aligned}
$$

where the last term is bounded, using (73), as follows

$$
\begin{aligned}
T_{5,1} \gtrsim & -\frac{\tau^{2}}{2}\left\|\dot{\boldsymbol{\xi}}_{h}^{n}-\dot{\boldsymbol{\xi}}_{h}^{n-1}\right\|_{\mathrm{e}}^{2} \\
& -\frac{\beta^{\mathrm{e}} \tau^{3}}{2}\left(h^{2 k}\left\|\partial_{t} \boldsymbol{u}\right\|_{L^{2}\left(t_{n-1}, t_{n} ; H^{k+1}(\Sigma)\right)}^{2}+\tau^{2}\left\|\partial_{t t} \boldsymbol{u}\right\|_{L^{2}\left(t_{n-1}, t_{n} ; H^{1}(\Sigma)\right)}^{2}\right) .
\end{aligned}
$$

For $T_{6}$, using (72), we have

$$
\begin{aligned}
T_{6} \geq & \frac{\alpha \tau^{3}}{2 \rho^{\mathrm{s}} \epsilon}\left(\tau \partial_{\tau}\left\|\dot{\boldsymbol{\xi}}_{h}^{n}\right\|_{\mathrm{e}}^{2}+\left\|\dot{\boldsymbol{\xi}}_{h}^{n}-\dot{\boldsymbol{\xi}}_{h}^{n-1}\right\|_{\mathrm{e}}^{2}\right) \\
& +\frac{\beta \tau^{3}}{2 \rho^{\mathrm{s}} \epsilon}\left(\tau \partial_{\tau}\left\|\boldsymbol{L}_{h}^{\mathrm{e}} \dot{\boldsymbol{\xi}}_{h}^{n}\right\|_{0, \Sigma}^{2}+\left\|\boldsymbol{L}_{h}^{\mathrm{e}}\left(\dot{\boldsymbol{\xi}}_{h}^{n}-\dot{\boldsymbol{\xi}}_{h}^{n-1}\right)\right\|_{0, \Sigma}^{2}\right) \\
& -\tau \frac{\gamma}{2}\left\|\boldsymbol{\xi}_{h}^{n}\right\|_{\mathrm{e}}^{2}-\tau \delta\left\|\dot{\boldsymbol{\xi}}_{h}^{n}\right\|_{\mathrm{v}}^{2}-\frac{\rho^{\mathrm{s}} \epsilon}{4}\left\|\dot{\boldsymbol{\xi}}_{h}^{n}-\dot{\boldsymbol{\xi}}_{h}^{n-1}\right\|_{0, \Sigma}^{2} \\
& -\frac{\tau}{4}\left\|\dot{\boldsymbol{\xi}}_{h}^{n}-2 \dot{\boldsymbol{\xi}}_{h}^{n-1}+\dot{\boldsymbol{\xi}}_{h}^{n-2}\right\|_{\mathrm{v}}^{2} \underbrace{-\frac{\tau^{3}}{\rho^{\mathrm{s}} \epsilon} a^{\mathrm{e}}\left(\boldsymbol{L}_{h}^{\mathrm{e}} \boldsymbol{\xi}_{h}^{n}+\boldsymbol{L}_{h}^{\mathrm{v}} \dot{\boldsymbol{\xi}}_{h}^{n}, \boldsymbol{z}_{h}^{n}-\boldsymbol{z}_{h}^{n-1}\right)}_{T_{6,1}} .
\end{aligned}
$$

The last term in this bounded through (3), (48), (50) and (51) as follows

$\mathrm{RR} \mathrm{n}^{\circ} 8224$

$$
\begin{aligned}
T_{6,1} \geq & -\frac{\tau^{5}}{2 T\left(\rho^{\mathrm{s}} \epsilon\right)^{2}}\left\|\boldsymbol{L}_{h}^{\mathrm{e}} \boldsymbol{\xi}_{h}^{n}+\boldsymbol{L}_{h}^{\mathrm{v}} \dot{\boldsymbol{\xi}}_{h}^{n}\right\|_{\mathrm{e}}^{2}-\frac{\tau T}{2}\left\|\boldsymbol{z}_{h}^{n}-\boldsymbol{z}_{h}^{n-1}\right\|_{\mathrm{e}}^{2} \\
\geq & -\frac{\beta^{\mathrm{e}} C_{\mathrm{inv}}^{2} \tau^{5}}{T\left(\rho^{\mathrm{s}} \epsilon\right)^{2} h^{2}}\left(\left\|\boldsymbol{L}_{h}^{\mathrm{e}} \boldsymbol{\xi}_{h}^{n}\right\|_{0, \Sigma}^{2}+\left\|\boldsymbol{L}_{h}^{\mathrm{v}} \dot{\boldsymbol{\xi}}_{h}^{n}\right\|_{0, \Sigma}^{2}\right)-\frac{\tau T}{2}\left\|\boldsymbol{z}_{h}^{n}-\boldsymbol{z}_{h}^{n-1}\right\|_{\mathrm{e}}^{2} \\
\geq & -\frac{\beta^{\mathrm{e}} C_{\mathrm{inv}}^{2} \tau^{5}}{T\left(\rho^{\mathrm{s}} \epsilon\right)^{2} h^{2}}\left[\frac{\beta^{\mathrm{e}} C_{\mathrm{inv}}^{2}}{h^{2}}\left\|\boldsymbol{\xi}_{h}^{n}\right\|_{\mathrm{e}}^{2}+\left(\alpha \rho^{\mathrm{s} \epsilon}+\beta \frac{\beta^{\mathrm{e}} C_{\mathrm{inv}}^{2}}{h^{2}}\right)\left\|\dot{\boldsymbol{\xi}}_{h}^{n}\right\|_{\mathrm{v}}^{2}\right] \\
& -\frac{\tau T}{2}\left\|\boldsymbol{z}_{h}^{n}-\boldsymbol{z}_{h}^{n-1}\right\|_{\mathrm{e}}^{2} \\
\geq & -\frac{\tau^{5}\left(\omega^{\mathrm{e}}\right)^{4}}{T h^{4}}\left\|\boldsymbol{\xi}_{h}^{n}\right\|_{\mathrm{e}}^{2}-\frac{\tau^{5}\left(\omega^{\mathrm{e}}\right)^{2}}{T h^{2}}\left(\alpha+\frac{\beta\left(\omega^{\mathrm{e}}\right)^{2}}{h^{2}}\right)\left\|\dot{\boldsymbol{\xi}}_{h}^{n}\right\|_{\mathrm{v}}^{2}-\frac{\tau T}{2}\left\|\boldsymbol{z}_{h}^{n}-\boldsymbol{z}_{h}^{n-1}\right\|_{\mathrm{e}}^{2},
\end{aligned}
$$


where we have used the notation $\omega^{\mathrm{e}} \stackrel{\text { def }}{=} C_{\mathrm{inv}} \sqrt{\beta^{\mathrm{e}} /\left(\rho^{\mathrm{s}} \epsilon\right)}$. Hence, using (29) and (73), we finally infer that

$$
\begin{aligned}
T_{6,1} \gtrsim & -\tau^{2} \beta^{\mathrm{e}} T\left(h^{2 k}\left\|\partial_{t} \boldsymbol{u}\right\|_{L^{2}\left(t_{n-1}, t_{n} ; H^{k+1}(\Sigma)\right)}^{2}+\tau^{2}\left\|\partial_{t t} \boldsymbol{u}\right\|_{L^{2}\left(t_{n-1}, t_{n} ; H^{1}(\Sigma)\right)}^{2}\right) \\
& -\frac{\tau}{T}\left(\frac{\gamma \tau}{2}\right)^{\frac{2}{3}}\left\|\boldsymbol{\xi}_{h}^{n}\right\|_{\mathrm{e}}^{2}-\frac{\tau^{3} \gamma}{4 T}\left\|\dot{\boldsymbol{\xi}}_{h}^{n}\right\|_{\mathrm{v}}^{2} .
\end{aligned}
$$

The first of the last two terms is controlled via Gronwall's Lemma, while the second can be absorbed by the left-hand side of (74).

We conclude the proof with the estimation of the remaining terms in the right-hand side of (74). For $T_{7}$, using a Taylor expansion, we simply have

$$
T_{7} \leq \tau \frac{\rho^{\mathrm{s}} \epsilon}{2 T}\left(\left\|\dot{\boldsymbol{\xi}}_{h}^{n}\right\|_{0, \Sigma}^{2}+\left\|\dot{\boldsymbol{\xi}}_{h}^{n-1}\right\|_{0, \Sigma}^{2}\right)+\frac{T \tau^{4}}{2 \rho^{\mathrm{s}} \epsilon}\left\|\boldsymbol{L}^{\mathrm{e}} \partial_{t t} \boldsymbol{d}+\boldsymbol{L}^{\mathrm{v}} \partial_{t t} \dot{\boldsymbol{d}}\right\|_{L^{2}\left(t_{n-1}, t_{n} ; L^{2}(\Sigma)\right)}^{2}
$$

where the first term can be controlled via Gronwall's Lemma. At last, for T, using a Taylor expansion and proceeding similarly to (79), there follows

$$
\begin{aligned}
T_{8} \leq & \frac{\tau^{3}}{\rho^{\mathrm{s}} \epsilon T}\left(\left\|\boldsymbol{L}_{h}^{\mathrm{e}} \boldsymbol{\xi}_{h}^{n}\right\|_{0, \Sigma}^{2}+\left\|\boldsymbol{L}_{h}^{\mathrm{v}} \dot{\boldsymbol{\xi}}_{h}^{n}\right\|_{0, \Sigma}^{2}\right)+\frac{T \tau^{4}}{2 \rho^{\mathrm{s}} \epsilon}\left\|\boldsymbol{L}^{\mathrm{e}} \partial_{t t} \boldsymbol{d}+\boldsymbol{L}^{\mathrm{v}} \partial_{t t} \dot{\boldsymbol{d}}\right\|_{L^{2}\left(t_{n-1}, t_{n} ; L^{2}(\Sigma)\right)}^{2} \\
\leq & \frac{\tau^{3}\left(\omega^{\mathrm{e}}\right)^{2}}{T h^{2}}\left\|\boldsymbol{\xi}_{h}^{n}\right\|_{\mathrm{e}}^{2}+\frac{\tau^{3}}{T}\left(\alpha+\frac{\beta\left(\omega^{\mathrm{e}}\right)^{2}}{h^{2}}\right)\left\|\dot{\boldsymbol{\xi}}_{h}^{n}\right\|_{\mathrm{v}}^{2} \\
& +\frac{T \tau^{4}}{2 \rho^{\mathrm{s}} \epsilon}\left\|\boldsymbol{L}^{\mathrm{e}} \partial_{t t} \boldsymbol{d}+\boldsymbol{L}^{\mathrm{v}} \partial_{t t} \dot{\boldsymbol{d}}\right\|_{L^{2}\left(t^{n-1}, t^{n} ; L^{2}(\Sigma)\right)}^{2} .
\end{aligned}
$$

Hence, from (29), we infer that

$$
T_{8} \leq \frac{\tau}{T}\left(\frac{\tau \gamma}{2}\right)^{\frac{1}{3}}\left\|\boldsymbol{\xi}_{h}^{n}\right\|_{\mathrm{e}}^{2}+\frac{\tau^{2} \delta^{\frac{1}{2}}}{T}\left\|\dot{\boldsymbol{\xi}}_{h}^{n}\right\|_{\mathrm{v}}^{2}+\frac{T \tau^{4}}{2 \rho^{\mathrm{s}} \epsilon}\left\|\boldsymbol{L}^{\mathrm{e}} \partial_{t t} \boldsymbol{d}+\boldsymbol{L}^{\mathrm{v}} \partial_{t t} \dot{\boldsymbol{d}}\right\|_{L^{2}\left(t^{n-1}, t^{n} ; L^{2}(\Sigma)\right)}^{2}
$$

where the first two terms are controlled as in (80), via Gronwall's Lemma and the left-hand side of (74), respectively.

In summary, the estimate (66) follows by inserting the above bounds into (74), using Korn's inequality, summing over $m=3, \ldots, n$, and applying Gronwall's Lemma. The remaining terms, corresponding to the initialization step, are bounded via (65) with $n=2$ and $T=2 \tau$.

\section{References}

[1] M. Astorino, F. Chouly, and M. A. Fernández. Robin based semi-implicit coupling in fluidstructure interaction: Stability analysis and numerics. SIAM J. Sci. Comput., 31(6):40414065, 2009.

[2] S. Badia, F. Nobile, and C. Vergara. Fluid-structure partitioned procedures based on Robin transmission conditions. J. Comp. Phys., 227:7027-7051, 2008.

[3] Y. Bazilevs, V.M. Calo, T.J.R. Hughes, and Y. Zhang. Isogeometric fluid-structure interaction: theory, algorithms, and computations. Comput. Mech., 43(1):3-37, 2008.

[4] M. Bischoff, K.-U. Bletzinger, W.A. Wall, and E. Ramm. Models and Finite Elements for Thin-Walled Structures, chapter 3. John Wiley \& Sons, Ltd, 2004. 
[5] D. Boffi, L. Gastaldi, and L. Heltai. Numerical stability of the finite element immersed boundary method. Math. Models Methods Appl. Sci., 17(10):1479-1505, 2007.

[6] F. Brezzi and J. Pitkäranta. On the stabilization of finite element approximations of the Stokes equations. volume 10 of Notes Numer. Fluid Mech., pages 11-19. Vieweg, 1984.

[7] M. Bukac, S. Canic, R. Glowinski, J. Tambaca, and A. Quaini. Fluid-structure interaction in blood flow capturing non-zero longitudinal structure displacement. J. Comp. Phys., $235: 515-541,2013$

[8] E. Burman and M.A. Fernández. Stabilized explicit coupling for fluid-structure interaction using Nitsche's method. C. R. Math. Acad. Sci. Paris, 345(8):467-472, 2007.

[9] E. Burman and M.A. Fernández. Galerkin finite element methods with symmetric pressure stabilization for the transient Stokes equations: stability and convergence analysis. SIAM J. Numer. Anal., 47(1):409-439, 2008.

[10] E. Burman and M.A. Fernández. Stabilization of explicit coupling in fluid-structure interaction involving fluid incompressibility. Comput. Methods Appl. Mech. Engrg., 198(5-8):766$784,2009$.

[11] S. Čanić, J. Tambača, G. Guidoboni, A. Mikelić, C.J. Hartley, and D. Rosenstrauch. Modeling viscoelastic behavior of arterial walls and their interaction with pulsatile blood flow. SIAM J. Appl. Math., 67(1):164-193, 2006.

[12] P. Causin, J.-F. Gerbeau, and F. Nobile. Added-mass effect in the design of partitioned algorithms for fluid-structure problems. Comput. Methods Appl. Mech. Engrg., 194(4244):4506-4527, 2005.

[13] D. Chapelle and K.J. Bathe. The Finite Element Analysis of Shells - Fundamentals. Springer, 2011.

[14] D. Chapelle and A. Ferent. Modeling of the inclusion of a reinforcing sheet within a 3D medium. Math. Models Methods Appl. Sci., 13(4):573-595, 2003.

[15] P. Crosetto, S. Deparis, G. Fourestey, and A. Quarteroni. Parallel algorithms for fluidstructure interaction problems in haemodynamics. SIAM J. Sci. Comput., 33(4):1598-1622, 2011 .

[16] S. Deparis, M. Discacciati, G. Fourestey, and A. Quarteroni. Fluid-structure algorithms based on Steklov-Poincaré operators. Comput. Methods Appl. Mech. Engrg., 195(4143):5797-5812, 2006.

[17] W. Dettmer and D. Perić. A computational framework for fluid-rigid body interaction: finite element formulation and applications. Comput. Methods Appl. Mech. Engrg., 195(1316):1633-1666, 2006.

[18] M. Eswaran, U.K. Saha, and D. Maity. Effect of baffles on a partially filled cubic tank: Numerical simulation and experimental validation. Computers \& Structures, 87(3-4):198$205,2009$.

[19] M.A. Fernández. Coupling schemes for incompressible fluid-structure interaction: implicit, semi-implicit and explicit. S $\overrightarrow{\mathrm{e}} M A$ J., (55):59-108, 2011. 
[20] M.A. Fernández. Incremental displacement-correction schemes for the explicit coupling of a thin structure with an incompressible fluid. C. R. Math. Acad. Sci. Paris, 349(7-8):473-477, 2011.

[21] M.A. Fernández. Incremental displacement-correction schemes for incompressible fluidstructure interaction: stability and convergence analysis. Numer. Math., 123(1):21-65, 2013.

[22] M.A. Fernández, J.F. Gerbeau, and C. Grandmont. A projection semi-implicit scheme for the coupling of an elastic structure with an incompressible fluid. Int. J. Num. Meth. Engrg., 69(4):794-821, 2007.

[23] M.A. Fernández and M. Moubachir. A Newton method using exact Jacobians for solving fluid-structure coupling. Comp. \& Struct., 83:127-142, 2005.

[24] M.A. Fernández and J. Mullaert. Displacement-velocity correction schemes for incompressible fluid-structure interaction. C. R. Math. Acad. Sci. Paris, 349(17-18):1011-1015, 2011.

[25] M.A. Fernández, J. Mullaert, and M. Vidrascu. Generalized Robin-Neumann explicit coupling schemes for incompressible fluid-structure interaction.

Part I: Stability analysis and numerics. In preparation, 2013.

[26] L. Formaggia, J.-F. Gerbeau, F. Nobile, and A. Quarteroni. On the coupling of 3D and 1D Navier-Stokes equations for flow problems in compliant vessels. Comp. Meth. Appl. Mech. Engrg., 191(6-7):561-582, 2001.

[27] L. Formaggia, A. Quarteroni, and A. Veneziani, editors. Cardiovascular Mathematics. Modeling and simulation of the circulatory system, volume 1 of Modeling, Simulation and Applications. Springer, 2009.

[28] C. Förster, W.A. Wall, and E. Ramm. Artificial added mass instabilities in sequential staggered coupling of nonlinear structures and incompressible viscous flows. Comput. Methods Appl. Mech. Engrg., 196(7):1278-1293, 2007.

[29] M. Gee, E. Ramm, and W.-A. Wal. Parallel multilevel solution of nonlinear shell structures. Comput. Methods Appl. Mech. Engrg., 194(21-24):2513-2533, 2005.

[30] M.W. Gee, U. Küttler, and W. Wall. Truly monolithic algebraic multigrid for fluid-structure interaction. Int. J. Numer. Meth. Engng., 85(8):987-1016, 2011.

[31] J.-F. Gerbeau and M. Vidrascu. A quasi-Newton algorithm based on a reduced model for fluid-structure interactions problems in blood flows. Math. Model. Num. Anal., 37(4):631$648,2003$.

[32] G. Guidoboni, R. Glowinski, N. Cavallini, and S. Canic. Stable loosely-coupled-type algorithm for fluid-structure interaction in blood flow. J. Comp. Phys., 228(18):6916-6937, 2009.

[33] P. Hansbo. Nitsche's method for interface problems in computational mechanics. GAMMMitt., 28(2):183-206, 2005.

[34] M. Heil. An efficient solver for the fully coupled solution of large-displacement fluid-structure interaction problems. Comput. Methods Appl. Mech. Engrg., 193(1-2):1-23, 2004.

[35] M. Heil and A.L. Hazel. Fluid-structure interaction in internal physiological flows. Annu. Rev. Fluid Mech., 43:141-162, 2011. 
[36] J.G. Heywood and R. Rannacher. Finite-element approximation of the nonstationary NavierStokes problem. IV. Error analysis for second-order time discretization. SIAM J. Numer. Anal., 27(2):353-384, 1990.

[37] T.J.R. Hughes. The finite element method. Prentice Hall, 1987.

[38] P. Kalita and R. Schaefer. Mechanical models of artery walls. Arch. Comput. Methods Eng., 15(1):1-36, 2008.

[39] U. Küttler and W.A. Wall. Fixed-point fluid-structure interaction solvers with dynamic relaxation. Comp. Mech., 43(1):61-72, 2008.

[40] U. Küttler, C. Förster, and W.A. Wall. A solution for the incompressibility dilemma in partitioned fluid-structure interaction with pure Dirichlet fluid domains. Comput. Mech., 38:417-429, 2006.

[41] P. Le Tallec and J. Mouro. Fluid structure interaction with large structural displacements. Comput. Meth. Appl. Mech. Engrg., 190:3039-3067, 2001.

[42] M. Lombardi, N. Parolini, A. Quarteroni, and G. Rozza. Numerical simulation of sailing boats: Dynamics, FSI, and shape optimization. In G. Buttazzo and A. Frediani, editors, Variational Analysis and Aerospace Engineering: Mathematical Challenges for Aerospace Design, Springer Optimization and Its Applications, pages 339-377. Springer, 2012.

[43] M. Lukáčová, G. Rusnáková, and A. Hundertmark. Kinematic splitting algorithm for fluid-structure interaction in hemodynamics. Comput. Methods Appl. Mech. Engrg., 265(1):83-106, 2013.

[44] P. Moireau, N. Xiao, M. Astorino, C.A. Figueroa, D. Chapelle, C. A. Taylor, and J-F. Gerbeau. External tissue support and fluid-structure simulation in blood flows. Biomech. Model. Mechanobiol., 11:1-18, 2012.

[45] R.L. Muddle, M. Mihajlović, and M. Heil. An efficient preconditioner for monolithicallycoupled large-displacement fluid-structure interaction problems with pseudo-solid mesh updates. J. Comput. Phys., 231(21):7315-7334, 2012.

[46] J. Nitsche. Über ein Variationsprinzip zur Lösung von Dirichlet-Problemen bei Verwendung von Teilräumen, die keinen Randbedingungen unterworfen sind. Abh. Math. Sem. Univ. Hamburg, 36:9-15, 1971.

[47] F. Nobile and C. Vergara. An effective fluid-structure interaction formulation for vascular dynamics by generalized Robin conditions. SIAM J. Sci. Comput., 30(2):731-763, 2008.

[48] F. Nobile and C. Vergara. Partitioned Algorithms for Fluid-Structure Interaction Problems in Haemodynamics. Milan J. Math., 80(2):443-467, 2012.

[49] M.P. Païdoussis, S.J. Price, and E. de Langre. Fluid-structure interactions: cross-flowinduced instabilities. Cambridge University Press, 2011.

[50] C.S. Peskin. The immersed boundary method. Acta Numer., 11:479-517, 2002.

[51] O. Pironneau, F. Hecht, A. Le Hyaric, and J. Morice. Freefem++, www.freefem.org/ff++.

[52] A. Quaini and A. Quarteroni. A semi-implicit approach for fluid-structure interaction based on an algebraic fractional step method. Math. Models Methods Appl. Sci., 17(6):957-983, 2007. 
[53] A.-V. Salsac, S.R. Sparks, J.M. Chomaz, and J.C. Lasheras. Evolution of the wall shear stresses during the progressive enlargement of symmetric abdominal aortic aneurysms. $J$. Fluid Mech., 550:19-51, 2006.

[54] K. Takizawa and T.E. Tezduyar. Computational methods for parachute fluid-structure interactions. Arch. Comput. Methods Eng., 19:125-169, 2012.

[55] C. Tu and C.S. Peskin. Stability and instability in the computation of flows with moving immersed boundaries: a comparison of three methods. SIAM J. Sci. Statist. Comput., 13(6):1361-1376, 1992.

[56] D. Valdez-Jasso, H.T. Banks, M.A. Haider, D. Bia, Y. Zocalo, R.L. Armentano, and M.S. Olufsen. Viscoelastic models for passive arterial wall dynamics. Adv. Appl. Math. Mech., 1(2):151-165, 2009. 


\section{Contents}

1 Introduction $\quad 3$

2 A linear model problem $\quad 5$

3 Robin-Neumann methods $\quad 6$

3.1 Explicit coupling schemes . . . . . . . . . . . . . . . . . . . . . . . . . . .

3.2 Iterative partitioned solution of implicit coupling . . . . . . . . . . . . 9

4 Stability and convergence analysis $\quad 10$

4.1 Notation and weak formulation . . . . . . . . . . . . . . . . . . 10

4.2 Space discretization: fully discrete schemes . . . . . . . . . . . . . . . 11

4.3 Kinematic perturbation of implicit coupling . . . . . . . . . . . . . . . 12

4.4 A priori energy estimates . . . . . . . . . . . . . . . . . . . . . . . . . . . . . . . . . .

4.5 Convergence of the iterative solution procedure . . . . . . . . . . . . . . 14

4.6 A priori error estimates . . . . . . . . . . . . . . . . . . 14

5 Formulation in the non-linear case $\quad 16$

5.1 The non-linear coupled problem . . . . . . . . . . . . . . . . . 16

5.2 Explicit Robin-Neumann schemes . . . . . . . . . . . . . . . . . 17

6 Numerical experiments $\quad 18$

6.1 Convergence study in a two-dimensional test-case . . . . . . . . . . . . . . . . . 19

6.2 Pressure wave propagation in a straight tube . . . . . . . . . . . . . . . . . . . . . . . . . .

6.3 Blood flow in an abdominal aortic aneurysm . . . . . . . . . . . . . . . . . . 23

6.4 Driven cavity with flexible bottom . . . . . . . . . . . . . . . . . . . . . . . . . . . . .

6.5 Damped structural instability . . . . . . . . . . . . . . . . 26

7 Conclusion $r$

$\begin{array}{lr}\text { A Proof of Theorem } 1 & 29\end{array}$

B Proof of Theorem 2 13

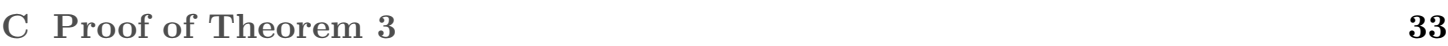




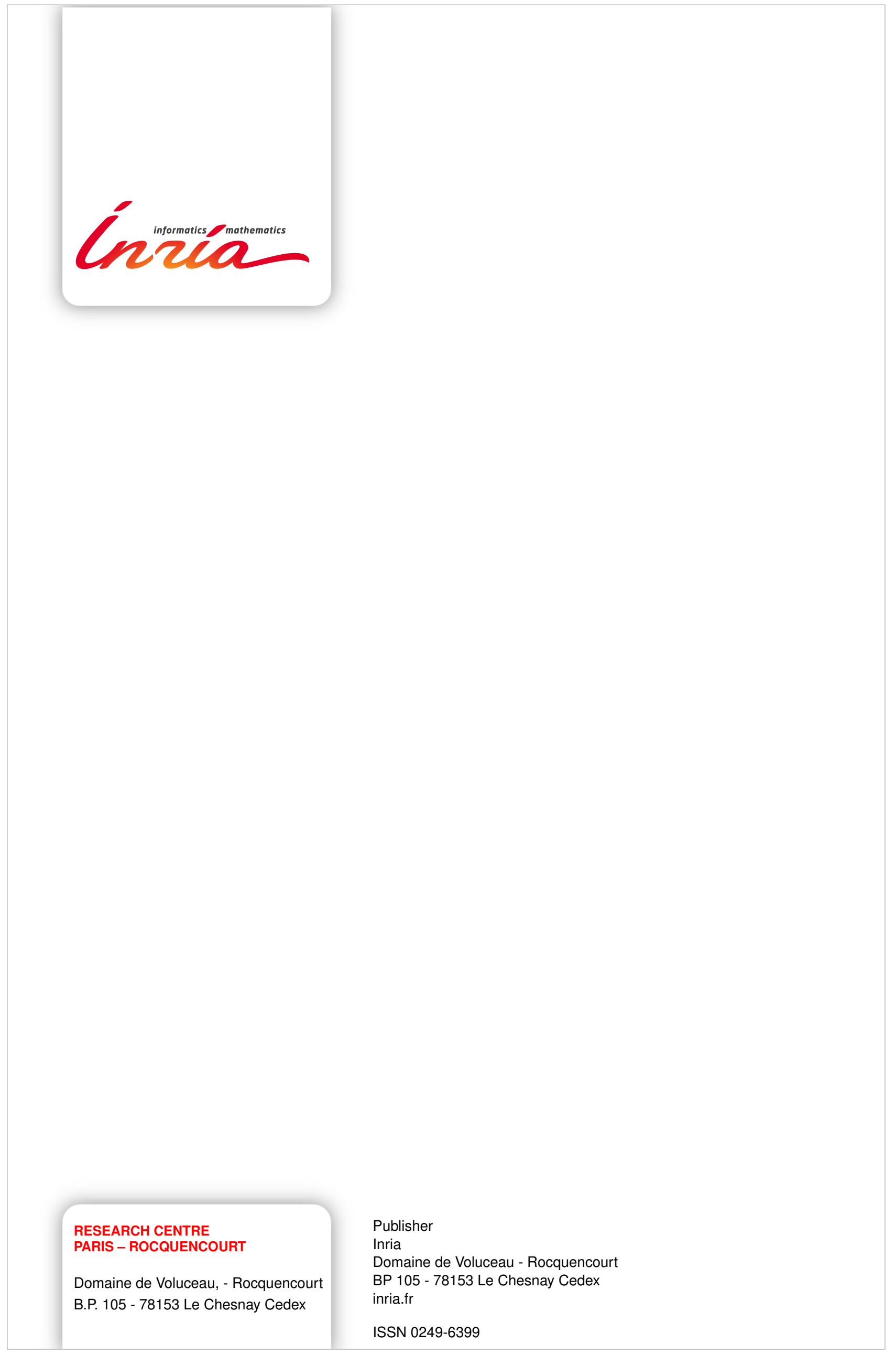

